\title{
Canonical Quantum Observables for Molecular Systems Approximated by Ab Initio Molecular Dynamics
}

\author{
Aku Kammonen, Petr Plecháč, Mattias Sandberg and \\ Anders Szepessy
}

\begin{abstract}
It is known that ab initio molecular dynamics based on the electron ground-state eigenvalue can be used to approximate quantum observables in the canonical ensemble when the temperature is low compared to the first electron eigenvalue gap. This work proves that a certain weighted average of the different ab initio dynamics, corresponding to each electron eigenvalue, approximates quantum observables for any temperature. The proof uses the semiclassical Weyl law to show that canonical quantum observables of nuclei-electron systems, based on matrix-valued Hamiltonian symbols, can be approximated by ab initio molecular dynamics with the error proportional to the electron-nuclei mass ratio. The result covers observables that depend on time correlations. A combination of the Hilbert-Schmidt inner product for quantum operators and Weyl's law shows that the error estimate holds for observables and Hamiltonian symbols that have three and five bounded derivatives, respectively, provided the electron eigenvalues are distinct for any nuclei position and the observables are in the diagonal form with respect to the electron eigenstates.
\end{abstract}

\section{Contents}

$\begin{array}{lr}\text { 1. Introduction } & 2728\end{array}$

1.1. Background 2728

1.2. Overview of Results 2730

2. The Schrödinger Equation and Gibbs Ensembles 2731

2.1. Problem Formulation and Weyl Quantization 2731

The authors are grateful to Caroline Lasser for valuable help on this work. The research was supported by Swedish Research Council 621-2014-4776 and the Swedish e-Science Research Center. The research of P.P. was supported by ARO MURI Award No. W911NF-14-024. 
2.2. Gibbs Density Operator and Its Approximation

2735

2.3. Computational Demonstration

2.3.1. Equilibrium Observables

2737

2.3.2. Time-Correlated Observables

3. Time-Correlated Observables

3.1. Solution of the Nonlinear Eigenvalue Problem

2743

3.2. Approximation in Time of Observables

2746

3.2.1. Time-Dependent Molecular Dynamics

2746

3.2.2. The Set of Allowed Observables 2747

3.3. Assumptions and Theorems

2747

3.4. Structure of the Proofs

3.4.1. Error Representation

2751

3.4.2. Estimation of Remainder Terms and Evaluation of the Trace

3.5. Remainder Terms in the Moyal Composition

4.4. Weyl Quantization Estimates: Proof of Lemmas 3.11 and 3.122765

4.4.1. The Case $A(x) \# D(x, p) \quad 2766$

4.4.2. The Case $C(x, p) \# D(x, p) \quad 2767$

4.4.3. Estimates of $\|C \# D\|_{L^{2}}$ and $\|A \# D\|_{L^{2}} \quad 2768$

Appendix A. Which Density Operator?

A.2. Time-Dependent Density Operators

\section{Introduction}

\subsection{Background}

Given a quantum system defined by the Hamiltonian $\hat{H}(x,-i \hbar \nabla)$ acting on $L^{2}\left(\mathbb{R}^{N}\right)$, the quantum canonical ensemble at the inverse temperature $\beta=1 /\left(k_{\mathrm{B}} T\right)$ is described by the density operator $\hat{\rho}=e^{-\beta \hat{H}}$. A quantum observable is defined by a Hermitian, densely defined operator $\hat{A}$ on $L^{2}\left(\mathbb{R}^{N}\right)$, and the quantum canonical ensemble average is obtained from the normalized trace of the product as $\frac{\operatorname{Tr}(\hat{\rho} \hat{A})}{\operatorname{Tr} \hat{\rho}}$. The Weyl quantization establishes a connection between the operator Hamiltonian $\hat{H}(x,-i \hbar \nabla)$ and its real-valued symbol function $H(x, p)$ defined on the classical phase space $\mathbb{R}^{N} \times \mathbb{R}^{N}$. The semiclassical analysis for $\hbar \rightarrow 0$ shows that the quantum observables can be approximated by the classical Gibbs ensemble average 


$$
\frac{\int_{\mathbb{R}^{N}} \int_{\mathbb{R}^{N}} A(x, p) e^{-\beta H(x, p)} \mathrm{d} x \mathrm{~d} p}{\int_{\mathbb{R}^{N}} \int_{\mathbb{R}^{N}} e^{-\beta H(x, p)} \mathrm{d} x \mathrm{~d} p},
$$

where the function $A(\cdot, \cdot)$ is the symbol of the Weyl- quantized operator $\hat{A}$.

The first mathematical result of such a semiclassical limit was obtained by Wigner [17]. Wigner introduced the "Wigner" function, based on solutions to the Schrödinger equation with scalar potentials, and made an expansion in the Planck constant $\hbar$ to relate the canonical quantum observable to the classical Gibbs phase-space average.

We study molecular dynamics approximations of canonical ensemble averages for quantum observables of the nuclei-electrons system. The role of the semiclassical parameter is played by the mass ratio $m_{\mathrm{e}} / m_{\mathrm{n}}$ between electrons mass $m_{\mathrm{e}}$ (the light particles) and the nuclei mass $m_{\mathrm{n}}$ (the heavy particles). We assume the atomic units (a.u.) in which $\hbar=1$ and the mass of electrons $m_{\mathrm{e}}=1$; thus, our small parameter is $1 / M \ll 1$ where $M$ is the mass of nuclei in atomic units. Note that in atomic units the proton mass is $m_{\mathrm{p}} \approx 1836$. The Boltzmann constant is $k_{\mathrm{B}}=1$ in atomic units hence $\beta=1 / T$.

The Hamiltonian of this system consists of the kinetic energy of nuclei and the electronic kinetic energy operator together with the operator describing interaction between electrons and nuclei

$$
\hat{H}=-\frac{1}{2 M} \Delta_{x}-\frac{1}{2} \Delta_{x_{e}}+\hat{V}_{e}\left(x, x_{e}\right) .
$$

In this work, we treat the electronic kinetic energy operator and the interaction operator, $\hat{H}_{e}=-\frac{1}{2} \Delta_{x_{e}}+\hat{V}_{e}\left(x, x_{e}\right)$ as a matrix-valued potential $V: \mathbb{R}^{N} \rightarrow \mathbb{C}^{d}$ defining the new Hamiltonian

$$
\hat{H}=-\frac{1}{2 M} \mathrm{I} \otimes \Delta+\hat{V}(x),
$$

where $\mathrm{I}$ is the $d \times d$ identity matrix. The matrix-valued potential $V$ is then obtained by approximating $\hat{H}_{e}$ on a finite-dimensional subspace of electronic states. We assume that this approximation results in the Hermitian matrixvalued confining potential $V$ with non-degenerate eigenvalues. By including the electron part as a matrix-valued operator, one can derive the limit as the electron-nuclei mass ratio $1 / M$ tends to zero, see [15] which, in Section 6 , includes an overview of previous results. This limit can then be approximated by ab initio molecular dynamics simulations for nuclei, with the potential generated by the electron eigenvalue problem, see $[10,12]$, based on the nuclei and electron scale separation using the Born-Oppenheimer approximation $[13,14]$. Such molecular dynamics simulations have the benefit to require less computational effort than to solve the Schrödinger equation with time-dependent electrons.

If the temperature is small in comparison with the minimal difference of the second and first eigenvalue of the electron potential $\hat{V}$, the probability for the quantum system to be in excited electron states is negligible and the canonical ab initio molecular dynamics based on the electron ground state yields accurate approximations. When the temperature is not small compared 
to this electron eigenvalue gap, the probability to be in excited states is substantial and the molecular dynamics associated with the electronic groundstate energy will not yield accurate approximation of quantum observables.

\subsection{Overview of Results}

We address an important question which seems mathematically open: How to modify the canonical ab initio molecular dynamics in order to accurately approximate quantum observables based on matrix- valued potentials and all temperatures?

We derive molecular dynamics methods that accurately, in mathematical sense, approximate a quantum observable also in the case where the temperature can be large compared to the first spectral gap. The approximation consists of a weighted sum of molecular dynamics observables for the scalar Hamiltonians which are the eigenvalues of the original matrix-valued Hamiltonian symbol. Furthermore, the weights, which are the probabilities to be in the corresponding electron states, are determined precisely as molecular dynamics observables. For instance, molecular systems with light atoms and applications with laser heating have been simulated more accurately by taking several electron states into account, see, e.g., [1,8] and Section 5.3.4 in [12].

Section 2 presents analysis with quantum observables not depending on time. In Sect. 3, we study observables that are correlations in time or depending on time correlations. The main result is that the weighted sums of molecular dynamics observables approximate canonical quantum observables, based on Schrödinger Hamiltonians with matrix-valued potentials for any positive temperature. The approximation error is bounded by the square root of electronnuclei mass ratio, $M^{-1 / 2}$, times a constant, provided the observable symbols are in the diagonal form with respect to the electron eigenstates and have up to three derivatives bounded in $L^{2}\left(\mathbb{R}^{N} \times \mathbb{R}^{N}\right)$, the Hamiltonian symbol has five derivatives and the electron eigenvalues are distinct for any nuclei position. An improved approximation error $\mathcal{O}\left(M^{-1}\right)$ holds with additional assumptions. The main mathematical tool is the semiclassical Weyl law, as formulated, for example, in $[15,18]$.

The semiclassical Weyl law has been used before, see [15], to approximate canonical quantum observables, based on general matrix-valued Hamiltonians, including infinite dimension $d=\infty$, by phase-space averages. For general Hamiltonian operators, the classical approximation with $\mathcal{O}\left(M^{-1}\right)$ accuracy includes $\mathcal{O}\left(M^{-1 / 2}\right)$ perturbations of the leading order Hamiltonian dynamics, as described in [15] by insightful analysis of the Hamiltonian flow based on perturbed Hamiltonian functions and symplectic forms.

With the aim to obtain sharper error estimates, we focus on a simpler case from the analysis perspective, when the nuclei kinetic energy contribution to the Hamiltonian operator is defined by the Laplacian, as in (1.2), and $d$ is finite. We exploit this simplified structure and the assumption of non-crossing electron eigenvalues, to construct global projections to the electron states related to the adiabatic approximation. We show how these projections, in the special case of the Hamiltonian (1.2), can be determined by a nonlinear eigenvalue 
problem solved by a power expansion provided by the Cauchy-Kovalevskaya theorem. This new approach with a nonlinear eigenvalue problem has an advantage in providing more precise error estimates. The construction is based on a convergent power series and not only on a semiclassical expansion which has been used to derive semiclassical limits elsewhere.

Another novelty of our approach is that our error estimates are not using the Calderon-Vaillancourt theorem that bounds the $L^{2}$ operator norm of a symbol by $L^{\infty}$ estimates of derivatives of order $N$ of the symbol. Instead, we take advantage of the Hamiltonian form (1.2) to derive error estimates that combine the Hilbert-Schmidt norm of quantum observables and Weyl's law to obtain new bounds in terms of the $L^{2}\left(\mathbb{R}^{N} \times \mathbb{R}^{N}\right)$ norm of remainder symbols based on three and five derivatives of the observables and the Hamiltonian, respectively. The new bounds of the remainder symbols given in Lemmas 3.11 and 3.12 are based on Hermitian properties of the Moyal composition. The constant in our $\mathcal{O}\left(M^{-1}\right)$ approximation result in Theorem 3.2 depends, for example, on the $L^{2}\left(\mathbb{R}^{N} \times \mathbb{R}^{N}\right)$ norm of three derivatives of the observable, while estimates based on the Calderon-Vaillancourt theorem require similar bounds on derivatives of order $N \gg 1$, which in practice can be very large so that $\mathcal{O}\left(M^{-1}\right)$ would not lead to useful error estimates for computational approximations with realistic values of $M$.

In conclusion, we make a step in the direction of precisely estimating constants for semiclassical approximations. There are two main new mathematical ideas that take advantage of the particular form of Hamiltonian (1.2): a nonlinear eigenvalue problem that avoids the traditional asymptotic expansion and an error estimate of remainder terms that use up to fourth-order derivatives only. We think these two ideas can be useful to further trace constants in situations with, for example, avoided crossings, degenerate and crossing electron eigenvalues, vanishing temperature and more general Hamiltonians.

At the core of the canonical ensemble is the Gibbs distribution, which has the important property that it is the marginal distribution for a subsystem weakly coupled to a heat bath, where the composite system is assumed to have the microcanonical distribution, see, e.g., [4]. In "Appendix A," we present a variant of this property, assuming instead that the marginal distribution of the subsystem is determined by the subsystem Hamiltonian. Another basic property is that the Gibbs density is a time-independent solution of the Liouville-von Neumann equation. "Appendix A" also includes a comparison of the classical and quantum Gibbs densities with respect to the classical and quantum Liouville equations. In Sect. 2.3 and "Appendix B," we present numerical results of simple model problems where the quantum and molecular dynamics observables are compared.

\section{The Schrödinger Equation and Gibbs Ensembles}

\subsection{Problem Formulation and Weyl Quantization}

We consider the matrix-valued Schrödinger operator 


$$
\hat{H}=-\frac{1}{2 M} \mathrm{I} \otimes \Delta+V(x)
$$

where $V: \mathbb{R}^{N} \rightarrow \mathbb{C}^{d \times d}$ is a Hermitian matrix-valued confining potential and $\mathrm{I} \otimes \Delta$ is the $d \times d$ identity matrix times the Laplacian on $\mathbb{R}^{N}$, modeling the nuclei kinetic energy.

Hence, the quantum model consists of $N / 3$ nuclei whose coordinates are in $\mathbb{R}^{3}$ and the wave functions $\Phi_{n} \in L^{2}\left(\mathbb{R}^{N}, \mathbb{C}^{d}\right)$ are vector-valued with $d$ components. We use the notation $\mathcal{H}:=L^{2}\left(\mathbb{R}^{N}, \mathbb{C}^{d}\right) \equiv\left[L^{2}\left(\mathbb{R}^{N}\right)\right]^{d}$. Approximation of the electronic part of the Hamiltonian using the electron eigenvalue problem gives the matrix potential (operator) $V(x)$ defined for each nuclei configuration $x \in \mathbb{R}^{N}$. Here $M \gg 1$ is the nuclei-electron mass ratio, assumed to be much larger than one. The setting with individual nuclei masses and a diagonal mass matrix $M$ can be transformed to form (2.1) by introducing the new coordinates $M_{1}^{1 / 2} \bar{x}=M^{1 / 2} x$, which transforms the Hamiltonian into

$$
-\frac{1}{2 M_{1}} \mathrm{I} \otimes \Delta_{\bar{x}}+V\left(M_{1}^{1 / 2} M^{-1 / 2} \bar{x}\right)
$$

To handle the spectrum of $\hat{H}$, we assume that the smallest eigenvalue $\lambda_{1}(x)$ of $V(x)$ tends to infinity as $|x| \rightarrow \infty$. This assumption implies that the spectrum of $\hat{H}$ is discrete, see [3]. More precisely, let $\Phi_{n}: \mathbb{R}^{N} \rightarrow \mathbb{C}^{d}$ and $E_{n} \in \mathbb{R}$ for $n=1,2,3, \ldots$ be solutions of the eigenvalue problem

$$
\hat{H} \Phi_{n}=E_{n} \Phi_{n}
$$

then the set of eigenfunctions $\left\{\Phi_{n}\right\}_{n=1}^{\infty}$ forms a complete basis of the Hilbert space $\mathcal{H}$.

To have a complete set of eigenfunctions in $\mathcal{H}$ is used for the analysis of the canonical quantum ensemble average in this work, although it is not crucial. The approach we present is based on the concept of the trace of quantum operators introduced by von Neumann [16].

Notation and Weyl Calculus Since our analysis provides error estimates for approximating quantum observables with classical ones, it is natural to use tools of Weyl calculus that defines correspondence between operators on $\mathcal{H}$ and their symbols in a suitable functional space. Here we introduce the notation used throughout this paper.

For functions $u, v \in \mathcal{H}$ we denote the scalar product

$$
\langle v, w\rangle=\int_{\mathbb{R}^{N}} v^{*}(x) \cdot w(x) \mathrm{d} x, \quad \text { where } v^{*}(x) \cdot w(x):=\sum_{j=1}^{d} v_{j}^{*}(x) w_{j}(x),
$$

and the corresponding norm $\|u\|_{\mathcal{H}}^{2}=\int_{\mathbb{R}^{N}}|u(x)|^{2} \mathrm{~d} x$. The space of smooth rapidly decaying matrix-valued functions, i.e., Schwartz space, is denoted $\mathcal{S}\left(\mathbb{R}^{N} \times \mathbb{R}^{N}, \mathbb{C}^{d \times d}\right)$ and it is abbreviated as $\mathcal{S}$. We define the Fourier transform $\mathcal{F}: A(x, p) \mapsto \mathcal{F}[A]\left(\xi_{x}, \xi_{p}\right)$ 


$$
\mathcal{F}[A]\left(\xi_{x}, \xi_{p}\right)=\int_{\mathbb{R}^{N}} \int_{\mathbb{R}^{N}} A\left(x^{\prime}, p^{\prime}\right) e^{-\mathrm{i}\left(x^{\prime} \cdot \xi_{x}+p^{\prime} \cdot \xi_{p}\right)} \mathrm{d} x^{\prime} \mathrm{d} p^{\prime} .
$$

We emphasize that the Fourier transform of a symbol $A$ is denoted by $\mathcal{F}[A]$ while $\hat{A}$ denotes the Weyl quantization of the symbol $A$. We also use the notation $(A)$ instead of the simple $\hat{A}$, in particular in long expressions such as $\left(A e^{-\beta H}\right)^{2}$. We define the Weyl quantization operator of the matrix-valued symbol $A \in \mathcal{S}$ as the mapping $A \mapsto \hat{A}$ that assigns to the symbol $A$ the linear operator $\hat{A}: \mathcal{H} \rightarrow \mathcal{H}$ defined for all $\Phi \in \mathcal{S}\left(\mathbb{R}^{N}, \mathbb{C}^{d}\right)$ by

$$
\hat{A} \Phi(x)=\left(\frac{\sqrt{M}}{2 \pi}\right)^{N} \int_{\mathbb{R}^{N}} \int_{\mathbb{R}^{N}} e^{\mathrm{i} M^{1 / 2}(x-y) \cdot p} A\left(\frac{1}{2}(x+y), p\right) \Phi(y) \mathrm{d} p \mathrm{~d} y .
$$

For example, the symbol $H(x, p):=\frac{1}{2}|p|^{2} \mathrm{I}+V(x)$ yields $\hat{H}=-(2 M)^{-1} \mathrm{I} \otimes \Delta+$ $V(x)$. Definition (2.3) implies that any quantization $\hat{A}$ is an integral operator

$$
\hat{A} \Phi(x)=\int_{\mathbb{R}^{N}} K_{A}(x, y) \Phi(y) \mathrm{d} y,
$$

with a matrix-valued, distributional kernel $K_{A}(x, y)$

$$
K_{A}(x, y)=\left(\frac{\sqrt{M}}{2 \pi}\right)^{N} \int_{\mathbb{R}^{N}} e^{\mathrm{i} M^{1 / 2}(x-y) \cdot p} A\left(\frac{1}{2}(x+y), p\right) \mathrm{d} p .
$$

The expression above shows that $K_{A}$ is the Fourier transform in the second argument of the symbol $A(x, p)$, and consequently, the Weyl quantization is well defined for symbols in $\mathcal{S}^{\prime}$, the space of tempered distributions.

An important property of the Weyl quantization is given by the Moyal product $A \# B$ of two symbols $A, B$

$$
[A \# B](x, p)=\left.e^{\frac{\mathrm{i}}{2 \sqrt{M}}\left(\nabla_{x^{\prime}} \cdot \nabla_{p}-\nabla_{x} \cdot \nabla_{p^{\prime}}\right)} A(x, p) B\left(x^{\prime}, p^{\prime}\right)\right|_{(x, p)=\left(x^{\prime}, p^{\prime}\right)} .
$$

The Moyal product provides correspondence between the algebra of operators and the algebra of their symbols by identifying composition of two operators with the Weyl quantization of the Moyal product of their symbols, more precisely

$$
(A \# B) \tilde{r}=(A) \tilde{(B)} .
$$

Further properties and background on the Weyl calculus are presented, for example, in $[6,18]$.

The principal idea in this work is to study the trace of operators on $\mathcal{H}$ with kernels $K_{A}$ defined by (2.4). The trace is a composition of the trace in $L^{2}\left(\mathbb{R}^{N}\right)$ and the trace of $d \times d$ matrices. The two different traces are defined by

$$
\begin{aligned}
& \operatorname{Tr} \hat{B}:=\sum_{n=1}^{\infty}\left\langle\Phi_{n}, \hat{B} \Phi_{n}\right\rangle, \text { for an operator } \hat{B} \text { on } \mathcal{H}, \\
& \operatorname{Tr} B:=\sum_{j=1}^{d} B_{j j}, \text { for a } d \times d \text { matrix } B .
\end{aligned}
$$


The analysis here uses the fact that the $\mathcal{H}$-trace of a Weyl operator based on a $d \times d$ matrix-valued symbol is equal to the phase-space average of its symbol trace. Indeed, we have by (2.4) for $A \in \mathcal{S}$

$$
\operatorname{Tr} \hat{A}=\int_{\mathbb{R}^{N}} \operatorname{Tr} K_{A}(x, x) \mathrm{d} x=\left(\frac{\sqrt{M}}{2 \pi}\right)^{N} \int_{\mathbb{R}^{N}} \int_{\mathbb{R}^{N}} \operatorname{Tr} A(x, p) \mathrm{d} p \mathrm{~d} x .
$$

We introduce the coordinate $z=(x, p) \in \mathbb{R}^{2 N}$ in the phase space and its Lebesgue measure $\mathrm{d} z=\mathrm{d} x \mathrm{~d} p$. In fact, also the composition of two Weyl operators is determined by the phase-space average as follows.

Lemma 2.1. The composition of two Weyl operators $\hat{A}$ and $\hat{B}$, with $A \in \mathcal{S}$ and $B \in \mathcal{S}$, satisfies

$$
\operatorname{Tr}(\hat{A} \hat{B})=\left(\frac{\sqrt{M}}{2 \pi}\right)^{N} \int_{\mathbb{R}^{2 N}} \operatorname{Tr}(A(z) B(z)) \mathrm{d} z,
$$

where $A(z) B(z)$ is the matrix product of the two $d \times d$ matrices $A(z)$ and $B(z)$.

Although this result is not new, see [15], we include a proof since it is important for the work here.

Proof. The kernel of the composition is

$$
\begin{aligned}
K_{A B}(x, y)= & \left(\frac{\sqrt{M}}{2 \pi}\right)^{2 N} \int_{\mathbb{R}^{3 N}} A\left(\frac{1}{2}\left(x+x^{\prime}\right), p\right) B\left(\frac{1}{2}\left(x^{\prime}+y\right), p^{\prime}\right) \\
& \times e^{i M^{1 / 2}\left(\left(x-x^{\prime}\right) \cdot p+\left(x^{\prime}-y\right) \cdot p^{\prime}\right)} \mathrm{d} p^{\prime} \mathrm{d} p \mathrm{~d} x^{\prime}
\end{aligned}
$$

so that the trace of the composition becomes

$$
\begin{aligned}
\operatorname{Tr}(\hat{A} \hat{B})=\int_{\mathbb{R}^{N}} \operatorname{Tr} K_{A B}(x, x) \mathrm{d} x \\
=\left(\frac{\sqrt{M}}{2 \pi}\right)^{2 N} \int_{\mathbb{R}^{4 N}} \operatorname{Tr}\left(A\left(\frac{1}{2}\left(x+x^{\prime}\right), p\right) B\left(\frac{1}{2}\left(x^{\prime}+x\right), p^{\prime}\right)\right) \\
\\
\times e^{i M^{1 / 2}\left(\left(x-x^{\prime}\right) \cdot p+\left(x^{\prime}-x\right) \cdot p^{\prime}\right)} \mathrm{d} p^{\prime} \mathrm{d} p \mathrm{~d} x^{\prime} \mathrm{d} x \\
=\left(\frac{\sqrt{M}}{2 \pi}\right)^{2 N} \int_{\mathbb{R}^{4 N}} \operatorname{Tr}\left(A(y, p) B\left(y, p^{\prime}\right)\right) e^{i M^{1 / 2} y^{\prime} \cdot\left(p-p^{\prime}\right)} \mathrm{d} p^{\prime} \mathrm{d} p \mathrm{~d} y^{\prime} \mathrm{d} y \\
=\left(\frac{\sqrt{M}}{2 \pi}\right)^{N} \int_{\mathbb{R}^{2 N}} \operatorname{Tr}(A(y, p) B(y, p)) \mathrm{d} p \mathrm{~d} y
\end{aligned}
$$

using the change of variables $\left(y, y^{\prime}\right)=\left(\left(x+x^{\prime}\right) / 2, x-x^{\prime}\right)$, which verifies the claim.

The isometry between Weyl operators with the Hilbert-Schmidt inner product, $\operatorname{Tr}\left(\hat{A}^{*} \hat{B}\right)$, and the corresponding $L^{2}\left(\mathbb{R}^{N} \times \mathbb{R}^{N}, \mathbb{C}^{d \times d}\right)$ symbols obtained by Lemma 2.1 shows how to extend from symbols in $\mathcal{S}$ to $L^{2}\left(\mathbb{R}^{N} \times\right.$ 
$\left.\mathbb{R}^{N}, \mathbb{C}^{d \times d}\right)$ by density of $\mathcal{S}$ in $L^{2}\left(\mathbb{R}^{N} \times \mathbb{R}^{N}, \mathbb{C}^{d \times d}\right)$, see [15]. We will use the Hilbert-Schmidt norm $\|\hat{A}\|_{\mathcal{H} \mathcal{S}}^{2}=\operatorname{Tr}\left(\hat{A}^{*} \hat{A}\right)=\operatorname{Tr}\left(\hat{A}^{2}\right)$, to estimate Weyl operators.

\subsection{Gibbs Density Operator and Its Approximation}

The quantum statistical average of the (time-independent) observable $\hat{A}$ in the canonical ensemble at the inverse temperature $\beta$ is given by

$$
\frac{\operatorname{Tr}(\hat{\rho} \hat{A})}{\operatorname{Tr}(\hat{\rho})} \text {, with (non-normalized) density operator } \hat{\rho}=e^{-\beta \hat{H}} \text {. }
$$

Similarly, the time-dependent or time-correlation observables become

$$
\frac{\operatorname{Tr}\left(\hat{A}_{\tau} \hat{B}_{0} \hat{\rho}\right)}{\operatorname{Tr}(\hat{\rho})}, \text { and related } \frac{\operatorname{Tr}\left(\hat{A}_{\tau}\left(\hat{B}_{0} \hat{\rho}+\hat{\rho} \hat{B}_{0}\right)\right)}{\operatorname{Tr}(\hat{\rho})},
$$

where $\hat{C}_{\tau}:=e^{\mathrm{i} \tau M^{1 / 2} \hat{H}} \hat{C} e^{-\mathrm{i} \tau M^{1 / 2} \hat{H}}$ is the quantum observable at time $\tau$ with $\hat{C}_{0}=\hat{C}$, as presented more precisely in Sect. 3 .

The Weyl quantization provides a correspondence between quantum operators $\hat{A}$ and their classical symbols $A$. The quantum canonical ensemble is described by the density matrix operator $\hat{\rho}=e^{-\beta \hat{H}}$, while the classical canonical ensemble is defined by the Gibbs distribution $\mu(\mathrm{d} z) \sim e^{-\beta H} \mathrm{~d} z$ on the phase space $z=(x, p)$.

Lemma 2.1 suggests that the correspondence between the quantum and classical Gibbs observables can be achieved if we use as the density matrix operator the Weyl quantization $\widehat{e^{-\beta H}}$. In Sect. 3, we derive error estimates that show that quantum observables such as those in (2.9) can be approximated by a classical Gibbs observables in (1.1), derived from the Hamiltonian symbol $H$, and thus linked to the classical molecular dynamics. Theorems 3.2, 3.6 and 3.7 prove error estimates both if the density operator used in $(2.9)$ is $\hat{\rho}=e^{-\beta \hat{H}}$ and if it is replaced with Weyl quantization $\widehat{e^{-\beta H}}$.

Replacing the standard density operator $e^{-\beta \hat{H}}$ with the operator $\widehat{e^{-\beta H}}$ raises a question which one should be taken as a reference for error analysis. We show in the proof of Theorem 3.6 that the observables based on these two operators differ by a quantity of order $\mathcal{O}\left(M^{-1}\right)$ when the number of particles, $N$, is small compared to $M$. Thus, in this case one can use either of them. However, the standard density operator $e^{-\beta \hat{H}}$ is the stationary solution of the (quantum) Liouville-von Neumann equation, while the corresponding symbol is not the time-independent solution of the classical Liouville equation. On the other hand, starting with the classical Gibbs density $e^{-\beta H}$ the corresponding Weyl quantization gives the proposed density operator $\widehat{e^{-\beta H}}$ which is not a stationary solution of the Liouville--von Neumann equation. We discuss this issue in "Appendix A."

The trace property of Lemma 2.1 shows that an approximation of the Gibbs observable, where the order of the operations of exponentiation and quantization has been reversed, satisfies 


$$
\begin{aligned}
\operatorname{Tr}\left(\widehat{e^{-\beta H}} \hat{A}\right) & =\sum_{n=1}^{\infty}\left\langle\Phi_{n}, \widehat{e^{-\beta H}} \hat{A} \Phi_{n}\right\rangle \\
& =\left(\frac{\sqrt{M}}{2 \pi}\right)^{N} \int_{\mathbb{R}^{N}} \operatorname{Tr}\left(A(z) e^{-\beta H(z)}\right) \mathrm{d} z
\end{aligned}
$$

and in the normalized form

$$
G:=\frac{\operatorname{Tr}\left(\widehat{e^{-\beta H}} \hat{A}\right)}{\operatorname{Tr}\left(\widehat{e^{-\beta H}}\right)}=\frac{\int_{\mathbb{R}^{2 N}} \operatorname{Tr}\left(A(z) e^{-\beta H(z)}\right) \mathrm{d} z}{\int_{\mathbb{R}^{2 N}} \operatorname{Tr}\left(e^{-\beta H(z)}\right) \mathrm{d} z} .
$$

We will use the diagonalized form of the Hamiltonian symbol and similarly of transformed observables. More precisely, let $\tilde{A}: \mathbb{R}^{2 N} \rightarrow \mathbb{C}^{d \times d}$ be a symbol in the Schwartz class and consider symbols in the matrix product form $A(z)=$ $\tilde{\Psi}(x) \tilde{A}(z) \tilde{\Psi}^{*}(x)$, where $\tilde{\Psi}(x)$ is the $d \times d$ matrix composed of the eigenvectors to $V(x)$ as columns, i.e.,

$$
\sum_{j=1}^{d} V_{i j}(x) \tilde{\Psi}_{j k}(x)=\tilde{\lambda}_{k}(x) \tilde{\Psi}_{i k}(x),
$$

and $\tilde{\lambda}_{k}(x), k=1, \ldots, d$, denote the eigenvalues of $V(x)$ in the increasing order. Here $\tilde{\Psi}^{*}(x)$ is the Hermitian adjoint of $\tilde{\Psi}(x)$ in $\mathbb{C}^{d}$ and $\delta_{j k}$ is the Kronecker delta. Then $\tilde{\Psi}(x)$ diagonalizes $H(z)$ and it and $A(z)$ satisfy:

$$
\begin{array}{ll}
H(z)=\tilde{\Psi}(x) \tilde{H}(z) \tilde{\Psi}^{*}(x), & \text { where } \tilde{H}_{j k}(z)=\delta_{j k}\left(\frac{|p|^{2}}{2}+\tilde{\lambda}_{j}(x)\right), \\
A(z)=\tilde{\Psi}(x) \tilde{A}(z) \tilde{\Psi}^{*}(x), \quad \text { where } \tilde{A}(z)=\tilde{\Psi}^{*}(x) A(z) \tilde{\Psi}(x) .
\end{array}
$$

The diagonal property of $e^{-\beta \tilde{H}}$ shows that the trace satisfies

$$
\operatorname{Tr}\left(A(z) e^{-\beta H(z)}\right)=\operatorname{Tr}\left(\tilde{A}(z) e^{-\beta \tilde{H}(z)}\right)=\sum_{j=1}^{d} \tilde{A}_{j j}(z) e^{-\beta \tilde{H}_{j j}(z)},
$$

which only requires the diagonal part of $\tilde{A}$. We obtain by $(2.10)$ the approximate Gibbs quantum observable as a sum

$$
G=\frac{\sum_{j=1}^{d} \int_{\mathbb{R}^{2 N}} \tilde{A}_{j j}(z) e^{-\beta \tilde{H}_{j j}(z)} \mathrm{d} z}{\sum_{j=1}^{d} \int_{\mathbb{R}^{2 N}} e^{-\beta \tilde{H}_{j j}(z)} \mathrm{d} z}
$$

and each term can be written in canonical ensemble form:

Lemma 2.2. The approximate canonical ensemble average satisfies, for $\beta>0$,

$$
\frac{\operatorname{Tr}\left(\widehat{e^{-\beta H}} \hat{A}\right)}{\operatorname{Tr}\left(\widehat{e^{-\beta H}}\right)}=\sum_{j=1}^{d} q_{j} \int_{\mathbb{R}^{2 N}} \tilde{A}_{j j}(z) \frac{e^{-\beta \tilde{H}_{j j}(z)} \mathrm{d} z}{\int_{\mathbb{R}^{2 N}} e^{-\beta \tilde{H}_{j j}\left(z^{\prime}\right)} \mathrm{d} z^{\prime}}
$$

with the weights given by respective probability to be in the state $j$

$$
q_{j}=q_{j}(\tilde{H}):=\frac{\int_{\mathbb{R}^{2 N}} e^{-\beta \tilde{H}_{j j}(z)} \mathrm{d} z}{\sum_{k=1}^{d} \int_{\mathbb{R}^{2 N}} e^{-\beta \tilde{H}_{k k}\left(z^{\prime}\right)} \mathrm{d} z^{\prime}}, \quad j=1, \ldots, d .
$$


In Sect. 3, we analyze the trace based on the time-independent Gibbs density operator $e^{-\beta \hat{H}}$ and on $\widehat{e^{-\beta H}}$, using instead the transformation $\hat{A}=$ $\hat{\Psi} \hat{\bar{A}} \hat{\Psi}^{*}$ for a diagonal symbol $\bar{A}:[0, \infty) \times \mathbb{R}^{2 N} \rightarrow \mathbb{C}^{d \times d}$ with an orthogonal matrix $\Psi: \mathbb{R}^{N} \rightarrow \mathbb{C}^{d \times d}$ diagonalizing a nonlinear perturbation of the eigenvalue problem (2.11).

Remark 2.3. Since $\Psi_{\cdot j}\left(\Psi_{\cdot j}\right)^{*}$ is the projection to the electron state $j$, a method to handle projections to electron states is to normalize with respect to that state and use

$$
\frac{\sum_{n=1}^{\infty}\left\langle\Phi_{n},\left(\Psi_{\cdot j} \tilde{A}_{j j}\left(\Psi_{\cdot j}\right)^{*} \hat{)}\left(e^{-\beta H} \hat{)} \Phi_{n}\right\rangle\right.\right.}{\sum_{n=1}^{\infty}\left\langle\Phi_{n},\left(\Psi \cdot j(\Psi \cdot j)^{*}\right) \hat{\left.\left(e^{-\beta H}\right) \hat{\Phi_{n}}\right\rangle}\right.}=\int_{\mathbb{R}^{2 N}} \tilde{A}_{j j}(z) \frac{e^{-\beta \tilde{H}_{j j}(z)}}{\int_{\mathbb{R}^{2 N}} e^{-\beta \tilde{H}_{j j}(\bar{z})} d \bar{z}} \mathrm{~d} z .
$$

\subsection{Computational Demonstration}

In order to demonstrate computational consequences of the presented analysis, we formulate a simple model problem for comparing quantum observables with observables obtained from molecular dynamics. We consider a model Hamiltonian $\hat{H}=-M^{-1} \mathrm{I} \otimes \Delta+V(x)$, where $V: \mathbb{R} \rightarrow \mathbb{R}^{2 \times 2}$ and $\mathrm{I}$ is the $2 \times 2$ identity matrix. The corresponding Schrödinger eigenvalue equation $\hat{H} \Phi_{n}=$ $E_{n} \Phi_{n}$, where $E_{n} \in \mathbb{R}$ and $\Phi_{n}: \mathbb{R} \rightarrow \mathbb{R}^{2}$, represents a model with a heavy particle with coordinate $x \in \mathbb{R}$ and a two-state light electron particle. The details of the example, numerical implementation, and specific values of the parameters are described in "Appendix B."

2.3.1. Equilibrium Observables. First we focus on equilibrium position observables. We demonstrate numerically an estimate of the difference of the quantum and classical canonical observables, given by (2.8) and the righthand side in (2.13), respectively. We view the averaging for the quantum, $\int_{\mathbb{R}} A(x) \mu_{\mathrm{qc}}(x) \mathrm{d} x$, and classical, $\int_{\mathbb{R}} A(x) \mu_{\mathrm{cl}}(x) \mathrm{d} x$, position observables as averaging with respect to the measures with the densities

$$
\begin{aligned}
\mu_{\mathrm{qc}}(x) & =\frac{\sum_{n}\left|\Phi_{n}(x)\right|^{2} e^{-\beta E_{n}}}{\int_{\mathbb{R}} \sum_{n}\left|\Phi_{n}\left(x^{\prime}\right)\right|^{2} e^{-\beta E_{n}} \mathrm{~d} x^{\prime}}, \\
\mu_{\mathrm{cl}}(x) & =\sum_{k=1}^{2} q_{k} \frac{e^{-\beta \lambda_{k}(x)}}{\int_{\mathbb{R}} e^{-\beta \lambda_{k}\left(x^{\prime}\right)} \mathrm{d} x^{\prime}}, \quad q_{k}=\frac{\int_{\mathbb{R}} e^{-\beta \lambda_{k}(x)} \mathrm{d} x}{\sum_{j=1}^{2} \int_{\mathbb{R}} e^{-\beta \lambda_{j}(x)} \mathrm{d} x} .
\end{aligned}
$$

Here $\left(E_{n}, \Phi_{n}(x)\right)$ denotes an eigenpair of the operator $\hat{H}$ and $\lambda_{k}(x), k=1,2$ is the $k$ th eigenvalue of the matrix-valued function $V(x)$, such that $\lambda_{1}(x) \leq$ $\lambda_{2}(x)$.

Figure 1a shows the classical density $\mu_{\mathrm{cl}}$ from molecular dynamics on the electron ground state, corresponding to $q_{1}=1$ and $q_{2}=0$, and the density given by the values of $q_{k}$ in (2.16). For the choice of parameters, these probabilities are $q_{1}=0.8$ and $q_{2}=0.2$. Figure 2 a shows the Schrödinger and classical densities $\mu_{\mathrm{qc}}(x)$ and $\mu_{\mathrm{cl}}(x)$, computed using (2.15) and (2.16). We note that the classical density computed using (2.16) approximates the quantum density quite well, whereas Fig. 1a shows that the classical density computed using 

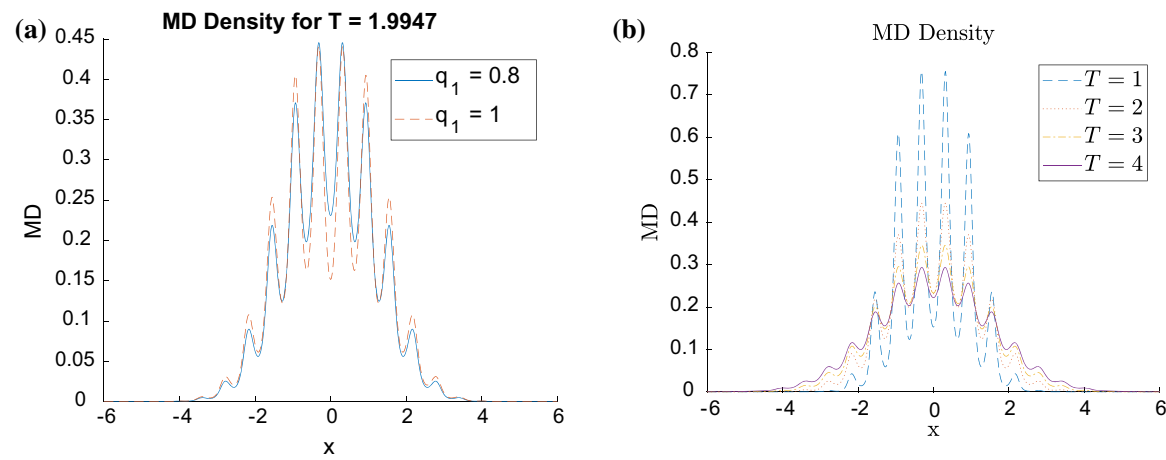

Figure 1. a The classical density $\mu_{\mathrm{cl}}$ using $q_{1}=1$, corresponding to molecular dynamics on the canonical ground state at $T=1.9947$ and $M=1000$ compared to the classical density with $q_{1}=0.8$, from (2.16), b molecular dynamics density (2.16) for different temperatures $T$ and $M=1000$.
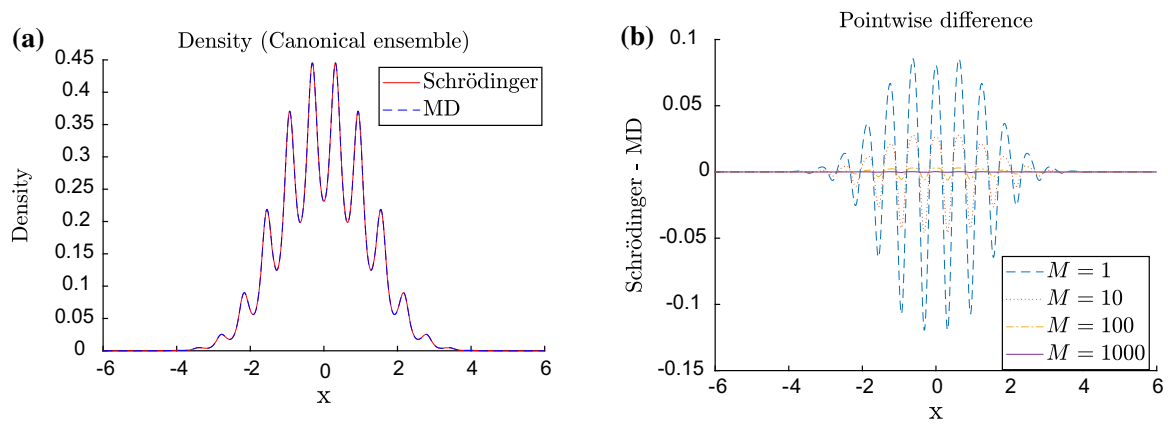

Figure 2. a The quantum and classical densities in (2.15) and (2.16) plotted for the same parameters $M=1000$ and $T=1.9947, \mathbf{b}$ difference of the classical and quantum pointwise densities, given in (2.16) and (2.15), for different mass ratio $M$ at $T=1.9947$ (right).

only the electron ground state is not close to the quantum density. The potential $V(x)$ that was used in these numerical tests is described in "Appendix B" and has eigenvalues $\lambda_{k}(x)$ depicted in Fig. 3.

Results from numerical simulations demonstrate the error analysis of Sect. 3, in particular that the $L^{1}$ as well as $L^{\infty}$ difference between $\mu_{\mathrm{qc}}$ and $\mu_{\mathrm{cl}}$ decreases as $\mathcal{O}\left(M^{-1}\right)$. We note the striking agreement between the Schrödinger and molecular dynamics equilibrium densities for $M=1000$ in Fig. 2a. The pointwise difference for $M=1,10,100,1000$ is given in Fig. 2b. Figure 1b shows the increasing variation of the molecular dynamics density as the temperature decreases. We conclude that although the densities vary substantially for different temperatures, the molecular dynamics density approximates the 


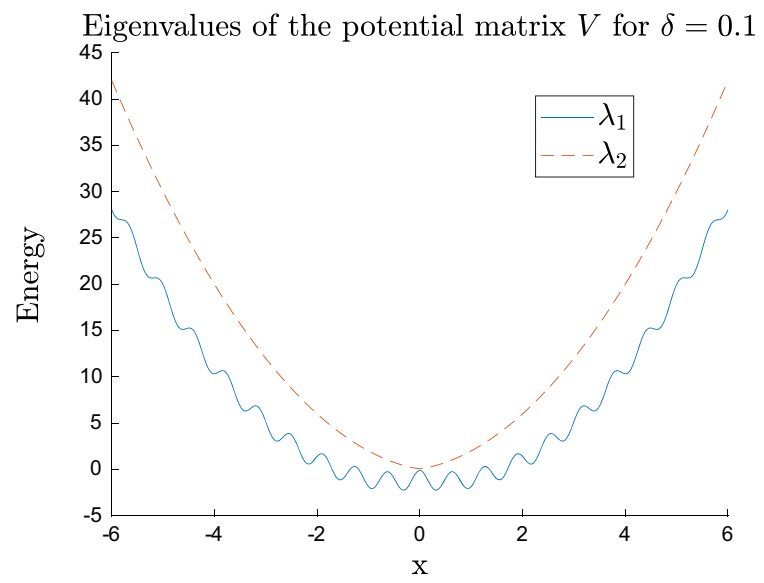

FigurE 3. The eigenvalue functions $\lambda_{1}(x)$ and $\lambda_{2}(x)$ of $V(x)$.

canonical Schrödinger density well, with the error $\mathcal{O}\left(M^{-1}\right)$ shown also in Fig. 4. Molecular dynamics approximation of quantum observables in the microcanonical ensemble typically has larger errors, see [2].

Figure 4 depicts the $\mathcal{O}\left(M^{-1}\right)$ decrease in the error for different values of the spectral gap between the ground state $\lambda_{1}(x)$ and the next state $\lambda_{2}(x)$ at a fixed temperature. Both errors are inverse proportional to the mass ratio $M$. The temperature $T$ and the spectral gap (controlled by the parameter $\delta$ ) are chosen so that the weight $q_{1}=0.8$; in other words, the excited state contributes non-trivially to the average. In neither of the norms, in Fig. 4, can we see a $\delta$ dependency in the error while in our derivation the constant in $\mathcal{O}\left(M^{-1}\right)$ of $(2.17)$ depends on the norm of the derivatives up to order five of the eigenvectors and eigenvalues of $V$. In this example, the eigenvector derivative of the order $n$ is $\mathcal{O}\left(\delta^{-n}\right)$.

2.3.2. Time-Correlated Observables. Next we demonstrate that the derived method and analysis are also applicable to observables depending on time correlations. In particular, we test the position observable $x_{0}$ at time $t=0$ with the position observable $x_{\tau}$ at time $t=\tau$. The time evolution of the position observable operator is given, in Heisenberg representation, as

$$
\hat{x}_{\tau}:=e^{\mathrm{i} \tau \sqrt{M} \hat{H}} \hat{x}_{0} e^{-\mathrm{i} \tau \sqrt{M} \hat{H}} .
$$

Applied to the special case in this example, with $\hat{H}$ as in Sect. 2.3.1, Theorems $3.2,3.6$ and 3.7 show that

$$
\begin{aligned}
& \frac{\frac{1}{2} \operatorname{Tr}\left(\hat{x}_{\tau}\left(\hat{x}_{0} e^{-\beta \hat{H}}+e^{-\beta \hat{H}} \hat{x}_{0}\right)\right)}{\operatorname{Tr}\left(e^{-\beta \hat{H}}\right)} \\
& \quad=\sum_{j=1}^{2} \int_{\mathbb{R}^{2}} q_{j} x_{\tau}^{j}\left(z_{0}\right) x_{0}^{j}\left(z_{0}\right) \frac{e^{-\beta\left(\frac{\left|p_{0}\right|^{2}}{2}+\lambda_{j}\left(x_{0}\right)\right)}}{\int_{\mathbb{R}^{2}} e^{-\beta\left(\frac{|p|^{2}}{2}+\lambda_{j}(x)\right)} \mathrm{d} z} \mathrm{~d} z_{0}+\mathcal{O}\left(M^{-1}\right),
\end{aligned}
$$



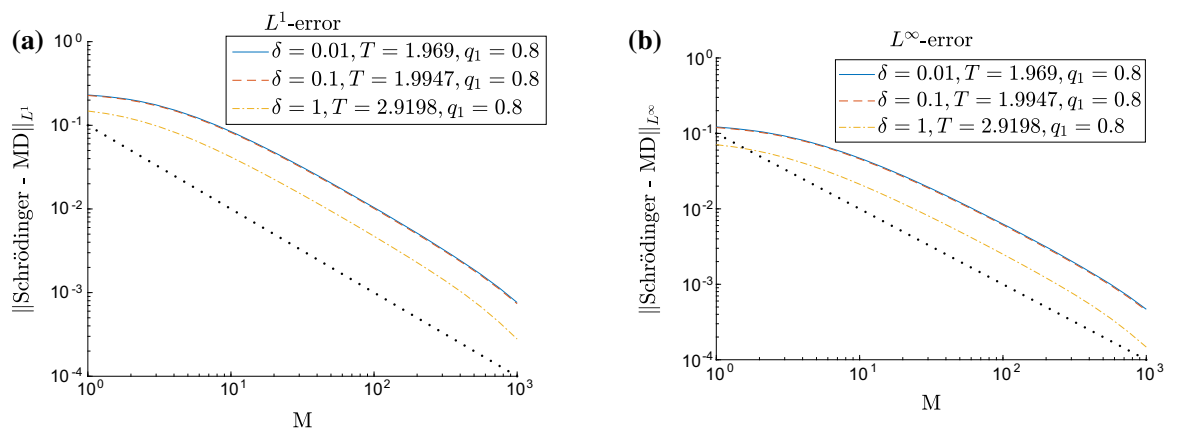

Figure 4. Dependence on the mass $M$ of the error between quantum and molecular dynamics densities $\mu_{\mathrm{qc}}$ and $\mu_{\mathrm{cl}}$, respectively, shown in $\log -\log$ scale. The dotted lines show the reference slope -1 . See "Appendix B" for details on the choice of the parameter $\delta$ and the temperature T. a $L^{1}$ error, b $L^{\infty}$ error
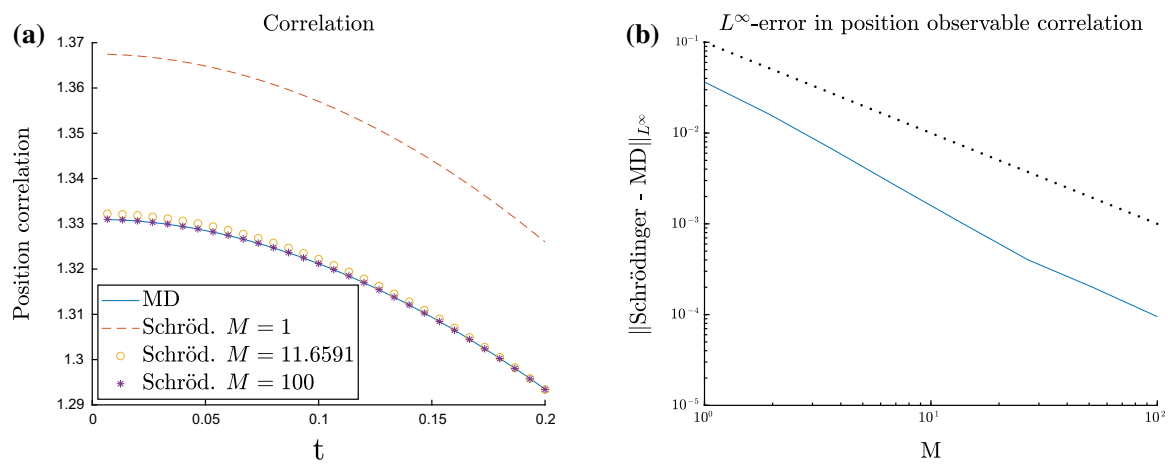

Figure 5. a Molecular dynamics position correlation observable shown together with its Schrödinger counterpart, for three values of the mass ratio $M, \mathbf{b}$ the $L^{\infty}$-error in the molecular dynamics position correlation observable approximation for $\tau=0.2$, shown in $\log -\log$ scale. The dotted line shows the slope -1 for reference

where $z_{\tau}^{j}=\left(x_{\tau}^{j}, p_{\tau}^{j}\right), j=1,2$ solve the Hamiltonian dynamics

$$
\left\{\begin{array}{l}
\dot{x}_{\tau}^{j}=p_{\tau}^{j} \\
\dot{p}_{\tau}^{j}=-\frac{\mathrm{d}}{\mathrm{d} x_{\tau}^{j}} \lambda_{j}\left(x_{\tau}^{j}\right), \quad \tau>0
\end{array}\right.
$$

with the initial condition $z_{0}^{j}=\left(x_{0}, p_{0}\right)=z_{0}$, and $\lambda_{j}$ as in Fig. 3, precisely defined in "Appendix B.1".

With the same values for $T$ and $\delta$ as in the case of equilibrium observables, we show the position correlation observable for different correlation times $\tau$ and three different mass ratios $M$, in Fig. 5a. 
Figure $5 \mathrm{~b}$ demonstrates that the $L^{\infty}$-error is inversely proportional to the mass ratio $M$ in agreement with the error analysis of Sect. 3 .

\section{Time-Correlated Observables}

In this section, we study canonical quantum observables for correlations in time, namely

$$
\operatorname{Tr}\left(\hat{A}_{\tau} \hat{B}_{0} e^{-\beta \hat{H}}\right)=\sum_{n=1}^{\infty}\left\langle\Phi_{n}, \hat{A}_{\tau} \hat{B}_{0} e^{-\beta \hat{H}} \Phi_{n}\right\rangle,
$$

and the related variant $\operatorname{Tr}\left(\hat{A}_{\tau}\left(\hat{B}_{0} e^{-\beta \hat{H}}+e^{-\beta \hat{H}} \hat{B}_{0}\right)\right)$, based on the timedependent operator $\hat{A}_{\tau}$, which for $\tau \in \mathbb{R}$ is defined by

$$
\hat{A}_{\tau}:=e^{\mathrm{i} \tau M^{1 / 2} \hat{H}} \hat{A}_{0} e^{-\mathrm{i} \tau M^{1 / 2} \hat{H}},
$$

with a matrix-valued symbol $A_{0}: \mathbb{R}^{2 N} \rightarrow \mathbb{C}^{d \times d}$ in the Schwartz class.

Example For instance, the observable for the diffusion constant

$$
\frac{1}{6 \tau} \frac{3}{N} \sum_{k=1}^{N / 3}\left|x_{k}(\tau)-x_{k}(0)\right|^{2}=\frac{1}{2 N \tau}\left(|x(\tau)|^{2}+|x(0)|^{2}-2 x(\tau) \cdot x(0)\right)
$$

uses the time correlation $\hat{x}(\tau) \cdot \hat{x}(0)$ where $\hat{A}_{\tau}=\hat{x}_{\tau} \mathrm{I}$ and $\hat{B}_{0}=\hat{x}_{0} \mathrm{I}$ and

$$
\hat{x}_{\tau} \cdot \hat{x}_{0}=\sum_{n=1}^{N / 3} \sum_{j=1}^{3} e^{\mathrm{i} \tau M^{1 / 2} \hat{H}} \hat{x}_{n_{j}} e^{-\mathrm{i} \tau M^{1 / 2}} \hat{H}_{n_{n_{j}}} .
$$

To analyze the time evolution of $\hat{A}_{\tau}$, we use transformed variables: assume that $\Psi: \mathbb{R}^{N} \rightarrow \mathbb{C}^{d \times d}$ and $\Psi(x)$ is an orthogonal matrix with the Hermitian transpose $\Psi^{*}(x)$ and define $\bar{A}:[0, \infty) \times \mathbb{R}^{2 N} \rightarrow \mathbb{C}^{d \times d}$ by

$$
\widehat{\bar{A}(\tau, z)}=\hat{\Psi}^{*}(x) \hat{A}_{\tau} \hat{\Psi}(x), \quad \tau \geq 0 .
$$

The matrix $\Psi(x)$ we will use is defined precisely below and it approximates the matrix $\tilde{\Psi}(x)$ that diagonalizes the potential matrix $V(x)$ in the sense that $\|\Psi(x)-\tilde{\Psi}(x)\|=\mathcal{O}\left(M^{-1}\right)$. We also assume that

$$
\hat{B_{0}}=\hat{\Psi} \widehat{\bar{B}_{0}} \hat{\Psi}^{*}
$$

and we restrict our study to the case where the $d \times d$ matrix symbols $\bar{A}(0, \cdot)=$ $\bar{A}_{0}$ and $\bar{B}_{0}$ are diagonal, as motivated in (3.7). Let $\alpha$ be any complex number and define for $t \in \mathbb{R}$ the exponential

$$
\widehat{\bar{y}_{t}}:=\hat{\Psi}^{*} e^{t \alpha \hat{H}} \hat{\Psi} .
$$

Differentiation shows that

$$
\partial_{t} \widehat{\bar{y}}_{t}=\alpha \hat{\Psi}^{*} \hat{H} \hat{\Psi} \hat{\Psi}^{*} e^{t \alpha \hat{H}} \hat{\Psi}=\alpha \hat{\Psi}^{*} \hat{H} \hat{\Psi} \widehat{\bar{y}}_{t}
$$

and we conclude that

$$
\widehat{\bar{y}}_{t}=e^{t \alpha \hat{\Psi}^{*} \hat{H}} \hat{\Psi} .
$$


The composition rule

$$
\begin{aligned}
A \# B(x, p) & :=\left.e^{\frac{\mathrm{i}}{2 M^{1 / 2}}\left(\nabla_{x^{\prime}} \cdot \nabla_{p}-\nabla_{x} \cdot \nabla_{p^{\prime}}\right)} A(x, p) B\left(x^{\prime}, p^{\prime}\right)\right|_{(x, p)=\left(x^{\prime}, p^{\prime}\right)}, \\
\hat{A} \hat{B} & =\widehat{A \# B}
\end{aligned}
$$

see Theorems 4.11 and 4.12 in [18], implies

$$
\hat{\Psi}^{*} \hat{H} \hat{\Psi}=\left(\Psi^{*} \# H \# \Psi \hat{)} .\right.
$$

A power expansion of the exponential in (3.5), see [18], yields the semiclassical expansion

$$
\left.\sum_{n=0}^{m} \frac{1}{n !}\left(\mathrm{i} \frac{\nabla_{x^{\prime}} \cdot \nabla_{p}-\nabla_{x} \cdot \nabla_{p^{\prime}}}{2 M^{1 / 2}}\right)^{n} A(x, p) B\left(x^{\prime}, p^{\prime}\right)\right|_{(x, p)=\left(x^{\prime}, p^{\prime}\right)}+\mathcal{O}\left(M^{-(m+1)}\right) .
$$

This expansion is defined for symbols in the Schwartz class, and Lemma 3.11 provides an extension to a larger set of symbols. Due to the special form of the Hamiltonian studied here, namely $H(x, p)=\frac{|p|^{2}}{2} \mathrm{I}+V(x)$, all terms of the semiclassical expansion for (3.6) with higher powers than $M^{-1}$ drop out, and we obtain a simple sum of only two terms:

Lemma 3.1. Any orthogonal two times differentiable matrix-valued mapping $\Psi: \mathbb{R}^{N} \rightarrow \mathbb{C}^{d \times d}$ satisfies

$$
\Psi^{*} \# H \# \Psi(x, p)=\Psi^{*}(x) H(x, p) \Psi(x)+\frac{1}{4 M} \nabla \Psi^{*}(x) \cdot \nabla \Psi(x) .
$$

Proof. The composition in (3.5) shows that

$$
\begin{aligned}
\Psi^{*} & \# \# \Psi(x, p) \\
= & \Psi^{*}(x) \#\left(H(x, p) \Psi(x)+\frac{\mathrm{i} M^{-1 / 2}}{2} p \cdot \nabla \Psi(x)-\frac{M^{-1}}{4} \Delta \Psi(x)\right) \\
= & \Psi^{*}(x) H(x, p) \Psi(x)+\frac{\mathrm{i} M^{-1 / 2}}{2} p \cdot \nabla \Psi^{*}(x) \Psi(x)-\frac{M^{-1}}{4} \Delta \Psi^{*}(x) \Psi(x) \\
& +\frac{\mathrm{i}}{2 M^{1 / 2}} \Psi^{*}(x) p \cdot \nabla \Psi(x)-\frac{1}{4 M} \nabla \Psi^{*}(x) \cdot \nabla \Psi(x)-\frac{1}{4 M} \Psi^{*}(x) \Delta \Psi(x)
\end{aligned}
$$

which by the property $\Psi^{*} \Psi=\mathrm{I}$ can be written

$$
\begin{aligned}
\Psi^{*} \# H \# \Psi(x, p)= & \Psi^{*}(x) H(x, p) \Psi(x)+\frac{\mathrm{i}}{2 M^{1 / 2}} p \cdot \nabla\left(\Psi^{*}(x) \Psi(x)\right) \\
& -\frac{1}{4 M}\left(\Delta\left(\Psi^{*}(x) \Psi(x)\right)-\nabla \Psi^{*}(x) \cdot \nabla \Psi(x)\right) \\
= & \Psi^{*}(x) H(x, p) \Psi(x)+\frac{1}{4 M} \nabla \Psi^{*}(x) \cdot \nabla \Psi(x) .
\end{aligned}
$$

Define

$$
\bar{H}(x, p):=\Psi^{*} \# H \# \Psi=\Psi^{*}(x) H(x, p) \Psi(x)+\frac{1}{4 M} \nabla \Psi^{*}(x) \cdot \nabla \Psi(x),
$$


which by (3.3) and (3.4) implies $\hat{\Psi}^{*} e^{t \alpha \hat{H}} \hat{\Psi}=e^{t \alpha \hat{\bar{H}}}$ and we obtain by (3.1) and (3.2) that

$$
\partial_{t} \hat{\bar{A}}_{t}=\mathrm{i} M^{1 / 2}\left[\hat{\bar{H}}, \hat{\bar{A}}_{t}\right]
$$

The next step is to determine $\Psi$ so that $\bar{H}=\Psi^{*} \# H \# \Psi$ is diagonal [or approximately diagonal in (3.18)], in order to make $\bar{H} \bar{A}_{t}-\bar{A}_{t} \bar{H}$ small since it appears in the expansion of the compositions in

$$
\partial_{t} \bar{A}_{t}=\mathrm{i} M^{1 / 2}\left(\bar{H} \# \bar{A}_{t}-\bar{A}_{t} \# \bar{H}\right) .
$$

To have $\bar{H} \bar{A}_{t}-\bar{A}_{t} \bar{H}$ small then also requires $\bar{A}_{t}$ to be diagonal (or almost diagonal). In the case when $\bar{H}$ is diagonal, the quantization $\hat{\bar{H}}$ is diagonal and then $\hat{\bar{A}}$ remains diagonal if it initially is diagonal, since then

$$
\frac{\mathrm{d}}{\mathrm{d} t} \hat{\bar{A}}_{j k}(t)=\mathrm{i} M^{1 / 2}\left(\bar{H}_{j j} \hat{\bar{A}}_{j k}(t)-\hat{\bar{A}}_{j k}(t) \bar{H}_{k k}\right)=0, \text { for } j \neq k \text {. }
$$

Consequently, we restrict our study to observables where $\bar{A}_{0}=\bar{A}(0)$ are diagonal.

We have

$$
\bar{H}(x, p)=\Psi^{*}(x)\left(\frac{|p|^{2}}{2} \mathrm{I}+V(x)+\frac{1}{4 M} \Psi(x) \nabla \Psi^{*}(x) \cdot \nabla \Psi(x) \Psi^{*}(x)\right) \Psi(x) .
$$

Therefore, the aim is to choose the orthogonal matrix $\Psi$ so that it is a solution or an approximate solution to the nonlinear eigenvalue problem

$$
\left(V+\frac{1}{4 M} \Psi \nabla \Psi^{*} \cdot \nabla \Psi \Psi^{*}\right) \Psi=\Psi \Lambda
$$

where $\Lambda$ is diagonal with $\Lambda_{j j}=: \lambda_{j}$. Such a transformation $\Psi$ is an $\mathcal{O}\left(M^{-1}\right)$ perturbation of the eigenvectors to $V(x)$, provided the eigenvalues $\tilde{\lambda}_{j}(x)$ of $V(x)$ do not cross and $M$ is sufficiently large. Section 3.1 shows that (3.8) has a unique solution, if the potential $V$ is real analytic, and that the solution can be approximated by an asymptotic expansion.

\subsection{Solution of the Nonlinear Eigenvalue Problem}

This section presents a version of the standard regular perturbation analysis of matrix eigenvalue problems, cf. [9], which shows that the nonlinear eigenvalue problem (3.8) can be written as a nonlinear system of first-order partial differential equations solved by a power expansion provided by the CauchyKovalevskaya theorem or by an asymptotic expansion.

We define for small $\epsilon \in \mathbb{R}$ the matrix

$$
\breve{V}(\epsilon):=\breve{V}+\epsilon \breve{B}
$$

where $\breve{V}$ and $\breve{B}$ are real symmetric $d \times d$ matrices, depending on a parameter $x \in \mathbb{R}^{N}$ and on another parameter $M \in \mathbb{R}$. The nonlinear eigenvalue problem (3.8) can then be written as

$$
\begin{aligned}
& \breve{V}\left(\frac{1}{4 M}\right) \Psi=\Psi \Lambda, \\
& \breve{B}(x):=\Psi(x) \nabla \Psi^{*}(x) \cdot \nabla \Psi(x) \Psi^{*}(x) .
\end{aligned}
$$


We assume that the matrices $\breve{V}$ and $\breve{B}$ are $m$ times continuously differentiable as a function of $x$. Differentiating with respect to $\epsilon$, the eigenvalue problem

$$
\breve{V}(\epsilon) \psi_{k}(\epsilon)=\lambda_{k}(\epsilon) \psi_{k}(\epsilon)
$$

with the eigenvalues $\lambda_{k}(\epsilon) \in \mathbb{R}$ and the corresponding normalized real-valued eigenvectors $\psi_{k}(\epsilon) \in \mathbb{R}^{d}, k=1, \ldots d$, we obtain

$$
\left(\breve{V}(\epsilon)-\lambda_{k}(\epsilon)\right) \psi_{k}^{\prime}(\epsilon)=-\left(\breve{V}^{\prime}(\epsilon)-\lambda_{k}^{\prime}(\epsilon)\right) \psi_{k}(\epsilon)
$$

The scalar product with $\psi_{\ell}(\epsilon)$, where $\ell \neq k$, implies the bounded derivatives

$$
\begin{aligned}
\psi_{\ell}^{*} \psi_{k}^{\prime}(\epsilon) & =\frac{\psi_{\ell}^{*}(\epsilon) \breve{B} \psi_{k}(\epsilon)}{\lambda_{k}(\epsilon)-\lambda_{\ell}(\epsilon)} \quad \ell \neq k, \\
\lambda_{k}^{\prime}(\epsilon) & =\psi_{k}(\epsilon)^{*} \breve{B} \psi_{k}(\epsilon) .
\end{aligned}
$$

The remaining component in the $\psi_{k}(\epsilon)$ direction becomes zero by the normalization

$$
0=\frac{\mathrm{d}}{\mathrm{d} \epsilon}\left(\psi_{k}(\epsilon)^{*} \psi_{k}(\epsilon)\right)=2 \operatorname{Re}\left(\left(\frac{\mathrm{d} \psi_{k}(\epsilon)}{\mathrm{d} \epsilon}\right)^{*} \psi_{k}(\epsilon)\right)=2\left(\frac{\mathrm{d} \psi_{k}(\epsilon)}{\mathrm{d} \epsilon}\right)^{*} \psi_{k}(\epsilon) .
$$

In particular, the nonlinear eigenvalue problem (3.8) is in the form of (3.9), based on (3.10); hence, it can be written as the system of $d^{2}+d$ partial differential equations

$$
\begin{aligned}
\frac{\partial}{\partial \epsilon} \Psi_{j k}(x, \epsilon) & =\sum_{\ell \neq k} \Psi_{j \ell}(x, \epsilon) \frac{\left(\nabla \Psi^{*}(x, \epsilon) \cdot \nabla \Psi(x, \epsilon)\right)_{\ell k}}{\lambda_{k}(x, \epsilon)-\lambda_{\ell}(x, \epsilon)}, \quad j, k=1, \ldots, d \\
\frac{\partial}{\partial \epsilon} \lambda_{k}(x, \epsilon) & =\left(\nabla \Psi^{*}(x, \epsilon) \cdot \nabla \Psi(x, \epsilon)\right)_{k k}, \quad k=1, \ldots, d
\end{aligned}
$$

with the initial data

$$
\begin{aligned}
\Psi(x, 0) & =\tilde{\Psi}(x), \\
\lambda_{k}(x, 0) & =\tilde{\lambda}_{k}(x),
\end{aligned}
$$

and $0<\epsilon<\frac{1}{4 M}$. There is a power series solution for large $M$ in the case that $V$ is real analytic and the eigenvalues $\tilde{\lambda}_{k}$ are distinct for all $x$ : since then also $\tilde{\lambda}$ and $\tilde{\Psi}$ are real analytic and the Cauchy-Kovalevskaya theorem, see [5], yields a local solution to the nonlinear system of partial differential equations (3.13).

The assumption of distinct eigenvalues $\lambda_{1}(0)<\lambda_{2}(0)<\cdots<\lambda_{d}(0)$ and the combination of $(3.12),(3.11)$, and

$$
\psi_{k}(\epsilon)-\psi_{k}(0)=\int_{0}^{\epsilon} \psi_{k}^{\prime}(s) \mathrm{d} s
$$

establishes, for sufficiently small $\epsilon$,

$$
\psi_{k}(\epsilon)-\psi_{k}(0)=\mathcal{O}(\epsilon)
$$

and differentiation of (3.11), with respect to the parameter $x \in \mathbb{R}^{N}$, yields

$$
\sup _{|\gamma| \leq m, x \in \mathbb{R}^{N}}\left\|\partial_{x}^{\gamma}\left(\psi_{k}(\epsilon)-\psi_{k}(0)\right)\right\|=\mathcal{O}(\epsilon) .
$$


The induction defined in (3.16) uses this estimate for $\epsilon=1 /(4 M)$ in $(3.15)$ and (3.17).

An alternative to solve (3.8) exactly, which requires less regularity on $V$, is the following approximate solution based on fixed point iterations to obtain an asymptotic expansion. Let $\mathcal{S}(C)$ denote an orthogonal matrix of eigenvectors to a $d \times d$ Hermitian matrix $C$, with the columns in the order of the eigenvalues, so that, e.g., $\mathcal{S}(V(x))=\left[\tilde{\Psi}_{1}(x) \tilde{\Psi}_{2}(x) \ldots \tilde{\Psi}_{d}(x)\right]$ as in (2.11). Let $\Psi[1]=\mathcal{S}(V(x))$ and define

$$
\Psi[j+1]=\mathcal{S}\left(\left(V+\frac{1}{4 M} \Psi[j] \nabla \Psi^{*}[j] \cdot \nabla \Psi[j] \Psi^{*}[j]\right)(x)\right) .
$$

Assume that the eigenvalues $\tilde{\lambda}_{j}$ of $V$ are distinct and $V \in\left[\mathcal{C}^{m}\left(\mathbb{R}^{N}\right)\right]^{d^{2}}$. The regular perturbation theory of real symmetric matrices in (3.11) shows that for sufficiently large $M$ and any $k \leq m$

$$
\max _{|\gamma| \leq k-1}\left\|\partial^{\gamma}(\Psi[2]-\Psi[1])\right\|_{L^{\infty}}=\mathcal{O}_{k}\left(M^{-1}\right),
$$

where $\mathcal{O}_{k}$ denotes an order relation that is allowed to depend on $k$. Then induction in $j$, for $j \leq k$, shows that

$$
\max _{|\gamma| \leq k-j}\left\|\partial^{\gamma}(\Psi[j+1]-\Psi[j])\right\|_{L^{\infty}} \leq C_{k, j} M^{-j}
$$

as follows: we have

$$
\begin{aligned}
V & +\frac{1}{4 M} \Psi[j+1] \nabla \Psi^{*}[j+1] \cdot \nabla \Psi[j+1] \Psi^{*}[j+1] \\
& =V+\frac{1}{4 M} \Psi[j] \nabla \Psi^{*}[j] \cdot \nabla \Psi[j] \Psi^{*}[j]+\mathcal{O}_{1}\left(M^{-(j+1)}\right)
\end{aligned}
$$

so that regular perturbation theory implies that the left-hand side is diagonalized by

$$
\Psi[j+2]=\Psi[j+1]+\mathcal{O}\left(M^{-(j+1)}\right)
$$

and there is a constant $K_{k, j}$ such that

$$
C_{k, j+1} \leq K_{k, j} C_{k, j} .
$$

The choice $\Psi=\Psi[\kappa]$ implies

$$
\begin{aligned}
\bar{H}(x, p)= & \Psi^{*}[\kappa] \# H \# \Psi[\kappa] \\
= & \underbrace{\Psi^{*}[\kappa]\left(\frac{|p|^{2}}{2} \mathrm{I}+V+\frac{1}{4 M} \Psi[\kappa-1] \nabla \Psi^{*}[\kappa-1] \cdot \nabla \Psi[\kappa-1] \Psi^{*}[\kappa-1]\right) \Psi[\kappa]}_{=: \frac{|p|^{2}}{2} \mathrm{I}+\Lambda(x)} \\
& +\frac{1}{4 M} \Psi^{*}[\kappa]\left(\Psi[\kappa] \nabla \Psi^{*}[\kappa] \cdot \nabla \Psi[\kappa] \Psi^{*}[\kappa]\right. \\
= & : \underbrace{\frac{|p|^{2}}{2} \mathrm{I}+\Lambda(\kappa)}_{=: \bar{H}_{0}}+\underbrace{r_{0}}_{\mathcal{O}\left(M^{-\kappa}\right)},
\end{aligned}
$$


where

$$
\Lambda(x)=\tilde{\Lambda}(x)+\mathcal{O}\left(M^{-1}\right)
$$

is diagonal with $\tilde{\Lambda}_{j j}=\tilde{\lambda}_{j}$ as in (2.11) and $r_{0}$ is the term with the factor $\frac{1}{4 M} \Psi^{*}[\kappa](\ldots) \Psi[\kappa]$, which only depends on the $x$-coordinate. Here $\kappa \leq m$, where $V \in\left[\mathcal{C}^{m}\left(\mathbb{R}^{N}\right)\right]^{d^{2}}$ and we remind that

$$
\bar{H}_{0}=\frac{|p|^{2}}{2} \mathrm{I}+\Lambda(x)=\underbrace{\frac{|p|^{2}}{2} \mathrm{I}+\tilde{\Lambda}(x)}_{=\tilde{H}}+\mathcal{O}\left(M^{-1}\right) .
$$

We see that

$$
\partial_{t} \hat{\bar{A}}_{t}=\mathrm{i} M^{1 / 2}\left[\hat{\bar{H}}, \hat{\bar{A}}_{t}\right]=\mathrm{i} M^{1 / 2}\left[\hat{\bar{H}}_{0}, \hat{\bar{A}}_{t}\right]+\mathrm{i} M^{1 / 2}\left[\hat{r}_{0}, \hat{\bar{A}}_{t}\right]
$$

consists of a diagonal part and a small coupling $\mathcal{O}\left(M^{-\kappa+1 / 2}\right)$ part. This asymptotic recursion for $\Psi[j]$ is typically not convergent; therefore, the error term $r_{0}=\mathcal{O}\left(M^{-\kappa}\right)$ may be large if $\kappa$ is large, unless $M$ is very large, which is a reason to avoid large values of $\kappa$.

\subsection{Approximation in Time of Observables}

The transformation to diagonal $\bar{H}$ and $\bar{A}_{0}$ yields restrictions to the set of observables $A_{0}$ that we can analyze. The aim of this section is to describe these restrictions and present the time- dependent molecular dynamics observable that will be used to approximate $\bar{A}_{t}$.

3.2.1. Time-Dependent Molecular Dynamics. The symbol $\breve{A}:[0, \infty) \times \mathbb{R}^{2 N} \rightarrow$ $\mathbb{C}^{d \times d}$ that satisfies

$$
\partial_{t} \breve{A}_{t}=\left\{\bar{H}_{0}, \breve{A}_{t}\right\}, \quad t>0, \breve{A}_{0}=\bar{A}_{0},
$$

approximates $\bar{A}_{t}$, as we shall see in Lemma 3.9. By writing the Poisson bracket in the right-hand side explicitly, we see that Eq. (3.19) is a scalar linear hyperbolic partial differential equation for each component:

$$
\partial_{t} \breve{A}_{j j}(t, x, p)=\left.\left(\nabla_{p} \cdot \nabla_{x^{\prime}}-\nabla_{x} \cdot \nabla_{p^{\prime}}\right)\left(\left(\bar{H}_{0}\right)_{j j}(x, p) \breve{A}_{j j}\left(t, x^{\prime}, p^{\prime}\right)\right)\right|_{\left(x^{\prime}, p^{\prime}\right)=(x, p)} .
$$

This partial differential equation can be solved by the method of characteristics, which generates molecular dynamics paths as follows. Let $\breve{A}_{j j}$ be constant along the characteristic

$$
\breve{A}_{j j}\left(t, z_{0}\right):=\breve{A}_{j j}\left(0, z_{t}^{j}\left(z_{0}\right)\right)
$$

where the characteristic path $z_{t}^{j}=\left(x_{t}, p_{t}\right)$ solves the Hamiltonian system

$$
\begin{aligned}
& \dot{x}_{t}=p_{t} \\
& \dot{p}_{t}=-\nabla \lambda_{j}\left(x_{t}\right), \quad t>0
\end{aligned}
$$


with initial data $\left(x_{0}, p_{0}\right)=z_{0}$ and the Hamiltonian $\left(\bar{H}_{0}(x, p)\right)_{j j}=\left(\frac{|p|^{2}}{2} \mathrm{I}+\right.$ $\Lambda(x))_{j j}=\frac{|p|^{2}}{2}+\lambda_{j}(x)$. For each $j$, we have

$$
\underbrace{\partial_{t} \breve{A}_{j j}\left(0, z_{t}^{j}\left(z_{0}\right)\right)}_{=\left.\partial_{s} \breve{A}_{j j}\left(0, z_{t}^{j}\left(z_{s}\right)\right)\right|_{s=0}}=\left\{\left(\bar{H}_{0}\right)_{j j}\left(z_{0}\right), \breve{A}_{j j}\left(0, z_{t}^{j}\left(z_{0}\right)\right)\right\},
$$

where the equality in the left-hand side holds because the Hamiltonian system is autonomous and the Poisson bracket in the right-hand side is obtained from the chain rule differentiation at $s=0$. We conclude that (3.19) holds for $\breve{A}$ constructed by (3.21).

3.2.2. The Set of Allowed Observables. Our approximation of canonical quantum observables becomes implicit in the following sense. Given a Hamiltonian symbol $H$, we can determine electron states $\Psi$ so that $\bar{H}=\Psi^{*} \# H \# \Psi$ is diagonal. For any diagonal initial symbols $\bar{A}_{0}$ and $\bar{B}_{0}$, we will, for instance, show that the molecular dynamics observable

$$
\int_{\mathbb{R}^{2 N}} \operatorname{Tr}\left(\breve{A}_{\tau}(z) \bar{B}_{0}(z) e^{-\beta \bar{H}(z)}\right) \mathrm{d} z
$$

approximates the quantum observable $\operatorname{Tr}\left(\hat{A}_{\tau} \hat{B}_{0} e^{-\beta \hat{H}}\right)$, where

$$
A_{0}=\Psi \# \bar{A}_{0} \# \Psi^{*} \text { and } B_{0}=\Psi \# \bar{B}_{0} \# \Psi^{*} .
$$

To approximate a given quantum observable

$$
\operatorname{Tr}\left(\hat{A}_{\tau} \hat{B}_{0} e^{-\beta \hat{H}}\right)
$$

is in this formulation possible only if $\bar{A}_{0}=\Psi^{*} \# A_{0} \# \Psi$ and $\bar{B}_{0}=\Psi^{*} \# B_{0} \# \Psi$ are diagonal (or almost diagonal). From a given diagonal $\bar{A}_{0}$, it is therefore direct to determine $A_{0}$ but the opposite to first choose $A_{0}$ then requires to verify whether $\bar{A}_{0}$ is diagonal. The set of such $A_{0}$ is in the special case where $\bar{A}_{0}$ only depends on $x$ given by $A_{0}=\Psi \bar{A}_{0} \Psi^{*}$, with $\bar{A}_{0}$ any diagonal matrix. If in addition $\bar{A}_{0}(x)=a(x) \mathrm{I}$, for any scalar function $a: \mathbb{R}^{N} \rightarrow \mathbb{R}$, we obtain $A_{0}(x)=\bar{A}_{0}(x)=a(x) \mathrm{I}$ and similarly for $B_{0}$.

We note that if eigenvalue surfaces cross, i.e., if $\lambda_{j}(x)=\lambda_{j+1}(x)$ for some $j$ and $x$, then $\nabla \Psi(x)$ may not be in $L^{2}\left(\mathbb{R}^{N}\right)$. We have assumed that the observable symbols $\bar{A}_{0}$ and $\bar{B}_{0}$ are diagonal in the same coordinate transformation $\Psi[\kappa]$ that approximately diagonalizes the Hamiltonian, in the composition way (3.18). Example of observables that cannot be diagonalized by the same transformation as the Hamiltonian are $A_{0}(z)=x \Psi_{\cdot 1}(x)\left(\Psi_{\cdot 2}\right)^{*}(x)$ and $B_{0}(z)=x \Psi_{\cdot 1}(x)\left(\Psi_{\cdot 2}\right)^{*}(x)$ and the correlation based on these observables are then not applicable in Theorems 3.2, 3.6 and 3.7, in contrast to the projections $\Psi_{\cdot j}\left(\Psi_{\cdot j}\right)^{*}$ in Remark 2.3.

\subsection{Assumptions and Theorems}

This section states our main results on molecular dynamics approximation of quantum observables in the canonical ensemble. The molecular dynamics is based on the Hamiltonian system formulated in (3.21-3.23). The observable $\bar{A}_{j j}\left(0, z_{t}^{j}\left(z_{0}\right)\right)$, for each $j \in\{1, \ldots, d\}$, is constant along the molecular 
dynamics path and provides the classical approximation of the corresponding quantum observable as we shall see in Theorems 3.2, 3.6 and 3.7. By assuming that the potential $V$ is real analytic, the nonlinear eigenvalue problem (3.8) generates a matrix $\Psi$ of eigenvectors and eigenvalues $\lambda_{j}$ that yields a diagonal Hamiltonian $\bar{H}=\bar{H}_{0}$ with vanishing remainder $r_{0}=0$, as defined in (3.18). The observables $\hat{A}_{\tau}=\hat{\Psi} \hat{\bar{A}}_{\tau} \hat{\Psi}^{*}$ and $\hat{B}=\hat{\Psi} \hat{\bar{B}}_{0} \hat{\Psi}^{*}$ satisfy

Theorem 3.2. Assume that $V$ is real analytic, $\bar{A}_{0}$ and $\bar{B}_{0}$ are diagonal, the $d \times d$ matrix-valued Hamiltonian $H$ has distinct eigenvalues, and that there is a constant $C$ such that

$$
\begin{aligned}
& \max _{i} \sum_{|\alpha| \leq 3}\left\|\partial_{x}^{\alpha} \partial_{x_{i}} \lambda_{j}\right\|_{L^{\infty}\left(\mathbb{R}^{N}\right)} \leq C, \\
& \sum_{|\alpha| \leq 3}\left\|\partial_{z}^{\alpha} \bar{A}_{j j}(0, \cdot)\right\|_{L^{2}\left(\mathbb{R}^{2 N}\right)} \leq C, \\
&\left\|e^{-\beta \bar{H}(z)}\right\|_{L^{2}\left(\mathbb{R}^{2 N}\right)}+\left\|\bar{B}_{0}(z) e^{-\beta \bar{H}(z)}\right\|_{L^{2}\left(\mathbb{R}^{2 N}\right)} \leq C,
\end{aligned}
$$

hold, then there is a constant $c$, depending on $C$, such that the canonical ensemble average satisfies

$$
\begin{aligned}
& \left|\frac{\operatorname{Tr}\left(\hat{A}_{\tau} \hat{\Psi}\left(\bar{B}_{0} e^{-\beta \bar{H}}\right)^{\hat{\Psi}} \hat{\Psi}^{*}\right)}{\operatorname{Tr}\left(\widehat{\Psi} \widehat{e^{-\beta \bar{H}}} \hat{\Psi^{*}}\right)}-\sum_{j=1}^{d} \int_{\mathbb{R}^{2 N}} \frac{\bar{A}_{j j}\left(0, z_{\tau}^{j}\left(z_{0}\right)\right) \bar{B}_{j j}\left(z_{0}\right) e^{-\beta \bar{H}_{j j}\left(z_{0}\right)}}{\sum_{k=1}^{d} \int_{\mathbb{R}^{2 N}} e^{-\beta \bar{H}_{k k}(z)} \mathrm{d} z} \mathrm{~d} z_{0}\right| \\
& \quad \leq c M^{-1}
\end{aligned}
$$

where $z_{\tau}^{j}$ solves (3.22).

We note that

$$
\frac{\operatorname{Tr}\left(\hat{A}_{\tau} \hat{\Psi}\left(\bar{B}_{0} e^{-\beta \bar{H}}\right) \hat{\Psi}^{*}\right)}{\operatorname{Tr}\left(\hat{\Psi} \widehat{e^{-\beta \bar{H}}} \hat{\Psi}^{*}\right)}=\frac{\operatorname{Tr}\left(\hat{\bar{A}}_{\tau}\left(\bar{B}_{0} e^{-\beta \bar{H}}\right) \hat{)}\right.}{\operatorname{Tr}\left(\widehat{e^{-\beta \bar{H}}}\right)}
$$

and that although $V$ is assumed to be analytic, this assumption is used only to construct $\Psi$ and $\lambda$. For instance, the constant $c$ does not depend on the size of the fourth-order derivatives of $\lambda$. Here we have used the quantization of the classical density, $\widehat{e^{-\beta \bar{H}}}$, as discussed in Sect. 2.2. Our related results for the density $e^{-\beta \hat{H}}$ require similar but more assumptions on the regularity of the data given below. We note that all assumptions are based on a fixed number of derivatives not depending on $N$.

Assumption 3.3. Assume that $\kappa=1$ or $\kappa=2$ in (3.18) defines the remainder $r_{0}$, the eigenvalues $\Lambda$, the Hamiltonian $\bar{H}_{0}$ and that there is a constant $C$ such that 


$$
\begin{aligned}
& \left\|r_{0}\right\|_{L^{\infty}\left(\mathbb{R}^{N}\right)} \leq C M^{-\kappa} \\
& \left\||\nabla \Lambda|^{2}\right\|_{L^{\infty}\left(\mathbb{R}^{N}\right)}+\|\Delta \Lambda\|_{L^{\infty}\left(\mathbb{R}^{N}\right)}+\max _{j, k}\left\|\partial_{x_{j}} \partial_{x_{k}} \Lambda\right\|_{L^{\infty}\left(\mathbb{R}^{N}\right)} \leq C \\
& \sum_{j k} \sup _{t \in[0,1 / T]}\left\|e^{-t \bar{H}_{0}} p_{j} \partial_{p_{k}} \bar{B}_{0}\right\|_{L^{2}\left(\mathbb{R}^{2 N}\right)} \\
& \quad+\sum_{j k} \sup _{t \in[0,1 / T]}\left\|e^{-t \bar{H}_{0}} \partial_{p_{j}} \partial_{p_{k}} \bar{B}_{0}\right\|_{L^{2}\left(\mathbb{R}^{2 N}\right)} \leq C \\
& \sum_{j k} \sup _{t \in[0,1 / T]}\left\|e^{-t \bar{H}_{0}} p_{j} p_{k} \bar{B}_{0}\right\|_{L^{2}\left(\mathbb{R}^{2 N}\right)} \leq C \\
& \sup _{t \in[0,1 / T]}\left\|e^{-t \bar{H}_{0}} \Delta_{x} \bar{B}_{0}\right\|_{L^{2}\left(\mathbb{R}^{2 N}\right)}+\sup _{t \in[0,1 / T]}\left\|e^{-t \bar{H}_{0}} \nabla \Lambda \cdot \nabla_{x} \bar{B}_{0}\right\|_{L^{2}\left(\mathbb{R}^{2 N}\right)} \leq C, \\
& \sup _{t \in[0,1 / T]}\left\|e^{-t \bar{H}_{0}} \bar{B}_{0}\right\|_{L^{2}\left(\mathbb{R}^{2 N}\right)} \leq C, \\
& \quad \sup _{t \in[0,1 / T]}\left\|e^{-t \bar{H}_{0}}\left(p \cdot \nabla_{x} \bar{B}_{0}-\nabla \Lambda \cdot \nabla_{p} \bar{B}_{0}\right)\right\|_{L^{2}\left(\mathbb{R}^{2 N}\right)} \leq C \\
& \left\|\bar{A}_{0}\right\|_{L^{2}\left(\mathbb{R}^{2 N}\right)} \leq C, \\
& \quad \sup _{t \in[0,1 / T]}\left\|e^{-t \bar{H}_{0}}\right\|_{L^{2}\left(\mathbb{R}^{2 N}\right)} \leq C .
\end{aligned}
$$

Assumption 3.4. Assume there is a constant $C$ such that

$$
\begin{aligned}
& \sup _{t \in[0,1 / T]}\left\|e^{-t \bar{H}_{0}}\right\|_{L^{2}\left(\mathbb{R}^{2 N}\right)} \leq C, \\
& \max _{i} \sum_{|\alpha| \leq 3}\left\|\partial_{x}^{\alpha} \partial_{x_{i}} \lambda_{j}\right\|_{L^{\infty}\left(\mathbb{R}^{N}\right)} \leq C, \\
& \sum_{|\alpha| \leq 3}\left\|\partial_{z}^{\alpha} \bar{A}_{j j}(0, \cdot)\right\|_{L^{2}\left(\mathbb{R}^{2 N}\right)} \leq C, \\
& \left\|\bar{B}_{0}(z) e^{-\beta \bar{H}_{0}(z)}\right\|_{L^{2}\left(\mathbb{R}^{2 N}\right)} \leq C .
\end{aligned}
$$

Assumption 3.5. Assume $\kappa=2$ in (3.18) defines the remainder $r_{0}$, the eigenvalues $\Lambda$, the Hamiltonian $\bar{H}_{0}$ and that there is a constant $C$ such that

$$
\begin{aligned}
& \left\|r_{0}\right\|_{L^{\infty}\left(\mathbb{R}^{N}\right)} \leq C M^{-2}, \\
& \frac{1}{(2 \pi)^{2 N}}\left\|\mathcal{F}\left(\partial_{z_{n}} e^{-\bar{H}_{0}}\right)\right\|_{L^{1}\left(\mathbb{R}^{2 N}\right)} \leq C, \\
& \sum_{n}\left\|\partial_{z_{n}}\left(p \cdot \bar{B}_{0}-\nabla \Lambda \cdot \bar{B}_{0}\right)\right\|_{L^{2}\left(\mathbb{R}^{2 N}\right)} \leq C, \\
& \frac{1}{(2 \pi)^{2 N}}\left\|\mathcal{F}\left(p \cdot \bar{B}_{0}-\nabla \Lambda \cdot \bar{B}_{0}\right)\right\|_{L^{1}\left(\mathbb{R}^{2 N}\right)} \leq C .
\end{aligned}
$$

Based on these assumptions, we can formulate two additional theorems for the observables $\hat{A}_{\tau}=\hat{\Psi} \hat{\bar{A}}_{\tau} \hat{\Psi}^{*}$ and $\hat{B}=\hat{\Psi} \hat{\bar{B}}_{0} \hat{\Psi}^{*}$, with $\Psi=\Psi[\kappa], \kappa \in\{1,2\}$, defined in (3.14) and the diagonal Hamiltonian $\tilde{H}=\bar{H}_{0}+\mathcal{O}\left(M^{-1}\right)$ in (2.12). 
Theorem 3.6. Assume that $\bar{A}_{0}$ and $\bar{B}_{0}$ are diagonal, the $d \times d$ matrix-valued Hamiltonian $H$ has distinct eigenvalues, and that Assumptions 3.3 and 3.4 hold, then the canonical ensemble average satisfies

$$
\begin{aligned}
& \frac{\frac{1}{2} \operatorname{Tr}\left(\hat{A}_{\tau}\left(\hat{B}_{0} e^{-\beta \hat{H}}+e^{-\beta \hat{H}} \hat{B}_{0}\right)\right)}{\operatorname{Tr}\left(e^{-\beta \hat{H}}\right)} \\
& \quad=\sum_{j=1}^{d} \int_{\mathbb{R}^{2 N}} q_{j} \bar{A}_{j j}\left(0, z_{\tau}^{j}\left(z_{0}\right)\right) \bar{B}_{j j}\left(z_{0}\right) \frac{e^{-\beta \tilde{H}_{j j}\left(z_{0}\right)}}{\int_{\mathbb{R}^{2 N}} e^{-\beta \tilde{H}_{j j}(z)} \mathrm{d} z} \mathrm{~d} z_{0}+\mathcal{O}\left(M^{-1 / 2}\right),
\end{aligned}
$$

where $z_{\tau}^{j}=\left(x_{\tau}, p_{\tau}\right)$ solves

$$
\begin{aligned}
& \dot{x}_{t}=p_{t} \\
& \dot{p}_{t}=-\nabla \tilde{\lambda}_{j}\left(x_{t}\right), \quad t>0,
\end{aligned}
$$

and $q_{j}=q_{j}(\tilde{H})$ is defined by (2.14). If in addition Assumption 3.5 holds, then the estimate in (3.30) holds with the more accurate bound $\mathcal{O}\left(M^{-1}\right)$ replacing $\mathcal{O}\left(M^{-1 / 2}\right)$.

By comparing instead to the Weyl-quantized classical Gibbs density $\widehat{e^{-\beta \bar{H}_{0}}}$, we have the following more accurate error estimate, that only requires Assumption 3.4.

Theorem 3.7. Assume that $\bar{A}_{0}$ and $\bar{B}_{0}$ are diagonal, the $d \times d$ matrix- valued Hamiltonian $H$ has distinct eigenvalues, that $\Psi=\Psi[2]$ and that the Assumptions 3.4, (3.24) and (3.26) hold, then the canonical ensemble average satisfies

$$
\begin{aligned}
& \frac{\operatorname{Tr}\left(\hat{A}_{\tau} \hat{\Psi}\left(\bar{B}_{0} e^{-\beta \bar{H}_{0}}\right) \hat{\Psi^{*}}\right)}{\operatorname{Tr}\left(\hat{\Psi} e^{-\beta \bar{H}_{0}} \hat{\Psi}^{*}\right)}=\frac{\operatorname{Tr}\left(\hat{\bar{A}}_{\tau}\left(\bar{B}_{0} e^{-\beta \bar{H}_{0}}\right) \hat{)}\right.}{\operatorname{Tr}\left(\widehat{e^{-\beta \bar{H}_{0}}}\right)} \\
& \quad=\sum_{j=1}^{d} \int_{\mathbb{R}^{2 N}} q_{j} \bar{A}_{j j}\left(0, z_{\tau}^{j}\left(z_{0}\right)\right) \bar{B}_{j j}\left(z_{0}\right) \frac{e^{-\beta\left(\bar{H}_{0}\right)_{j j}\left(z_{0}\right)}}{\int_{\mathbb{R}^{2 N}} e^{-\beta\left(\bar{H}_{0}\right)_{j j}(z)} \mathrm{d} z} \mathrm{~d} z_{0}+\mathcal{O}\left(M^{-1}\right),
\end{aligned}
$$

where $z_{\tau}^{j}$ solves $(3.22)$ and $q_{j}=q_{j}\left(\bar{H}_{0}\right)$ is defined by $(2.14)$.

\subsection{Structure of the Proofs}

It is useful to split our estimation into two parts

$$
\begin{aligned}
\operatorname{Tr}( & \left.\hat{\bar{A}}_{\tau} \frac{1}{2}\left(\hat{\bar{B}}_{0} e^{-\beta \hat{\bar{H}}}+e^{-\beta \hat{\bar{H}}} \hat{\bar{B}}_{0}\right)-\widehat{\breve{A}(\tau, z)} \bar{B}_{0} \widehat{e^{-\beta H_{0}}}\right) \\
= & \frac{1}{2} \operatorname{Tr}\left(\hat { \overline { A } } _ { \tau } \left(\hat{\bar{B}}_{0} e^{-\beta \hat{\bar{H}}}+e^{-\beta \hat{\bar{H}}} \hat{\bar{B}}_{0}-\left(\bar{B}_{0} e^{-\beta H_{0}} \hat{)}-\left(e^{-\beta H_{0}} \bar{B}_{0} \hat{)}\right)\right)\right.\right. \\
& +\operatorname{Tr}\left(\left(\hat{\bar{A}}_{\tau}-\widehat{\widehat{A}(\tau, z)}\right)\left(\bar{B}_{0} e^{-\beta H_{0}} \hat{)}\right)\right.
\end{aligned}
$$

where the first part is the approximation error of the Gibbs density operator, which is estimated in Lemma 3.8, and the second part is the approximation error of the dynamics of the observable, which is estimated in Lemma 3.9. 
Theorems 3.7 and 3.2 use only Lemma 3.9, while Theorem 3.6 uses both Lemmas 3.8 and 3.9. A third Lemma 3.10 improves the $\mathcal{O}\left(M^{-1 / 2}\right)$ bound in Lemma 3.8 to $\mathcal{O}\left(M^{-1}\right)$ under additional assumptions.

Lemma 3.8. Assume that the $d \times d$ matrix symbols $\bar{A}_{0}(z)$ and $\bar{B}_{0}(z)$ are diagonal and the bounds in Assumption 3.3 hold, then

$$
\frac{\operatorname{Tr}\left(\hat{\bar{A}}_{\tau}\left(\hat{\bar{B}}_{0} e^{-\beta \hat{\bar{H}}}-\left(\bar{B}_{0} e^{-\beta H_{0}}\right) \hat{)}\right)\right.}{\operatorname{Tr}\left(e^{-\beta \hat{H}}\right)}=\mathcal{O}\left(M^{-\min (1 / 2, \kappa-1 / 2)}\right) .
$$

Lemma 3.9. Assume that the $d \times d$ matrix symbols $\bar{A}_{0}(z)$ and $\bar{B}_{0}(z)$ are diagonal and the bounds in Assumption 3.4 hold, then

$$
\frac{\operatorname{Tr}\left(\left(\hat{\bar{A}}_{\tau}-\widehat{\breve{A}(\tau, z)}\right)\left(\bar{B}_{0} e^{-\beta H_{0}}\right)^{-}\right)}{\operatorname{Tr}\left(e^{-\beta \hat{H}_{0}}\right)}=\mathcal{O}\left(M^{-1}\right) .
$$

Lemma 3.10. Assume that the $d \times d$ matrix symbols $\bar{A}_{0}(z)$ and $\bar{B}_{0}(z)$ are diagonal and the bounds in Assumption 3.5 hold, then

$$
\begin{aligned}
& \frac{\operatorname{Tr}\left(\hat{\bar{A}}_{\tau}\left(\hat{\bar{B}}_{0} e^{-\beta \hat{\bar{H}}}+e^{-\beta \hat{\bar{H}}} \hat{\bar{B}}_{0}-\left(\bar{B}_{0} e^{-\beta H_{0}}\right) \hat{-}-\left(e^{-\beta H_{0}} \bar{B}_{0} \hat{)}\right)\right)\right.}{\operatorname{Tr}\left(e^{-\beta \hat{H}}\right)} \\
& \quad=\mathcal{O}\left(M^{-\min (1, \kappa-1 / 2)}\right) .
\end{aligned}
$$

The results in Lemmas 3.8-3.10 have clear limitations since the error estimate of the approximation of the Gibbs density operator in Lemmas 3.8 and 3.10 is not uniform in $N$ and $T^{-1}$ and the approximation error of the observable dynamics in Lemma 3.9 is not uniform in $\tau$. This means that many particles and low temperature yield a large approximation error of the density operator. The approximation error of the observable dynamics depends exponentially on time, $e^{c \tau}$, but $c$ is uniform in $N$ provided the assumptions in Theorem 3.2 hold uniformly in $N$. In conclusion by combining (2.13), (3.32) and Lemmas 3.83.10, we obtain the theorems.

The three proofs of the lemmas, in Sect. 4, have the same structure with three steps - find an error representation, estimate remainder terms in Moyal expansions and evaluate the trace - described roughly as follows.

3.4.1. Error Representation. In the case of Lemma 3.9, we compare the classical dynamics

$$
\partial_{t} y(t, z)=\left\{\bar{H}_{0}(z), y(t, z)\right\}, \quad t>0, \quad y(0, \cdot)=\bar{A}_{0},
$$

with the quantum dynamics

$$
\widehat{\bar{A}_{t}}=e^{\mathrm{i} t M^{1 / 2} \hat{\bar{H}}} \bar{A}_{0} e^{-\mathrm{i} t M^{1 / 2} \hat{\bar{H}}}=: \widehat{\bar{y}(t, z)},
$$

that satisfies

$$
\partial_{t} \widehat{\bar{y}(t, z)}=\mathrm{i} M^{1 / 2}[\hat{\bar{H}}, \widehat{\bar{y}(t, z)}], \quad t>0, \quad \widehat{\bar{y}(0, \cdot)}=\hat{\bar{A}}_{0} .
$$

The definition of the composition rule (3.5) yields

$$
\partial_{t} \bar{y}(t, z)=\mathrm{i} M^{1 / 2}(\bar{H}(z) \# \bar{y}(t, z)-\bar{y}(t, z) \# \bar{H}(z)), \quad t>0, \quad \bar{y}(0, \cdot)=\bar{A}_{0},
$$


and Duhamel's principle applied to $\widehat{y(t, z)}-\widehat{\bar{y}(t, z)}$ implies the error representation

$$
\begin{aligned}
& \widehat{y(t, z)}-\widehat{\bar{y}(t, z)} \\
& =\int_{0}^{t} e^{\mathrm{i}(t-s) M^{1 / 2} \hat{\bar{H}}}\left(\left\{\bar{H}_{0}(z), y(s, z)\right\}-\mathrm{i} M^{1 / 2}(\bar{H} \# y(s, z)-y(s, z) \# \bar{H}) \hat{)}\right. \\
& \quad \times e^{-\mathrm{i}(t-s) M^{1 / 2} \hat{\bar{H}}} \mathrm{~d} s .
\end{aligned}
$$

For the other two lemmas, related representations are obtained using $e^{-t \hat{\bar{H}}}$ instead of $e^{\mathrm{i} t M^{1 / 2} \hat{\bar{H}}}$.

3.4.2. Estimation of Remainder Terms and Evaluation of the Trace. We will use the composition rule (3.5) to estimate remainder terms in the error representation (3.34). Expansion of the exponential in the composition rule leads to the so-called Moyal expansion. The usual estimates of the remainder terms in Moyal expansions determine the $L^{2}\left(\mathbb{R}^{N}\right)$ operator norm from $L^{\infty}$ norm estimates of order $N$ derivatives of the remainder symbol, using the CalderonVaillancourt theorem, see [18, Theorem 4.23]. To avoid derivatives of high order, if $N$ is large, we instead estimate the remainder terms in the form $\operatorname{Tr}(\hat{R} \hat{C})$, for Hermitian operators $\hat{R}$ and $\hat{C}$ on $L^{2}\left(\mathbb{R}^{N}\right)$, by the $L^{2}$ norms of their symbols. We use the Hilbert-Schmidt inner product, $\operatorname{Tr}\left(\hat{R}^{*} \hat{C}\right)$, and the corresponding Hilbert-Schmidt norm, $\|\hat{R}\|_{\mathcal{H} \mathcal{S}}^{2}=\operatorname{Tr}\left(\hat{R}^{*} \hat{R}\right)=\operatorname{Tr}\left(\hat{R}^{2}\right)$, and Lemma 2.1 as follows

$$
\begin{aligned}
\left|\operatorname{Tr}\left(\hat{R}^{*} \hat{C}\right)\right|^{2} & \leq \operatorname{Tr}\left(\hat{R}^{*} \hat{R}\right) \operatorname{Tr}\left(\hat{C}^{*} \hat{C}\right) \\
& =\left(\frac{\sqrt{M}}{2 \pi}\right)^{2 N} \int_{\mathbb{R}^{2 N}} \operatorname{Tr} R^{2}(z) \mathrm{d} z \int_{\mathbb{R}^{2 N}} \operatorname{Tr} C^{2}(z) \mathrm{d} z .
\end{aligned}
$$

Lemmas 3.11 and 3.12 estimate the $L^{2}\left(\mathbb{R}^{2 N}\right)$ norm of the remainder terms in Moyal compositions by integration by parts, roughly as follows

$$
\begin{aligned}
\int_{\mathbb{R}^{2 N}} & |e^{\frac{\mathrm{i}}{2 M^{1 / 2}}\left(\nabla_{x^{\prime}} \cdot \nabla_{p}-\nabla_{x} \cdot \nabla_{p^{\prime}}\right)} \underbrace{A(x, p) B\left(x^{\prime}, p^{\prime}\right)}_{=: r\left(x, p, x^{\prime}, p^{\prime}\right)}|_{z^{\prime}=z}^{2} \mathrm{~d} z \\
= & \int_{\mathbb{R}^{4 N}}\left(e^{\frac{\mathrm{i}}{2 M^{1 / 2}}\left(\nabla_{x^{\prime}} \cdot \nabla_{p}-\nabla_{x} \cdot \nabla_{p^{\prime}}\right)} r\left(z, z^{\prime}\right)\right)^{*} \\
& \times\left(e^{\frac{\mathrm{i}}{2 M^{1 / 2}}\left(\nabla_{x^{\prime}} \cdot \nabla_{p}-\nabla_{x} \cdot \nabla_{p^{\prime}}\right)} r\left(z, z^{\prime}\right)\right) \delta\left(z-z^{\prime}\right) \mathrm{d} z \mathrm{~d} z^{\prime} \\
= & \int_{\mathbb{R}^{4 N}} r^{*}\left(z, z^{\prime}\right) \\
& \times e^{\frac{-\mathrm{i}}{2 M^{1 / 2}}\left(\nabla_{x^{\prime}} \cdot \nabla_{p}-\nabla_{x} \cdot \nabla_{p^{\prime}}\right)}\left(\left(e^{\frac{\mathrm{i}}{2 M^{1 / 2}}\left(\nabla_{x^{\prime}} \cdot \nabla_{p}-\nabla_{x} \cdot \nabla_{p^{\prime}}\right)} r\left(z, z^{\prime}\right)\right) \delta\left(z-z^{\prime}\right)\right) \mathrm{d} z \mathrm{~d} z^{\prime} \\
= & \cdots \leq \frac{1}{(2 \pi)^{4 N}}\|\mathcal{F} A\|_{L^{1}\left(\mathbb{R}^{2 N}\right)}^{2}\|B\|_{L^{2}\left(\mathbb{R}^{2 N}\right)}^{2},
\end{aligned}
$$

where the last steps indicated by " $=\cdots \leq$ " are explained in the proof of Lemmas 3.11 and 3.12 using the Fourier transform of the Dirac measure $\delta\left(z-z^{\prime}\right)$, 
that $\mathrm{i} \nabla_{x} \cdot \nabla_{p}$ is anti-Hermitian (so that $\left(e^{\frac{\mathrm{i}}{2 M^{1 / 2}} \nabla_{x} \cdot \nabla_{p}}\right)^{*}=e^{-\frac{\mathrm{i}}{2 M^{1 / 2}} \nabla_{x} \cdot \nabla_{p}}$ is unitary) and applying Young's inequality to convolutions of Fourier transforms. Here $\mathcal{F}$ denotes the Fourier transform (2.2). In our proof of Theorems 3.7 and 3.2 and to prove (3.30) in Theorem 3.6, we need this estimate only in the special case where one function depends only on the $x$ coordinate, i.e., $A(x)$, and then the right-hand side becomes $\|A\|_{L^{\infty}\left(\mathbb{R}^{N}\right)}^{2}\|B\|_{L^{2}\left(\mathbb{R}^{2 N}\right)}^{2}$. It is a substantial difference using $\|A\|_{L^{\infty}\left(\mathbb{R}^{N}\right)}^{2}$ since a bound on $\|\mathcal{F} A\|_{L^{1}\left(\mathbb{R}^{2 N}\right)}^{2}$ is related to $2(N+1)$ derivatives of $A$ in $L^{2}\left(\mathbb{R}^{2 N}\right)$, see $(3.37)$.

The special form of the Hamiltonian, namely $H(x, p)=\frac{|p|^{2}}{2} \mathrm{I}+V(x)$, is essential to only obtain the case $\|A(x) \# C(x, p)\|_{L^{2}\left(\mathbb{R}^{2 N}\right)}$ in our analysis for the $\mathcal{O}\left(M^{-1 / 2}\right)$ bound in (3.30), which by Lemma 3.12 is bounded by $\|A\|_{L^{\infty}\left(\mathbb{R}^{N}\right)}\|C\|_{L^{2}\left(\mathbb{R}^{2 N}\right)}$, and not

$$
\|C(x, p) \# D(x, p)\|_{L^{2}\left(\mathbb{R}^{2 N}\right)} \leq \frac{1}{(2 \pi)^{2 N}}\|\mathcal{F} C\|_{L^{1}\left(\mathbb{R}^{2 N}\right)}\|D\|_{L^{2}\left(\mathbb{R}^{2 N}\right)}
$$

from (3.40). An $L^{1}$-bound on the Fourier transform of a function, which is required in (3.28) and (3.29) to obtain the accuracy $\mathcal{O}\left(M^{-1}\right)$ in $(3.30)$, is more demanding on regularity than the $L^{\infty}$-norm of the function. For instance, we have

$$
\begin{aligned}
\int_{\mathbb{R}^{2 N}}|\mathcal{F} g(\xi)| \mathrm{d} \xi & =\int_{\mathbb{R}^{2 N}}\left(1+|\xi|^{2}\right)^{(N+1)}|\mathcal{F} g(\xi)|\left(1+|\xi|^{2}\right)^{-(N+1)} \mathrm{d} \xi \\
& \leq\left\|\left(1+|\xi|^{2}\right)^{(N+1)} \mathcal{F} g(\xi)\right\|_{L^{2}\left(\mathbb{R}^{2 N}\right)}\left\|\left(1+|\xi|^{2}\right)^{-(N+1)}\right\|_{L^{2}\left(\mathbb{R}^{2 N}\right)} \\
& \leq \text { const. }\left\|(1+\Delta)^{(N+1)} g\right\|_{L^{2}\left(\mathbb{R}^{2 N}\right)}
\end{aligned}
$$

The eigenvalue functions $\tilde{\lambda}_{j}(x)$ and their Laplacian are typically proportional to the number of particles, since the Hamiltonian is the energy of the system. Therefore, the corresponding estimates in the first row of (3.25) are bounded by a constant proportional to $N$, while (3.27) can be uniform with respect to $N$. Also the remainder term $r_{0}$, related to $4 M^{-1} \nabla \Psi^{*} \cdot \nabla \Psi$ in (3.18), may be proportional to $N$. Therefore, also the estimate $\left\|\lambda_{j}-\tilde{\lambda}_{j}\right\|_{L^{\infty}\left(\mathbb{R}^{N}\right)}=$ $\mathcal{O}\left(M^{-1}\right)$, obtained from (3.18) with $\kappa=1$, may have a constant proportional to $N$.

\subsection{Remainder Terms in the Moyal Composition}

The following two lemmas estimate remainder terms in the Moyal expansions of the compositions that we will use below. Here $\mathcal{F}[C]$ denotes the standard Fourier transform of $C$, see 2.2. We also use the abbreviation $z \equiv(x, p) \in \mathbb{R}^{2 N}$ and $\zeta \equiv\left(\xi_{x}, \xi_{p}\right) \in \mathbb{R}^{2 N}$ in which case we write $\mathcal{F}[C](\zeta)=\int_{\mathbb{R}^{2 N}} C\left(z^{\prime}\right) e^{-\mathrm{i} z^{\prime} \cdot \zeta} \mathrm{d} z^{\prime}$.

Lemma 3.11. Assume $C: \mathbb{R}^{2 N} \rightarrow \mathbb{C}^{d \times d}$ and $D: \mathbb{R}^{2 N} \rightarrow \mathbb{C}^{d \times d}$ and that there exist constants $M_{\gamma}, N_{\gamma}$ such that for integer multi-indices $\gamma=\left(\gamma_{1}, \ldots, \gamma_{2 N}\right)$

$$
\begin{aligned}
\left\|\partial_{z}^{\gamma} C\right\|_{L^{2}\left(\mathbb{R}^{2 N}\right)}+\left\|\partial_{z}^{\gamma} D\right\|_{L^{2}\left(\mathbb{R}^{2 N}\right)} & \leq M_{\gamma}, \quad \text { for all }|\gamma| \leq m+1, \\
\left\|\mathcal{F}\left[\partial_{z}^{\gamma} C\right]\right\|_{L^{1}\left(\mathbb{R}^{2 N}\right)} \leq N_{\gamma}, & \text { for all }|\gamma| \leq m+1,
\end{aligned}
$$


then the composition has the expansion

$$
C \# D(x, p)=\left.\sum_{n=0}^{m} \frac{1}{n !}\left(\mathrm{i} \frac{\nabla_{x^{\prime}} \cdot \nabla_{p}-\nabla_{x} \cdot \nabla_{p^{\prime}}}{2 M^{1 / 2}}\right)^{n} C(x, p) D\left(x^{\prime}, p^{\prime}\right)\right|_{(x, p)=\left(x^{\prime}, p^{\prime}\right)}+r(z),
$$

where the remainder $r \in L^{2}\left(\mathbb{R}^{2 N}\right)$ satisfies

$$
\begin{aligned}
\|r\|_{L^{2}\left(\mathbb{R}^{2 N}\right)}^{2} \leq & \left(\frac{1}{4 M}\right)^{m+1} \frac{1}{(m !)^{2}(2 m+1)} \\
& \times \| \int_{0}^{1} e^{\frac{i s}{M^{1 / 2}}\left(\nabla_{x^{\prime}} \cdot \nabla_{p}-\nabla_{x} \cdot \nabla_{p^{\prime}}\right)} \\
& \times\left.\left(\nabla_{x^{\prime}} \cdot \nabla_{p}-\nabla_{x} \cdot \nabla_{p^{\prime}}\right)^{m+1} C(x, p) D\left(x^{\prime}, p^{\prime}\right) \mathrm{d} s\right|_{z=z^{\prime}} \|_{L^{2}\left(\mathbb{R}^{2 N}\right)}^{2} \\
= & \mathcal{O}\left(M^{-(m+1)}\right) .
\end{aligned}
$$

If $C(x, p)=A(x)$ depends only on the $x$-coordinate and

$$
\left\|\partial_{x}^{\gamma} A\right\|_{L^{\infty}\left(\mathbb{R}^{N}\right)} \leq M_{\gamma}, \text { for all }|\gamma| \leq m+1
$$

then

$$
\begin{aligned}
r(x, p)= & \left(\frac{1}{2 M^{1 / 2}}\right)^{m+1} \\
& \times\left.\int_{0}^{1} e^{-\frac{\mathrm{i} s}{2} M^{-1 / 2} \nabla_{x} \cdot \nabla_{p}}\left(-\mathrm{i} \nabla_{x} \cdot \nabla_{p}\right)^{m+1} A(x) D\left(x^{\prime}, p\right) \frac{(1-s)^{m}}{m !} \mathrm{d} s\right|_{x^{\prime}=x},
\end{aligned}
$$

or if $C(x, p)=A(p)$ depends only on the $p$-coordinate and $\left\|\partial_{p}^{\gamma} A\right\|_{L^{\infty}\left(\mathbb{R}^{N}\right)} \leq$ $M_{\gamma}$, for all $|\gamma| \leq m+1$ then

$$
\begin{aligned}
r(x, p)= & \left(\frac{1}{2 M^{1 / 2}}\right)^{m+1} \\
& \times\left.\int_{0}^{1} e^{\frac{\mathrm{i} s}{2} M^{-1 / 2} \nabla_{x} \cdot \nabla_{p}}\left(\mathrm{i} \nabla_{x} \cdot \nabla_{p}\right)^{m+1} A(p) D\left(x, p^{\prime}\right) \frac{(1-s)^{m}}{m !} \mathrm{d} s\right|_{p^{\prime}=p} .
\end{aligned}
$$

Lemma 3.12. Assume $C: \mathbb{R}^{2 N} \rightarrow \mathbb{C}^{d \times d}$ and $D: \mathbb{R}^{2 N} \rightarrow \mathbb{C}^{d \times d}$ belong to $L^{2}\left(\mathbb{R}^{2 N}\right)$ and in addition one of these functions has its Fourier transform in $L^{1}\left(\mathbb{R}^{2 N}\right)$, then

$$
\begin{aligned}
& \|C \# D\|_{L^{2}\left(\mathbb{R}^{2 N}\right)} \\
& \quad \leq \frac{1}{(2 \pi)^{2 N}} \min \left(\|C\|_{L^{2}\left(\mathbb{R}^{2 N}\right)}\|\mathcal{F} D\|_{L^{1}\left(\mathbb{R}^{2 N}\right)},\|\mathcal{F} C\|_{L^{1}\left(\mathbb{R}^{2 N}\right)}\|D\|_{L^{2}\left(\mathbb{R}^{2 N}\right)}\right),
\end{aligned}
$$

and if $A: \mathbb{R}^{N} \rightarrow \mathbb{C}^{d \times d}$ depends only on the $x$-coordinate (or only on the $p$ coordinate) and is bounded in $L^{\infty}\left(\mathbb{R}^{N}\right)$ then

$$
\begin{aligned}
\|A \# D\|_{L^{2}\left(\mathbb{R}^{2 N}\right)} & \leq\|A\|_{L^{\infty}\left(\mathbb{R}^{N}\right)}\|D\|_{L^{2}\left(\mathbb{R}^{N}\right)}, \\
\|D \# A\|_{L^{2}\left(\mathbb{R}^{2 N}\right)} & \leq\|A\|_{L^{\infty}\left(\mathbb{R}^{N}\right)}\|D\|_{L^{2}\left(\mathbb{R}^{N}\right)} .
\end{aligned}
$$

The proofs of Lemmas 3.11 and 3.12 are in Sect. 4.4. 


\section{Proofs}

\subsection{Proof of Lemma 3.8}

Proof. The proof has three steps: error representations, estimation of remainder terms and evaluation of the trace.

Step 1. Error representations Let $y:[0, \infty) \times \mathbb{R}^{2 N} \rightarrow \mathbb{C}^{d \times d}$ and $\bar{y}_{i}:[0, \infty) \times$ $\mathbb{R}^{2 N} \rightarrow \mathbb{C}^{d \times d}, i=0,1,2$ be defined by

$$
\begin{aligned}
y(t, z) & =e^{-t \bar{H}_{0}(z)} \bar{B}_{0}, \\
\widehat{y_{0}(t, z)} & =e^{-t \hat{\bar{H}}}, \\
\widehat{\bar{y}_{1}(t, z)} & =\hat{\bar{B}}_{0} e^{-t \hat{\bar{H}}}, \\
\widehat{\bar{y}_{2}(t, z)} & =e^{-t \hat{\bar{H}}} \hat{\bar{B}}_{0} .
\end{aligned}
$$

Differentiation yields the linear ordinary differential equation, with $z$ as a parameter,

$$
\partial_{t} y(t, z)=-\bar{H}_{0}(z) y(t, z), \quad t>0, \quad y(0, \cdot)=\bar{B}_{0} .
$$

The dynamics of $\bar{y}_{1}$ satisfies

$$
\partial_{t} \widehat{\bar{y}_{1}(t, z)}=\hat{\bar{B}}_{0} \frac{d}{d t} e^{-t \hat{\bar{H}}}=-\hat{\bar{B}}_{0} e^{-t \hat{\bar{H}}} \hat{\bar{H}}=-\widehat{\bar{y}_{1}(t, z)} \hat{\bar{H}}
$$

and the corresponding evolution equation for $\bar{y}_{1}$ is the linear partial differential equation

$$
\partial_{t} \bar{y}_{1}(t, z)=-\bar{y}_{1}(t, z) \# \bar{H}(z), \quad t>0, \quad \bar{y}_{1}(0, \cdot)=\bar{B}_{0},
$$

with time-independent generator. Analogously we obtain the equations

$$
\begin{array}{lll}
\partial_{t} \bar{y}_{2}(t, z)=-\bar{H}(z) \# \bar{y}_{2}(t, z), & t>0, & \bar{y}_{2}(0, \cdot)=\bar{B}_{0}, \\
\partial_{t} \bar{y}_{0}(t, z)=-\bar{H}(z) \# \bar{y}_{0}(t, z), & t>0, & \bar{y}_{0}(0, \cdot)=\mathrm{I} .
\end{array}
$$

We have the two linear equations

$$
\begin{aligned}
\partial_{t} \widehat{\bar{y}_{1}(t, z)}+\bar{y}_{1}(t \widehat{, z) \# \bar{H}}(z)=0, \quad t>0, \\
\partial_{t} \widehat{y(t, z)}+y\left(t \widehat{, z) \# \bar{H}}(z)=y\left(t, \widehat{z) \# \bar{H}}(z)-y\left(t, \widehat{z) \bar{H}_{0}}(z), \quad t>0,\right.\right.\right.
\end{aligned}
$$

and Duhamel's principle implies

$$
\begin{aligned}
\hat{y}(t, z)-\widehat{y_{1}(t, z)} & =\int_{0}^{t}\left(y(s, z) \# \bar{H}(z)-y(s, z) \bar{H}_{0}(z) \hat{)} \bar{y}_{0} \widehat{(t-s}, z\right) \mathrm{d} s \\
& =\int_{0}^{t} \underbrace{\left(y(s, z) \# \bar{H}(z)-y(s, z) \bar{H}_{0}(z)\right)}_{=: \hat{R}_{1 s}} e^{-(t-s) \hat{\bar{H}}} \mathrm{~d} s .
\end{aligned}
$$

Similarly, we have

$$
\begin{aligned}
& \partial_{t} \widehat{\bar{y}_{2}(t, z)}+\bar{H}(z) \widehat{\# \bar{y}_{2}}(t, z)=0, \quad t>0, \\
& \partial_{t} \widehat{y(t, z)}+\bar{H}(\widehat{z) \# y(} t, z)=\bar{H}(\widehat{z) \# y(t}, z)-y\left(t \widehat{, z) \bar{H}_{0}}(z), \quad t>0,\right.
\end{aligned}
$$


and

$$
\begin{aligned}
& \left.\hat{y}(t, z)-\widehat{\bar{y}_{2}(t, z)}=\int_{0}^{t} \bar{y}_{0} \widehat{(t-s}, z\right)\left(\bar{H}(z) \# y(s, z)-y(s, z) \bar{H}_{0}(z) \hat{)} \mathrm{d} s\right. \\
& =\int_{0}^{t} e^{-(t-s) \hat{H}} \underbrace{\left(\bar{H}(z) \# y(s, z)-y(s, z) \bar{H}_{0}(z)\right)}_{=: \hat{R}_{2 s}} \mathrm{~d} s .
\end{aligned}
$$

The remainder terms satisfy by (3.5)

$$
\begin{aligned}
R_{1 s}= & \left(e^{-s \bar{H}_{0}} \bar{B}_{0}\right) \# \bar{H}-e^{-s \bar{H}_{0}} \bar{B}_{0} \bar{H}_{0} \\
= & \left(\left(e^{-s \bar{H}_{0}} \bar{B}_{0}\right) \# \bar{H}_{0}-e^{-s \bar{H}_{0}} \bar{B}_{0} \bar{H}_{0}\right)+\left(e^{-s \bar{H}_{0}} \bar{B}_{0}\right) \# r_{0} \\
= & \left(-\frac{i M^{-1 / 2}}{2}\left\{e^{-s \bar{H}_{0}} \bar{B}_{0}, \bar{H}_{0}\right\}+r_{2}\right)+\left(e^{-s \bar{H}_{0}} \bar{B}_{0}\right) \# r_{0} \\
= & (-\frac{\mathrm{i}}{2 M^{1 / 2}} \bar{B}_{0} \underbrace{\left\{e^{-s \bar{H}_{0}}, \bar{H}_{0}\right\}}_{=0}-\frac{\mathrm{i}}{2 M^{1 / 2}} e^{-s \bar{H}_{0}}\left\{\bar{B}_{0}, \bar{H}_{0}\right\}+r_{2}) \\
& \left.+\left(e^{-s \bar{H}_{0}} \bar{B}_{0}\right) \# r_{0}\right) \\
= & \left(-\frac{\mathrm{i}}{2 M^{1 / 2}} e^{-s \bar{H}_{0}}\left\{\bar{B}_{0}, \bar{H}_{0}\right\}+r_{2}\right)+\left(e^{-s \bar{H}_{0}} \bar{B}_{0}\right) \# r_{0}
\end{aligned}
$$

and analogously

$$
\begin{aligned}
R_{2 s} & =\left(\bar{H} \# e^{-s \bar{H}_{0}} \bar{B}_{0}\right)-e^{-s \bar{H}_{0}} \bar{B}_{0} \bar{H}_{0} \\
& =\left(\bar{H}_{0} \# e^{-s \bar{H}_{0}} \bar{B}_{0}\right)-e^{-s \bar{H}_{0}} \bar{B}_{0} \bar{H}_{0}+r_{0} \#\left(e^{-s \bar{H}_{0}} \bar{B}_{0}\right) \\
& =\left(-\frac{\mathrm{i}}{2 M^{1 / 2}}\left\{\bar{H}_{0}, e^{-s \bar{H}_{0}} \bar{B}_{0}\right\}+r_{2}^{\prime}\right)+r_{0} \#\left(e^{-s \bar{H}_{0}} \bar{B}_{0}\right) \\
& =\left(-\frac{\mathrm{i}}{2 M^{1 / 2}} e^{-s \bar{H}_{0}}\left\{\bar{H}_{0}, \bar{B}_{0}\right\}+r_{2}^{\prime}\right)+r_{0} \#\left(e^{-s \bar{H}_{0}} \bar{B}_{0}\right) .
\end{aligned}
$$

We have $r_{2}=\left(r_{2}^{\prime}\right)^{*}$ and the next step shows that

$$
\left\|r_{2}\right\|_{L^{2}\left(\mathbb{R}^{2 N}\right)}=\mathcal{O}\left(M^{-1}\right)
$$

Step 2. Estimation of remainder terms The remainder representations (3.38) and (3.39) applied to the $x$ and $p$ dependent terms in $\bar{H}_{0}$ separately imply

$$
\begin{aligned}
& r_{2}(x, p, s) \\
& =\frac{-1}{4 M} \int_{0}^{1} e^{\frac{\mathrm{i} \sigma}{2 M^{1 / 2}}\left(\nabla_{x} \cdot \nabla_{p}\right)}\left(\left(\nabla_{x} \cdot \nabla_{p}\right)^{2}\left(\frac{|p|^{2}}{2} \mathrm{I} e^{-s \bar{H}_{0}\left(x, p^{\prime}\right)} \bar{B}_{0}\left(x, p^{\prime}\right)\right)\right. \\
& \left.\quad+\left(\nabla_{x} \cdot \nabla_{p}\right)^{2}\left(\Lambda(x) e^{-s \bar{H}_{0}\left(x^{\prime}, p\right)} \bar{B}_{0}\left(x^{\prime}, p\right)\right)\right)\left.\right|_{\substack{x=x^{\prime} \\
p=p^{\prime}}}(1-\sigma) \mathrm{d} \sigma
\end{aligned}
$$


which can be written

$$
\begin{aligned}
& r_{2}(x, p, s) \\
&=\frac{-1}{4 M} \int_{0}^{1} e^{\frac{\mathrm{i} \sigma}{2 M^{1 / 2}}\left(\nabla_{x} \cdot \nabla_{p}\right)}\left(e^{-s \bar{H}_{0}\left(x, p^{\prime}\right)} \bar{B}_{0}\left(x, p^{\prime}\right)\left(s^{2} \nabla \Lambda(x) \cdot \nabla \Lambda(x)-s \Delta \Lambda(x)\right)\right. \\
&-2 s e^{-s \bar{H}_{0}\left(x, p^{\prime}\right)} \nabla \Lambda(x) \cdot \nabla_{x} \bar{B}_{0}\left(x, p^{\prime}\right)+e^{-s \bar{H}_{0}\left(x, p^{\prime}\right)} \Delta_{x} \bar{B}_{0}\left(x, p^{\prime}\right) \\
&-s e^{-s \bar{H}_{0}\left(x^{\prime}, p\right)} \bar{B}_{0}\left(x^{\prime}, p\right) \Delta \Lambda(x) \\
&+s^{2} e^{-s \bar{H}_{0}\left(x^{\prime}, p\right)} \bar{B}_{0}\left(x^{\prime}, p\right) \sum_{j k} p_{j} p_{k} \partial_{x_{j}} \partial_{x_{k}} \Lambda(x) \\
&-2 s e^{-s \bar{H}_{0}\left(x^{\prime}, p\right)} \sum_{j k} p_{j} \partial_{x_{j}} \partial_{x_{k}} \Lambda(x) \partial_{p_{k}} \bar{B}_{0}\left(x^{\prime}, p\right) \\
&\left.+e^{-s \bar{H}_{0}\left(x^{\prime}, p\right)} \sum_{j k} \partial_{x_{j}} \partial_{x_{k}} \Lambda(x) \partial_{p_{k}} \partial_{p_{k}} \bar{B}_{0}\left(x^{\prime}, p\right)\right)_{x=x^{\prime}, p=p^{\prime}}(1-\sigma) \mathrm{d} \sigma .
\end{aligned}
$$

We have by (3.41) in Lemma 3.12, where $A$ is the function of $x$ and $D$ is the function of $\left(x^{\prime}, p^{\prime}\right)$ in the estimates of $r_{0}$ and $r_{2}$ in (3.18) and (4.5),

$$
\begin{aligned}
& \left\|r_{0} \# e^{-s \bar{H}_{0}} \bar{B}_{0}\right\|_{L^{2}\left(\mathbb{R}^{2 N}\right)} \leq\left\|r_{0}\right\|_{L^{\infty}\left(\mathbb{R}^{N}\right)}\left\|e^{-s \bar{H}_{0}} \bar{B}_{0}\right\|_{L^{2}\left(\mathbb{R}^{2 N}\right)}, \\
& \frac{\left\|e^{-s \bar{H}_{0}}\left\{\bar{B}_{0}, \bar{H}_{0}\right\}\right\|_{L^{2}\left(\mathbb{R}^{2 N}\right)}}{M^{1 / 2}}=\frac{\left\|e^{-s \bar{H}_{0}}\left(p \cdot \nabla_{x} \bar{B}_{0}-\nabla \Lambda \cdot \nabla_{p} \bar{B}_{0}\right)\right\|_{L^{2}\left(\mathbb{R}^{2 N}\right)}}{M^{1 / 2}}, \\
& 4 M\left\|r_{2}\right\|_{L^{2}\left(\mathbb{R}^{2 N}\right)} \leq s\left\|e^{-s \bar{H}_{0}} \bar{B}_{0}(x, p)\right\|_{L^{2}\left(\mathbb{R}^{2 N}\right)}\|\Delta \Lambda\|_{L^{\infty}\left(\mathbb{R}^{2 N}\right)} \\
& +s^{2} \sum_{j k}\left\|p_{j} p_{k} e^{-s \bar{H}_{0}} \bar{B}_{0}(x, p)\right\|_{L^{2}\left(\mathbb{R}^{2 N}\right)}\left\|\partial_{x_{j} x_{k}} \Lambda\right\|_{L^{\infty}\left(\mathbb{R}^{2 N}\right)} \\
& +2 s \sum_{j k}\left\|p_{j} e^{-s \bar{H}_{0}} \partial_{p_{k}} \bar{B}_{0}(x, p)\right\|_{L^{2}\left(\mathbb{R}^{2 N}\right)}\left\|\partial_{x_{j} x_{k}} \Lambda\right\|_{L^{\infty}\left(\mathbb{R}^{2 N}\right)} \\
& +\sum_{j k}\left\|e^{-s \bar{H}_{0}} \partial_{p_{j} p_{k}} \bar{B}_{0}(x, p)\right\|_{L^{2}\left(\mathbb{R}^{2 N}\right)}\left\|\partial_{x_{j} x_{k}} \Lambda\right\|_{L^{\infty}\left(\mathbb{R}^{2 N}\right)} \\
& +\left\|e^{-s \bar{H}_{0}} \bar{B}_{0}(x, p)\left(s^{2} \nabla \Lambda \cdot \nabla \Lambda-s \Delta \Lambda\right)\right\|_{L^{2}\left(\mathbb{R}^{2 N}\right)} \\
& +2 s\left\|e^{-s \bar{H}_{0}} \nabla \Lambda \cdot \nabla_{x} \bar{B}_{0}(x, p)\right\|_{L^{2}\left(\mathbb{R}^{2 N}\right)} \\
& +\left\|e^{-s \bar{H}_{0}} \Delta_{x} \bar{B}_{0}(x, p)\right\|_{L^{2}\left(\mathbb{R}^{2 N}\right)} \text {. }
\end{aligned}
$$

Here we see that $r_{0}$ depends only on the $x$-coordinate and the composition in $r_{2}$ has one factor that also depends only on the $x$-coordinate. Therefore, by Lemma 3.11 and Eq. (3.41) we obtain

$$
\left\|R_{1 s}\right\|_{L^{2}\left(\mathbb{R}^{2 N}\right)}+\left\|R_{2 s}\right\|_{L^{2}\left(\mathbb{R}^{2 N}\right)}=\mathcal{O}\left(M^{-1 / 2}\right),
$$

provided there holds

$$
\left\|r_{0}\right\|_{L^{\infty}\left(\mathbb{R}^{N}\right)}=\mathcal{O}\left(M^{-1}\right),
$$


and

$$
\begin{aligned}
& \left\||\nabla \Lambda|^{2}\right\|_{L^{\infty}\left(\mathbb{R}^{N}\right)}+\|\Delta \Lambda\|_{L^{\infty}\left(\mathbb{R}^{N}\right)}+\max _{j, k}\left\|\partial_{x_{j}} \partial_{x_{k}} \Lambda\right\|_{L^{\infty}\left(\mathbb{R}^{N}\right)}=\mathcal{O}(1), \\
& \sum_{j k} \sup _{t \in[0,1 / T]}\left\|e^{-t \bar{H}_{0}} p_{j} \partial_{p_{k}} \bar{B}_{0}\right\|_{L^{2}\left(\mathbb{R}^{2 N}\right)} \\
& \quad+\sum_{j k} \sup _{t \in[0,1 / T]}\left\|e^{-t \bar{H}_{0}} \partial_{p_{j}} \partial_{p_{k}} \bar{B}_{0}\right\|_{L^{2}\left(\mathbb{R}^{2 N}\right)}=\mathcal{O}(1), \\
& \sum_{j k} \sup _{t \in[0,1 / T]}\left\|e^{-t \bar{H}_{0}} p_{j} p_{k} \bar{B}_{0}\right\|_{L^{2}\left(\mathbb{R}^{2 N}\right)}=\mathcal{O}(1), \\
& \sup _{t \in[0,1 / T]}\left\|e^{-t \bar{H}_{0}} \Delta_{x} \bar{B}_{0}\right\|_{L^{2}\left(\mathbb{R}^{2 N}\right)}+\sup _{t \in[0,1 / T]}\left\|e^{-t \bar{H}_{0}} \nabla \Lambda \cdot \nabla_{x} \bar{B}_{0}\right\|_{L^{2}\left(\mathbb{R}^{2 N}\right)}=\mathcal{O}(1), \\
& \sup _{t \in[0,1 / T]}\left\|e^{-t \bar{H}_{0}} \bar{B}_{0}\right\|_{L^{2}\left(\mathbb{R}^{2 N}\right)}=\mathcal{O}(1), \\
& \sup _{t \in[0,1 / T]}\left\|e^{-t \bar{H}_{0}}\left(p \cdot \nabla_{x} \bar{B}_{0}-\nabla \Lambda \cdot \nabla_{p} \bar{B}_{0}\right)\right\|_{L^{2}\left(\mathbb{R}^{2 N}\right)}=\mathcal{O}(1) .
\end{aligned}
$$

Step 3. Evaluation of the trace The Hilbert-Schmidt inner product, $\operatorname{Tr}\left(\hat{B}^{*} \hat{C}\right)$, for symmetric operators on $L^{2}\left(\mathbb{R}^{N}\right)$, and its Cauchy's inequality imply together with (4.1)

$$
\begin{aligned}
{[\operatorname{Tr}} & \left.\left(\hat{\bar{A}}_{\tau}(\hat{\bar{y}}(t)-\hat{y}(t))\right)\right]^{2} \\
& =\left(\operatorname{Tr}\left(\hat{\bar{A}}_{\tau} \int_{0}^{t} \hat{R}_{1 s} e^{-(t-s) \hat{\bar{H}}} \mathrm{~d} s\right)\right)^{2} \\
& =\left(\int_{0}^{t} \operatorname{Tr}\left(\hat{\bar{A}}_{\tau} \hat{R}_{1 s} e^{-(t-s) \hat{\bar{H}}}\right) \mathrm{d} s\right)^{2} \\
& \leq\left(\int_{0}^{t}\left(\operatorname{Tr}\left(e^{\mathrm{i} \tau M^{1 / 2}} \hat{\bar{H}} \hat{\bar{A}}_{0}^{2} e^{-\mathrm{i} \tau M^{1 / 2} \hat{\bar{H}}}\right) \operatorname{Tr}\left(e^{-(t-s) \hat{\bar{H}}} \hat{R}_{1 s}^{2} e^{-(t-s) \hat{\bar{H}}}\right)\right)^{1 / 2} \mathrm{~d} s\right)^{2} \\
& \left.=\left(\int_{0}^{t}\left(\operatorname{Tr}\left(\hat{\bar{A}}_{0}^{2}\right) \operatorname{Tr}\left(\hat{R}_{1 s}^{2} e^{-2(t-s) \hat{\bar{H}}}\right)\right)^{1 / 2} \mathrm{~d} s\right)^{2}\right) \mathrm{d} s \\
& \left.\leq \int_{0}^{t} \operatorname{Tr}\left(\hat{\bar{A}}_{0}^{2}\right) \mathrm{d} s \int_{0}^{t} \operatorname{Tr}(\underbrace{\left(\hat{\Psi}_{\hat{R}_{1 s}} \hat{\Psi}^{*}\right.}_{=: \hat{\bar{R}}_{s}})^{2} e^{-2(t-s) \hat{H}}\right)
\end{aligned}
$$

Lemma 2.1 establishes

$$
\int_{0}^{t} \operatorname{Tr}\left(\hat{\bar{A}}_{0}^{2}\right) \mathrm{d} s=t\left(\frac{\sqrt{M}}{2 \pi}\right)^{N} \int_{\mathbb{R}^{2 N}} \operatorname{Tr}\left(\bar{A}^{2}(0, z)\right) \mathrm{d} z
$$

and we assume that the initial data satisfies

$$
\left\|\bar{A}_{0}\right\|_{L^{2}\left(\mathbb{R}^{2 N}\right)}=\mathcal{O}(1)
$$


The basis $\left\{\Phi_{n}\right\}_{n=1}^{\infty}$ of solutions to the Schrödinger equation (2.1) implies

$$
\begin{aligned}
\operatorname{Tr}\left(\hat{\bar{R}}_{s}^{2} e^{-2(t-s) \hat{H}}\right) & =\sum_{n=1}^{\infty}\left\langle\Phi_{n}, \hat{\bar{R}}_{s}^{2} e^{-2(t-s) \hat{H}} \Phi_{n}\right\rangle \\
& =\sum_{n=1}^{\infty}\left\langle\Phi_{n}, \hat{\bar{R}}_{s}^{2} \Phi_{n}\right\rangle e^{-2(t-s) E_{n}} \\
& \leq e^{-2(t-s) E_{1}} \sum_{n=1}^{\infty}\left\langle\Phi_{n}, \hat{\bar{R}}_{s}^{2} \Phi_{n}\right\rangle \\
& =e^{-2(t-s) E_{1}} \operatorname{Tr}\left(\hat{R}_{s}^{*} \hat{R}_{s}\right)
\end{aligned}
$$

and Lemmas 2.1, 3.11 and (3.41) combined with (4.6) show that

$$
\begin{aligned}
\operatorname{Tr}\left(\hat{R}_{s}^{*} \hat{R}_{s}\right) & =\left(\frac{\sqrt{M}}{2 \pi}\right)^{N} \int_{\mathbb{R}^{2 N}} \operatorname{Tr}\left(R_{s}^{*} R_{s}\right) \mathrm{d} z \\
& =\mathcal{O}\left(M^{-1+N / 2}\right),
\end{aligned}
$$

so that

$$
\operatorname{Tr}\left(\hat{\bar{A}}_{\tau}(\hat{\bar{y}}(t)-\hat{y}(t))\right)=\mathcal{O}\left(M^{-1 / 2+N / 2}\right) .
$$

In the special case where $\bar{A}_{0}=\bar{B}_{0}=\mathrm{I}$, we similarly obtain

$$
\operatorname{Tr}\left(e^{-\beta \hat{H}}-\widehat{e^{-\beta \bar{H}_{0}}}\right)=\mathcal{O}\left(M^{-1 / 2+N / 2}\right)
$$

provided

$$
\sup _{t \in[0,1 / T]}\left\|e^{-t \bar{H}_{0}}\right\|_{L^{2}\left(\mathbb{R}^{2 N}\right)}=\mathcal{O}(1)
$$

and by (2.7)

$$
\begin{aligned}
& \operatorname{Tr}\left(\widehat{e^{-\beta \bar{H}_{0}}}\right)=\left(\frac{\sqrt{M}}{2 \pi}\right)^{N} \int_{\mathbb{R}^{2 N}} \operatorname{Tr}\left(e^{-\beta \bar{H}_{0}(z)}\right) \mathrm{d} z, \\
& \operatorname{Tr}\left(e^{-\beta \hat{\bar{H}}}\right)=\left(\frac{\sqrt{M}}{2 \pi}\right)^{N}\left(\int_{\mathbb{R}^{2 N}} \operatorname{Tr}\left(e^{-\beta \bar{H}_{0}(z)}\right) \mathrm{d} z+\mathcal{O}\left(M^{-1 / 2}\right)\right) .
\end{aligned}
$$

In conclusion, we have for $M$ sufficiently large

$$
\left|\frac{\operatorname{Tr}\left(\hat{\bar{A}}_{\tau}\left(\left(\bar{B}_{0} e^{-\beta \bar{H}_{0}}\right)^{\hat{n}}-\hat{\bar{B}}_{0} e^{-\beta \hat{\bar{H}}}\right)\right)}{\operatorname{Tr}\left(e^{-\beta \hat{H}}\right)}\right|=\mathcal{O}\left(M^{-1 / 2}\right) .
$$

\subsection{Proof of Lemma 3.9}

Proof. This proof is analogous to the proof of Lemma 3.8 and has three similar steps: error representation, estimation of remainder terms and evaluation of the trace. 
Step 1. Error representation We will compare the classical dynamics $\widehat{y\left(t, z_{0}\right)}:=\widehat{A}\left(t, z_{0}\right)$ with the quantum dynamics

$$
\widehat{\bar{A}_{t}}=e^{\mathrm{i} t M^{1 / 2} \hat{\bar{H}}} \bar{A}_{0} e^{-\mathrm{i} t M^{1 / 2} \hat{\bar{H}}}=: \widehat{\bar{y}(t, z)} .
$$

The classical dynamics for the symbol $y:[0, \infty) \times \mathbb{R}^{2 N} \rightarrow \mathbb{C}^{d \times d}$ satisfies by (3.19) and (3.21) the linear partial differential equation

$$
\partial_{t} y(t, z)=\left\{\bar{H}_{0}(z), y(t, z)\right\}, \quad t>0, \quad y(0, \cdot)=\bar{A}_{0},
$$

that is, we have the diagonal matrix

$$
y_{j k}\left(t, z_{0}\right)=\left\{\begin{array}{cl}
\left(\bar{A}_{0}\right)_{j j}\left(z_{t}^{j}\left(z_{0}\right)\right) & \text { if } k=j, \\
0 & \text { if } k \neq j,
\end{array} \quad \text { for } z_{0} \in \mathbb{R}^{2 N} \text { and } t \geq 0\right. \text {. }
$$

The evolution of $\bar{y}:[0, \infty) \times \mathbb{R}^{2 N} \rightarrow \mathbb{C}^{d \times d}$ is defined by the quantum dynamics

$$
\partial_{t} \widehat{\bar{y}(t, z)}=\mathrm{i} M^{1 / 2}[\hat{\bar{H}}, \widehat{\bar{y}(t, z)}], \quad t>0, \quad \widehat{\bar{y}(0, \cdot)}=\hat{\bar{A}}_{0},
$$

which implies

$$
\partial_{t} \bar{y}(t, z)=\mathrm{i} M^{1 / 2}(\bar{H}(z) \# \bar{y}(t, z)-\bar{y}(t, z) \# \bar{H}(z)), \quad t>0, \quad \bar{y}(0, \cdot)=\bar{A}_{0} .
$$

Duhamel's principle yields

$$
\begin{aligned}
& \widehat{y(t, z)}-\widehat{\bar{y}(t, z)} \\
&=\int_{0}^{t} e^{\mathrm{i}(t-s) M^{1 / 2} \hat{\bar{H}}}\left(\left\{\bar{H}_{0}(z), y(s, z)\right\}-\mathrm{i} M^{1 / 2}(\bar{H} \# y(s, z)-y(s, z) \# \bar{H}) \hat{)}\right. \\
& \times e^{-\mathrm{i}(t-s) M^{1 / 2} \hat{\bar{H}}} \mathrm{~d} s \\
&=: \int_{0}^{t} e^{\mathrm{i}(t-s) M^{1 / 2} \hat{\bar{H}}} \hat{R}_{s} e^{-\mathrm{i}(t-s) M^{1 / 2} \hat{\bar{H}}} \mathrm{~d} s .
\end{aligned}
$$

Step 2. Estimation of remainder terms Since $y(s, z)$ and $\bar{H}_{0}(z)$ are diagonal, the expansion of the composition in Lemma 3.11, and Eq. (3.41) imply

$$
\begin{aligned}
R_{s} & =\left\{\bar{H}_{0}, y(s, \cdot)\right\}-\mathrm{i} M^{1 / 2}(\bar{H} \# y(s, \cdot)-y(s, \cdot) \# \bar{H}) \\
& =\underbrace{\left\{\bar{H}_{0}, y\right\}-\mathrm{i} M^{1 / 2}\left(\bar{H}_{0} \# y-y \# \bar{H}_{0}\right)}_{=r_{2}=\mathcal{O}\left(M^{-1}\right)}-\mathrm{i} M^{1 / 2}\left(r_{0} \# y-y \# r_{0}\right) \\
& =r_{2}-\mathrm{i} M^{1 / 2}\left(r_{0} \# y-y \# r_{0}\right)=\mathcal{O}\left(M^{-\min (1, \kappa-1 / 2)}\right),
\end{aligned}
$$

where by Lemma 3.11

$$
r_{2}=\left.\frac{\mathrm{i}}{16 M} \int_{0}^{1} e^{\mathrm{i} \frac{s}{2 M^{1 / 2}} \nabla_{x} \cdot \nabla_{p}} \sum_{|\alpha|=3} \partial_{x}^{\alpha} \Lambda(x) \partial_{p}^{\alpha} y\left(x^{\prime}, p\right)\right|_{x^{\prime}=x}(1-s)^{2} \mathrm{~d} s,
$$

and by (3.41)

$$
\begin{aligned}
& \left\|r_{2}\right\|_{L^{2}\left(\mathbb{R}^{2 N}\right)} \leq \frac{1}{48 M} \sum_{|\alpha|=3}\left\|\partial_{x}^{\alpha} \Lambda\right\|_{L^{\infty}\left(\mathbb{R}^{N}\right)}\left\|\partial_{p}^{\alpha} y(t, \cdot)\right\|_{L^{2}\left(\mathbb{R}^{2 N}\right)}, \\
& \left\|r_{0} \# y-y \# r_{0}\right\|_{L^{2}\left(\mathbb{R}^{2 N}\right)} \leq 2\left\|r_{0}\right\|_{L^{\infty}\left(\mathbb{R}^{N}\right)}\|y(t, \cdot)\|_{L^{2}\left(\mathbb{R}^{2 N}\right)} .
\end{aligned}
$$


Let $z(t)=z_{t}^{j}$. To estimate $\sum_{|\alpha| \leq 3}\left\|\partial_{p}^{\alpha} y(t, \cdot)\right\|_{L^{2}\left(\mathbb{R}^{2 N}\right)}$ we use the first-order flow $\nabla_{z_{0}} z_{t}^{j}\left(z_{0}\right)=: z^{\prime}(t)$, second- order flow $z_{, k m}^{\prime \prime}(t)=\partial_{z_{k}(0) z_{m}(0)} z(t)$ and thirdorder flow $z^{\prime \prime \prime}(t)$, which are solutions to the system

$$
\begin{aligned}
\dot{z}_{i}(t)= & \left(J \nabla \bar{H}_{0}\left(z_{t}\right)\right)_{i}=: f_{i}\left(z_{t}\right), \\
z_{i, k}^{\prime}(t)= & \mathrm{I}_{i k}+\int_{0}^{t} \sum_{k^{\prime}} f_{i, k^{\prime}}^{\prime}\left(z_{s}\right) z_{k^{\prime}, k}^{\prime}(s) \mathrm{d} s, \quad f_{i, k^{\prime}}^{\prime}(z):=\partial_{z_{k^{\prime}}} f_{i}(z), \\
z_{i, k m}^{\prime \prime}(t)= & \int_{0}^{t}\left(\sum_{k^{\prime}} f_{i, k^{\prime}}^{\prime}\left(z_{s}\right) z_{k^{\prime}, k m}^{\prime \prime}(s)+\sum_{k^{\prime} m^{\prime}} f_{i, k^{\prime} m^{\prime}}^{\prime \prime}\left(z_{s}\right) z_{k^{\prime}, k}^{\prime}(s) z_{m^{\prime}, m}^{\prime}(s)\right) \mathrm{d} s, \\
& f_{i, k^{\prime} m^{\prime}}^{\prime \prime}(z):=\partial_{z_{k^{\prime}} z_{m^{\prime}}} f_{i}(z), \\
z_{i, k m n}^{\prime \prime \prime}(t)= & \int_{0}^{t}\left(\sum_{k^{\prime}} f_{i, k^{\prime}}^{\prime}\left(z_{s}\right) z_{k^{\prime}, k m n}^{\prime \prime \prime}(s)+\sum_{k^{\prime} m^{\prime}} f_{i, k^{\prime} m^{\prime}}^{\prime \prime}\left(z_{s}\right) z_{k^{\prime}, k}^{\prime}(s) z_{m^{\prime}, m n}^{\prime \prime}(s)\right. \\
& +\sum_{k^{\prime} m^{\prime}} f_{i, k^{\prime} m^{\prime}}^{\prime \prime}\left(z_{s}\right) z_{k^{\prime}, k n}^{\prime \prime}(s) z_{m^{\prime}, m}^{\prime}(s) \\
& +\sum_{k^{\prime} n^{\prime}} f_{i, k^{\prime} n^{\prime}}^{\prime \prime}\left(z_{s}\right) z_{k^{\prime}, k m}^{\prime \prime}(s) z_{n^{\prime}, n}^{\prime}(s) \\
& \left.+\sum_{k^{\prime} m^{\prime} n^{\prime}} f_{i, k^{\prime} m^{\prime} n^{\prime}}^{\prime \prime \prime}\left(z_{s}\right) z_{k^{\prime}, k}^{\prime}(s) z_{m^{\prime}, m}^{\prime}(s) z_{n^{\prime}, n}^{\prime}(s)\right) \mathrm{d} s
\end{aligned}
$$

where $J$ is the $2 N \times 2 N$ matrix

$$
J=\left[\begin{array}{cc}
0 & \mathrm{I} \\
-\mathrm{I} & 0
\end{array}\right] .
$$

By summation and maximization over indices, we obtain the integral inequalities

$$
\begin{aligned}
\max _{i k}\left|z_{i, k}^{\prime}(t)\right| \leq & 1+\int_{0}^{t} \sum_{k^{\prime}}\left|f_{i, k^{\prime}}^{\prime}\left(z_{s}\right)\right| \max _{i k}\left|z_{i, k}^{\prime}(s)\right| \mathrm{d} s \\
\max _{i k} \sum_{m}\left|z_{i, k m}^{\prime \prime}(t)\right| \leq & \int_{0}^{t} \sum_{k^{\prime}}\left|f_{i, k^{\prime}}^{\prime}\left(z_{s}\right)\right| \max _{i k} \sum_{m}\left|z_{i, k m}^{\prime \prime}(s)\right| \mathrm{d} s \\
& +\int_{0}^{t} \sum_{k^{\prime} m^{\prime}}\left|f_{i, k^{\prime} m^{\prime}}^{\prime \prime}\left(z_{s}\right)\right|\left(\max _{i k}\left|z_{i, k}^{\prime}(s)\right|\right)^{2} \mathrm{~d} s, \\
\max _{i k} \sum_{m n}\left|z_{i, k m n}^{\prime \prime \prime}(t)\right| \leq & \int_{0}^{t} \sum_{k^{\prime}}\left|f_{i, k^{\prime}}^{\prime}\left(z_{s}\right)\right| \max _{i k} \sum_{m n}\left|z_{i, k m n}^{\prime \prime \prime}(s)\right| \mathrm{d} s \\
& +\int_{0}^{t} \sum_{k^{\prime} m^{\prime}}\left|f_{i, k^{\prime} m^{\prime}}^{\prime \prime}\left(z_{s}\right)\right| \max _{i k}\left|z_{i, k}^{\prime}(s)\right| \max _{i k} \sum_{m}\left|z_{i, k m}^{\prime \prime}(s)\right| \mathrm{d} s \\
& +\int_{0}^{t} \sum_{k^{\prime} m^{\prime} n^{\prime}}\left|f_{i, k^{\prime} m^{\prime} n^{\prime}}^{\prime \prime \prime}\left(z_{s}\right)\right|\left(\max _{i k}\left|z_{i, k}^{\prime}(s)\right|\right)^{3} \mathrm{~d} s .
\end{aligned}
$$


The functions $\max _{i j} \sum_{|\alpha| \leq 2} \partial_{z_{0}}^{\alpha} \partial_{z_{j}} z_{i}\left(t, z_{0}\right)$ can therefore be estimated as in [7] by Gronwall's inequality, which states: if there is a positive constant $K$ and continuous positive functions $\beta, u:[0, \infty) \rightarrow[0, \infty)$ such that

$$
u(t) \leq K+\int_{0}^{t} \beta(s) u(s) \mathrm{d} s, \quad \text { for } t>0,
$$

then

$$
u(t) \leq K e^{\int_{0}^{t} \beta(s) \mathrm{d} s}, \quad \text { for } t>0 .
$$

Gronwall's inequality applied to (4.17) implies

$$
\max _{i j} \sum_{|\alpha| \leq 2}\left\|\partial_{z_{0}}^{\alpha} \partial_{z_{j}} z_{i}\left(t, z_{0}\right)\right\|_{L^{\infty}\left(\mathbb{R}^{2 N}\right)}=\mathcal{O}(1)
$$

provided that

$$
\max _{i} \sum_{|\alpha| \leq 3}\left\|\partial_{x}^{\alpha} \partial_{x_{i}} \lambda_{j}\right\|_{L^{\infty}\left(\mathbb{R}^{N}\right)}=\mathcal{O}(1) .
$$

The flows $z^{\prime}, z^{\prime \prime}, z^{\prime \prime \prime}$ determine the derivatives of the diagonal matrix $y(t, \cdot)$, using $a_{j}(z):=\bar{A}_{j j}(0, z)$, by

$$
\begin{aligned}
\partial_{z_{k}} y_{j j}(t)= & \sum_{k^{\prime}} a_{j, k^{\prime}}^{\prime}\left(z_{t}\right) z_{k^{\prime}, k}^{\prime}(t), \quad a_{j, k^{\prime}}^{\prime}(z):=\partial_{z_{k^{\prime}}} a_{j}(z) \\
\partial_{z_{k} z_{m}} y_{j j}(t)= & \sum_{k^{\prime}} a_{j, k^{\prime}}^{\prime}\left(z_{t}\right) z_{k^{\prime}, k m}^{\prime \prime}(t)+\sum_{k^{\prime} m^{\prime}} a_{j, k^{\prime} m^{\prime}}^{\prime \prime}\left(z_{t}\right) z_{k^{\prime}, k}^{\prime}(t) z_{m^{\prime}, m}^{\prime}(t) \\
\partial_{z_{k} z_{m} z_{n}} y_{j j}(t)= & \sum_{k^{\prime}} a_{j, k^{\prime}}^{\prime}\left(z_{t}\right) z_{k^{\prime}, k m n}^{\prime \prime \prime}(t)+\sum_{k^{\prime} m^{\prime}} a_{j, k^{\prime} m^{\prime}}^{\prime \prime}\left(z_{t}\right) z_{k^{\prime}, k}^{\prime}(t) z_{m^{\prime}, m n}^{\prime \prime}(t) \\
& +\sum_{k^{\prime} m^{\prime}} a_{j, k^{\prime} m^{\prime}}^{\prime \prime}\left(z_{t}\right) z_{k^{\prime}, k n}^{\prime \prime}(t) z_{m^{\prime}, m}^{\prime}(t) \\
& +\sum_{k^{\prime} n^{\prime}} a_{j, k^{\prime} n^{\prime}}^{\prime \prime}\left(z_{t}\right) z_{k^{\prime}, k m}^{\prime \prime}(t) z_{n^{\prime}, n}^{\prime}(t) \\
& +\sum_{k^{\prime} m^{\prime} n^{\prime}} a_{j, k^{\prime} m^{\prime} n^{\prime}}^{\prime \prime \prime}\left(z_{t}\right) z_{k^{\prime}, k}^{\prime}(t) z_{m^{\prime}, m}^{\prime}(t) z_{n^{\prime}, n}^{\prime}(t)
\end{aligned}
$$

The constant in the right-hand side of (4.18) grows typically exponentially with respect to $t$, i.e.,

$$
\max _{i j} \sum_{|\alpha| \leq 2}\left\|\partial_{z_{0}}^{\alpha} \partial_{z_{j}} z_{i}\left(t, z_{0}\right)\right\|_{L^{\infty}\left(\mathbb{R}^{2 N}\right)} \leq e^{c t},
$$

where $c$ is the positive constant in the right-hand side of (4.19). The integration with respect to the initial data measure $\mathrm{d} z_{0}$ can be replaced by integration with respect to $\mathrm{d} z_{t}$ since the phase-space volume is preserved, i.e., the Jacobian determinant

$$
\left|\operatorname{det}\left(\frac{\partial z_{0}^{j}}{\partial z_{t}^{j}}\right)\right|=1
$$


is constant for all time, so that

$$
\begin{aligned}
\int_{\mathbb{R}^{2 N}}\left|\partial_{z_{t}^{j}}^{\alpha} a_{j}\left(0, z_{t}^{j}\left(x_{0}, p_{0}\right)\right)\right|^{2} \mathrm{~d} z_{0} & =\int_{\mathbb{R}^{2 N}}\left|\partial_{z_{t}^{j}}^{\alpha} \bar{A}_{j j}\left(0, z_{t}^{j}\left(x_{0}, p_{0}\right)\right)\right|^{2} \mathrm{~d} z_{0} \\
& =\int_{\mathbb{R}^{2 N}}\left|\partial_{z_{t}^{j}}^{\alpha} \bar{A}_{j j}\left(0, z_{t}^{j}\left(x_{0}, p_{0}\right)\right)\right|^{2}\left|\operatorname{det}\left(\frac{\partial z_{0}^{j}}{\partial z_{t}^{j}}\right)\right| \mathrm{d} z_{t}^{j} \\
& =\int_{\mathbb{R}^{2 N}}\left|\partial_{z_{t}^{j}}^{\alpha} \bar{A}_{j j}\left(0, z_{t}^{j}\right)\right|^{2} \mathrm{~d} z_{t}^{j} .
\end{aligned}
$$

Equations (4.20) and (4.18) therefore imply

$$
\begin{aligned}
& \sum_{|\alpha|=1} \sqrt{\int_{\mathbb{R}^{2 N}}\left|\partial_{p_{0}}^{\alpha} \bar{A}_{j j}\left(0, z_{t}^{j}\left(x_{0}, p_{0}\right)\right)\right|^{2} \mathrm{~d} z_{0}} \\
& \leq \max _{i k}\left\|\partial_{z_{k}(0)} z_{i}(t)\right\|_{L^{\infty}\left(\mathbb{R}^{2 N}\right)} \sum_{|\alpha|=1}\left\|\partial_{z}^{\alpha} \bar{A}_{j j}(0, z)\right\|_{L^{2}\left(\mathbb{R}^{2 N}\right)}, \\
& \sum_{|\alpha|=2} \sqrt{\int_{\mathbb{R}^{2 N}}\left|\partial_{p_{0}}^{\alpha} \bar{A}_{j j}\left(0, z_{t}^{j}\left(x_{0}, p_{0}\right)\right)\right|^{2} \mathrm{~d} z_{0}} \\
& \leq \sum_{n} \max _{i k}\left\|\partial_{z_{n}(0) z_{k}(0)} z_{i}(t)\right\|_{L^{\infty}\left(\mathbb{R}^{2 N}\right)} \sum_{|\alpha|=1}\left\|\partial_{z}^{\alpha} \bar{A}_{j j}(0, z)\right\|_{L^{2}\left(\mathbb{R}^{2 N}\right)} \\
& +\max _{i k}\left\|\partial_{z_{k}(0)} z_{i}(t)\right\|_{L^{\infty}\left(\mathbb{R}^{2 N}\right)}^{2} \sum_{|\alpha|=2}\left\|\partial_{z}^{\alpha} \bar{A}_{j j}(0, z)\right\|_{L^{2}\left(\mathbb{R}^{2 N}\right)},
\end{aligned}
$$

and

$$
\begin{aligned}
\sum_{|\alpha|=3} & \sqrt{\int_{\mathbb{R}^{2 N}}\left|\partial_{p_{0}}^{\alpha} \bar{A}_{j j}\left(0, z_{t}^{j}\left(x_{0}, p_{0}\right)\right)\right|^{2} \mathrm{~d} z_{0}} \\
\leq & \sum_{m n} \max _{i k}\left\|\partial_{z_{m}(0) z_{n}(0) z_{k}(0)} z_{i}(t)\right\|_{L^{\infty}\left(\mathbb{R}^{2 N}\right)} \sum_{|\alpha|=1}\left\|\partial_{z}^{\alpha} \bar{A}_{j j}(0, z)\right\|_{L^{2}\left(\mathbb{R}^{2 N}\right)} \\
& +\sum_{n} \max _{i k}\left\|\partial_{z_{n}(0) z_{k}(0)} z_{i}(t)\right\|_{L^{\infty}\left(\mathbb{R}^{2 N}\right)} \max _{i k}\left\|\partial_{z_{k}(0)} z_{i}(t)\right\|_{L^{\infty}\left(\mathbb{R}^{2 N}\right)}^{2} \\
& \times \sum_{|\alpha|=2}\left\|\partial_{z}^{\alpha} \bar{A}_{j j}(0, z)\right\|_{L^{2}\left(\mathbb{R}^{2 N}\right)} \\
& +\max _{i k}\left\|\partial_{z_{k}(0)} z_{i}(t)\right\|_{L^{\infty}\left(\mathbb{R}^{2 N}\right)}^{3} \sum_{|\alpha|=3}\left\|\partial_{z}^{\alpha} \bar{A}_{j j}(0, z)\right\|_{L^{2}\left(\mathbb{R}^{2 N}\right)}
\end{aligned}
$$

Estimate (4.18) implies that these right-hand sides are bounded, provided that

$$
\begin{aligned}
\max _{i} \sum_{|\alpha| \leq 3}\left\|\partial_{x}^{\alpha} \partial_{x_{i}} \lambda_{j}\right\|_{L^{\infty}\left(\mathbb{R}^{N}\right)} & =\mathcal{O}(1) \\
\sum_{|\alpha| \leq 3}\left\|\partial_{z}^{\alpha} \bar{A}_{j j}(0, \cdot)\right\|_{L^{2}\left(\mathbb{R}^{2 N}\right)} & =\mathcal{O}(1)
\end{aligned}
$$


We note that the assumption on $\lambda_{j}$ is compatible with the assumption that $\tilde{\lambda}_{j}(x)$ tends to infinity as $|x| \rightarrow \infty$, which is imposed to have a discrete spectrum of $\hat{H}$. The choice $\Psi=\Psi[2]$ yields $\lambda_{j}=\tilde{\lambda}_{j}+\mathcal{O}\left(M^{-1}\right)$. The eigenvalues $\lambda_{j}$ have four bounded derivatives if the eigenvalues $\tilde{\lambda}_{j}$ of the potential $V$ are distinct and also the corresponding eigenvectors $\Psi[1]$ and $\Psi[2]$ have four bounded derivatives, which requires the fifth-order derivatives of the potential $V$ to be bounded.

Step 3. Evaluation of the trace Define the diagonal matrix

$$
C(z)=\bar{B}_{0}(z) e^{-\beta \bar{H}_{0}(z)} .
$$

Duhamel's representation implies

$$
\begin{aligned}
\mid \operatorname{Tr} & (\hat{C}(\widehat{\bar{y}(t, z)}-\widehat{y(t)})) \mid \\
= & \operatorname{Tr}\left(\int_{0}^{t} \hat{C} e^{\mathrm{i}(t-s) M^{1 / 2} \hat{\bar{H}}} \hat{R}_{s} e^{-\mathrm{i}(t-s) M^{1 / 2} \hat{\bar{H}}} \mathrm{~d} s\right) \mid \\
& =\left|\int_{0}^{t} \operatorname{Tr}\left(\hat{C} e^{\mathrm{i}(t-s) M^{1 / 2} \hat{\bar{H}}} \hat{R}_{s} e^{-\mathrm{i}(t-s) M^{1 / 2} \hat{\bar{H}}}\right) \mathrm{d} s\right| \\
& =\left|\int_{0}^{t} \operatorname{Tr}\left(e^{-\mathrm{i}(t-s) M^{1 / 2} \hat{\bar{H}}} \hat{C} e^{\mathrm{i}(t-s) M^{1 / 2} \hat{\bar{H}}} \hat{R}_{s}\right) \mathrm{d} s\right|
\end{aligned}
$$

and Cauchy's inequality for the trace, and Lemma 2.1 establishes

$$
\begin{aligned}
\mid \operatorname{Tr} & (\hat{C}(\widehat{\bar{y}(t, z)}-\widehat{y(t)})) \mid \\
& \leq \int_{0}^{t}\left(\operatorname{Tr}\left(e^{-\mathrm{i}(t-s) M^{1 / 2} \hat{H}} \hat{C}^{*} \hat{C} e^{\mathrm{i}(t-s) M^{1 / 2} \hat{\bar{H}}}\right) \operatorname{Tr}\left(\hat{R}_{s}^{*} \hat{R}_{s}\right)\right)^{1 / 2} \mathrm{~d} s \\
& =\int_{0}^{t}\left(\operatorname{Tr}\left(\hat{C}^{*} \hat{C}\right) \operatorname{Tr}\left(\hat{R}_{s}^{*} \hat{R}_{s}\right)\right)^{1 / 2} \mathrm{~d} s \\
& =\left(\frac{\sqrt{M}}{2 \pi}\right)^{N} \int_{0}^{t}\left(\int_{\mathbb{R}^{2 N}} \operatorname{Tr}\left(C^{*} C\right) \mathrm{d} z \int_{\mathbb{R}^{2 N}} \operatorname{Tr}\left(R_{s}^{*} R_{s}\right) \mathrm{dz}\right)^{1 / 2} \mathrm{~d} s \\
& =\left(\frac{\sqrt{M}}{2 \pi}\right)^{N} \int_{0}^{t}\left\|\bar{B}_{0}(z) e^{-\beta \bar{H}_{0}(z)}\right\|_{L^{2}\left(\mathbb{R}^{2 N}\right)}\left\|R_{s}\right\|_{L^{2}\left(\mathbb{R}^{2 N}\right)} \mathrm{d} s,
\end{aligned}
$$

which together with (4.13) prove the lemma provided also

$$
\left\|\bar{B}_{0}(z) e^{-\beta \bar{H}_{0}(z)}\right\|_{L^{2}\left(\mathbb{R}^{2 N}\right)}=\mathcal{O}(1) .
$$

\subsection{Proof of Lemma 3.10}

Proof. The improved bound (3.33) is based on

$$
\left.\left.\widehat{y(t, \cdot)}-\frac{1}{2}\left(\widehat{\bar{y}_{1}(t, \cdot)}+\widehat{\bar{y}_{2}(t, \cdot)}\right)=\frac{1}{2} \int_{0}^{t}\left(\widehat{R_{1 s}} \bar{y}_{0} \widehat{(t-s}, \cdot\right)+\bar{y}_{0} \widehat{(t-s}, \cdot\right) \widehat{R_{2 s}}\right) \mathrm{d} s,
$$


which is obtained by (4.1) and (4.2). Let $r_{1}:=M^{-1 / 2}\left\{\bar{B}_{0}, \bar{H}_{0}\right\} e^{-s \bar{H}_{0}}$. Estimate (4.3) shows that the dominating term in $R_{1 s}$ is $r_{1}$ and similarly by (4.4) the dominating term in $R_{2 s}$ is $-r_{1}$. By (4.1), applied with $\bar{B}_{0}=\mathrm{I}$, we obtain

$$
\hat{\bar{y}}_{0}(s)=\hat{y}(s)+\int_{0}^{s} \hat{R}_{1}\left(s^{\prime}\right) \hat{\bar{y}}_{0}\left(s-s^{\prime}\right) \mathrm{d} s^{\prime},
$$

which replaces the remainder term $\hat{r}_{1} \hat{\bar{y}}_{0}$ included in (4.1) by the smaller term

$$
\left(r_{1} \# y-y \# r_{1}\right) \hat{,}
$$

present in (4.23) and generates the new remainder terms

$$
\begin{aligned}
\int_{0}^{t} \hat{r}_{1} \hat{R}_{1} \hat{\bar{y}}_{0} \mathrm{~d} s & =\int_{0}^{t}\left(r_{1} \# R_{1}\right) \hat{\bar{y}} \hat{\bar{y}}_{0} \mathrm{~d} s \\
\int_{0}^{t} \hat{\bar{y}}_{0} \hat{R}_{1} \hat{r}_{1} \mathrm{~d} s & =\int_{0}^{t} \hat{\bar{y}}_{0}\left(R_{1} \# r_{1}\right) \mathrm{d} s
\end{aligned}
$$

Lemma 3.11 and Eq. (3.40) imply $\left\|r_{1} \# y-y \# r_{1}\right\|_{L^{2}\left(\mathbb{R}^{2 N}\right)}=\mathcal{O}\left(M^{-1}\right)$ provided

$$
\begin{aligned}
\frac{1}{(2 \pi)^{2 N}}\left\|\mathcal{F}\left(\partial_{z_{n}} e^{-\bar{H}_{0}}\right)\right\|_{L^{1}\left(\mathbb{R}^{2 N}\right)} & =\mathcal{O}(1), \\
\sum_{n}\left\|\partial_{z_{n}}\left(p \cdot \bar{B}_{0}-\nabla \Lambda \cdot \bar{B}_{0}\right)\right\|_{L^{2}\left(\mathbb{R}^{2 N}\right)} & =\mathcal{O}(1),
\end{aligned}
$$

and there holds $\left\|r_{1} \# R_{1}\right\|_{L^{2}\left(\mathbb{R}^{2 N}\right)}+\left\|R_{1} \# r_{1}\right\|_{L^{2}\left(\mathbb{R}^{2 N}\right)}=\mathcal{O}\left(M^{-1}\right)$ if

$$
\frac{1}{(2 \pi)^{2 N}}\left\|\mathcal{F}\left(p \cdot \bar{B}_{0}-\nabla \Lambda \cdot \bar{B}_{0}\right)\right\|_{L^{1}\left(\mathbb{R}^{2 N}\right)}=\mathcal{O}(1)
$$

and (4.6) are satisfied. The new error terms

$$
\begin{aligned}
& \operatorname{Tr}\left(\hat{\bar{A}}_{\tau} \int_{0}^{t}\left(r_{1}(s) \# y(t-s)-y(t-s) \# r_{1}(s)\right) \mathrm{d} s\right), \\
& \operatorname{Tr}\left(\hat{\bar{A}}_{\tau} \int_{0}^{t}\left(r_{1} \# R_{1}\right)_{s} \hat{\bar{y}}_{0}(t-s) \mathrm{d} s\right),
\end{aligned}
$$

can then be estimated as in (4.9)-(4.13). We obtain that the first term in the right-hand side of (3.32) has the bound $\mathcal{O}\left(M^{-1}\right)$, by assuming (4.7), (4.8), (4.10), (4.12), (4.24) and (4.25).

\subsection{Weyl Quantization Estimates: Proof of Lemmas 3.11 and 3.12}

The purpose of this section is to prove Lemmas 3.11 and 3.12 that estimate the remainder terms $r_{i}, i=0,2$ in the $L^{2}\left(\mathbb{R}^{2 N}\right)$ norm, and avoid derivatives of high order $N$, using that the Hilbert-Schmidt norm of operators can be bounded based on the $L^{2}\left(\mathbb{R}^{2 N}\right)$ norm of their symbols, as illustrated in (3.35) and (3.36). The precise estimates of remainder terms in the Moyal expansion of the composition of two Weyl quantizations are presented here using Hermitian properties of the operator-valued exponential $e^{\frac{\mathrm{i}}{2 M^{1 / 2}}\left(\nabla_{x^{\prime}} \cdot \nabla_{p}-\nabla_{x} \cdot \nabla_{p^{\prime}}\right)}$. 
The Moyal expansions, see [18],

$$
\begin{gathered}
A(x) \# D(x, p)=e^{-\left.\frac{\mathrm{i}}{2 M^{1 / 2}} \nabla_{x^{\prime}} \cdot \nabla_{p^{\prime}} A\left(x+x^{\prime}\right) D\left(x, p+p^{\prime}\right)\right|_{x^{\prime}=p^{\prime}=0},} \\
C(x, p) \# D(x, p)=\left.e^{\frac{\mathrm{i}}{2 M^{1 / 2}}\left(\nabla_{x^{\prime}} \cdot \nabla_{p}-\nabla_{x} \cdot \nabla_{p^{\prime}}\right)} C(x, p) D\left(x^{\prime}, p^{\prime}\right)\right|_{(x, p)=\left(x^{\prime}, p^{\prime}\right)},
\end{gathered}
$$

are well defined for the symbols $A, C$ and $D$ in the Schwartz class, viewing the exponential as a Fourier multiplicator. We begin with the first expansion.

4.4.1. The Case $\boldsymbol{A}(\boldsymbol{x}) \# \boldsymbol{D}(\boldsymbol{x}, \boldsymbol{p})$. We study the remainder term for the expansion of the exponential using

$$
f_{x p}\left(x^{\prime}, p^{\prime}\right):=A\left(x+x^{\prime}\right) D\left(x, p+p^{\prime}\right)
$$

and apply the Fourier transform $\mathcal{F}$ defined for $f\left(x^{\prime}, p^{\prime}\right)$ by

$$
\mathcal{F}\{f\}\left(\xi_{x}, \xi_{p}\right):=\int_{\mathbb{R}^{2 N}} f\left(x^{\prime}, p^{\prime}\right) e^{-i\left(x^{\prime} \cdot \xi_{x}+p^{\prime} \cdot \xi_{p}\right)} \mathrm{d} x^{\prime} \mathrm{d} p^{\prime} .
$$

The remainder can be evaluated by the inverse Fourier transform

$$
\begin{aligned}
& \left.e^{-\frac{\mathrm{i}}{2 M^{1 / 2}} \nabla_{x^{\prime}} \cdot \nabla_{p^{\prime}}} f_{x p}\left(x^{\prime}, p^{\prime}\right)\right|_{x^{\prime}=p^{\prime}=0} \\
& \quad=\left(\frac{1}{2 \pi}\right)^{2 N} \int_{\mathbb{R}^{2 N}} \mathcal{F} f_{x p}\left(\xi_{x}, \xi_{p}\right) e^{\frac{\mathrm{i}}{2} M^{-1 / 2} \xi_{x} \cdot \xi_{p}} \mathrm{~d} \xi_{x} \mathrm{~d} \xi_{p}
\end{aligned}
$$

and Taylor expansion of the exponential function

$$
\begin{aligned}
&\left.e^{-\frac{\mathrm{i}}{2 M^{1 / 2}} \nabla_{x^{\prime}} \cdot \nabla_{p^{\prime}}} f_{x p}\left(x^{\prime}, p^{\prime}\right)\right|_{x^{\prime}=p^{\prime}=0} \\
&=\left(\frac{1}{2 \pi}\right)^{2 N} \int_{\mathbb{R}^{2 N}} \mathcal{F} f_{x p}\left(\xi_{x}, \xi_{p}\right) e^{\frac{\mathrm{i}}{2} M^{-1 / 2} \xi_{x} \cdot \xi_{p}} \mathrm{~d} \xi_{x} \mathrm{~d} \xi_{p} \\
&=\left(\frac{1}{2 \pi}\right)^{2 N} \int_{\mathbb{R}^{2 N}} \mathcal{F} f_{x p}\left(\xi_{x}, \xi_{p}\right)\left(\sum_{n=0}^{m}\left(\frac{\mathrm{i} \xi_{x} \cdot \xi_{p}}{2 M^{1 / 2}}\right)^{n} \frac{1}{n !}\right. \\
&\left.+\left(\frac{\mathrm{i} \xi_{x} \cdot \xi_{p}}{2 M^{1 / 2}}\right)^{m+1} \frac{1}{m !} \int_{0}^{1}(1-s)^{m} e^{\frac{\mathrm{i} s}{2} M^{-1 / 2} \xi_{x} \cdot \xi_{p}} \mathrm{~d} s\right) \mathrm{d} \xi_{x} \mathrm{~d} \xi_{p} \\
&=\left.\sum_{n=0}^{m} \frac{1}{n !}\left(-\frac{\mathrm{i} \nabla_{x^{\prime}} \cdot \nabla_{p^{\prime}}}{2 M^{1 / 2}}\right)^{n} f_{x p}\left(x^{\prime}, p^{\prime}\right)\right|_{x^{\prime}=p^{\prime}=0} \int_{0}^{1} e^{-\frac{\mathrm{i} s}{2} M^{-1 / 2} \nabla_{x^{\prime}} \cdot \nabla_{p^{\prime}}\left(-\mathrm{i} \nabla_{x^{\prime}} \cdot \nabla_{p^{\prime}}\right)^{m+1}} \\
&+\left.\left(\frac{1}{2 M^{1 / 2}}\right)^{m+1} \int_{0}^{m} \mathrm{~d} s\right|_{x^{\prime}=p^{\prime}=0} \cdot \\
& \times f_{x p}\left(x^{\prime}, p^{\prime}\right) \frac{(1-s)^{m}}{m !}
\end{aligned}
$$


The remainder is therefore

$$
\begin{aligned}
r(x, p)= & \left(\frac{1}{2 M^{1 / 2}}\right)^{m+1} \int_{0}^{1} e^{-\frac{\mathrm{i} s}{2} M^{-1 / 2} \nabla_{x^{\prime}} \cdot \nabla_{p^{\prime}}\left(-\mathrm{i} \nabla_{x^{\prime}} \cdot \nabla_{p^{\prime}}\right)^{m+1}} \\
& \times\left.\underbrace{f_{x p}\left(x^{\prime}, p^{\prime}\right)}_{A\left(x+x^{\prime}\right) B\left(x, p+p^{\prime}\right)} \frac{(1-s)^{m}}{m !} \mathrm{d} s\right|_{x^{\prime}=p^{\prime}=0} \\
= & \left(\frac{1}{2 M^{1 / 2}}\right)^{m+1} \int_{0}^{1} e^{-\frac{\mathrm{i} s}{2} M^{-1 / 2} \nabla_{x} \cdot \nabla_{p}}\left(-\mathrm{i} \nabla_{x} \cdot \nabla_{p}\right)^{m+1} \\
& \times\left. A(x) B\left(x^{\prime}, p\right) \frac{(1-s)^{m}}{m !} \mathrm{d} s\right|_{x^{\prime}=x} .
\end{aligned}
$$

4.4.2. The Case $C(x, p) \# D(x, p)$. As above we obtain

$$
\begin{aligned}
& C(x, p) \# D(x, p)=\left.e^{\frac{\mathrm{i}}{2 M^{1 / 2}}\left(\nabla_{x^{\prime}} \cdot \nabla_{p}-\nabla_{x} \cdot \nabla_{p^{\prime}}\right)} C(x, p) D\left(x^{\prime}, p^{\prime}\right)\right|_{(x, p)=\left(x^{\prime}, p^{\prime}\right)} \\
& =\left.\sum_{n=0}^{m} \frac{1}{n !}\left(\mathrm{i} \frac{\nabla_{x^{\prime}} \cdot \nabla_{p}-\nabla_{x} \cdot \nabla_{p^{\prime}}}{2 M^{1 / 2}}\right)^{n} C(x, p) D\left(x^{\prime}, p^{\prime}\right)\right|_{(x, p)=\left(x^{\prime}, p^{\prime}\right)} \\
& \quad+\left(\frac{\mathrm{i}}{2 M^{1 / 2}}\right)^{m+1} \int_{0}^{1} e^{\frac{\mathrm{i} s}{2} M^{-1 / 2}\left(\nabla_{x^{\prime}} \cdot \nabla_{p}-\nabla_{x} \cdot \nabla_{p^{\prime}}\right)}\left(\nabla_{x^{\prime}} \cdot \nabla_{p}-\nabla_{x} \cdot \nabla_{p^{\prime}}\right)^{m+1} \\
& \quad \times\left. C(x, p) D\left(x^{\prime}, p^{\prime}\right)\right|_{(x, p)=\left(x^{\prime}, p^{\prime}\right)} \frac{(1-s)^{m}}{m !} \mathrm{d} s .
\end{aligned}
$$

We can therefore write the remainder as

$$
\begin{aligned}
R(x, p) & \\
= & \left(\frac{\mathrm{i}}{2 M^{1 / 2}}\right)^{m+1} \int_{0}^{1} e^{\frac{\mathrm{i} s}{2} M^{-1 / 2}\left(\nabla_{x^{\prime}} \cdot \nabla_{p}-\nabla_{x} \cdot \nabla_{p^{\prime}}\right)}\left(\nabla_{x^{\prime}} \cdot \nabla_{p}-\nabla_{x} \cdot \nabla_{p^{\prime}}\right)^{m+1} \\
& \times\left. C(x, p) D\left(x^{\prime}, p^{\prime}\right)\right|_{(x, p)=\left(x^{\prime}, p^{\prime}\right)} \frac{(1-s)^{m}}{m !} \mathrm{d} s \\
= & \left.\int_{0}^{1} e^{\frac{\mathrm{i} s}{2} M^{-1 / 2}\left(\nabla_{x^{\prime}} \cdot \nabla_{p}-\nabla_{x} \cdot \nabla_{p^{\prime}}\right)} \tilde{R}\left(x, p, x^{\prime}, p^{\prime}, s\right)\right|_{x^{\prime}=x, p^{\prime}=p} \mathrm{~d} s,
\end{aligned}
$$

with

$$
\begin{aligned}
& \tilde{R}\left(x, p, x^{\prime}, p^{\prime}, s\right) \\
& \quad:=\left(\frac{\mathrm{i}}{2 M^{1 / 2}}\right)^{m+1}\left(\nabla_{x^{\prime}} \cdot \nabla_{p}-\nabla_{x} \cdot \nabla_{p^{\prime}}\right)^{m+1} C(x, p) D\left(x^{\prime}, p^{\prime}\right) \frac{(1-s)^{m}}{m !} \mathrm{d} s .
\end{aligned}
$$


Cauchy's inequality implies

$$
\begin{aligned}
& \|R(z)\|_{L^{2}\left(\mathbb{R}^{2 N}\right)}^{2} \\
& \quad=\int_{\mathbb{R}^{2 N}}\left|\int_{0}^{1} e^{\frac{\mathrm{i} s}{2} M^{-1 / 2}\left(\nabla_{x^{\prime}} \cdot \nabla_{p}-\nabla_{x} \cdot \nabla_{p^{\prime}}\right)} \tilde{R}\left(z, z^{\prime}, s\right) \mathrm{d} s\right|_{z=z^{\prime}}^{2} \mathrm{~d} z \\
& \quad \leq \int_{\mathbb{R}^{2 N}} \int_{0}^{1}\left|e^{\frac{\mathrm{i} s}{2} M^{-1 / 2}\left(\nabla_{x^{\prime}} \cdot \nabla_{p}-\nabla_{x} \cdot \nabla_{p^{\prime}}\right)} \tilde{R}\left(z, z^{\prime}, s\right)\right|_{z^{\prime}=z}^{2} \mathrm{~d} s \mathrm{~d} z \\
& \quad=\int_{0}^{1} \int_{\mathbb{R}^{2 N}}\left|e^{\frac{\mathrm{i} s}{2} M^{-1 / 2}\left(\nabla_{x^{\prime}} \cdot \nabla_{p}-\nabla_{x} \cdot \nabla_{p^{\prime}}\right)} \tilde{R}\left(z, z^{\prime}, s\right)\right|_{z^{\prime}=z}^{2} \mathrm{~d} z \mathrm{~d} s,
\end{aligned}
$$

which proves Lemma 3.11.

4.4.3. Estimates of $\|\boldsymbol{C} \# \boldsymbol{D}\|_{\boldsymbol{L}^{2}}$ and $\|\boldsymbol{A} \# \boldsymbol{D}\|_{\boldsymbol{L}^{2}}$. To verify (3.40), we insert in (4.31) the Fourier representation in the sense of distributions of the Dirac delta measure on $\mathbb{R}^{2 N}$,

$$
\delta\left(z-z^{\prime}\right)=(2 \pi)^{-2 N} \int_{\mathbb{R}^{2 N}} e^{\mathrm{i} \omega \cdot\left(z-z^{\prime}\right)} \mathrm{d} \omega,
$$

which implies

$$
\begin{aligned}
& e^{\frac{\mathrm{i} s}{2} M^{-1 / 2}\left(\nabla_{x^{\prime}} \cdot \nabla_{p}-\nabla_{x} \cdot \nabla_{p^{\prime}}\right)}\left(\tilde{R}\left(z, z^{\prime}, s\right) e^{\mathrm{i} \omega_{x} \cdot\left(x-x^{\prime}\right)+\mathrm{i} \omega_{p}\left(p-p^{\prime}\right)}\right) \\
& =e^{\mathrm{i} \omega_{x} \cdot\left(x-x^{\prime}\right)+\mathrm{i} \omega_{p}\left(p-p^{\prime}\right)} \\
& \times e^{\frac{\mathrm{i} s}{2} M^{-1 / 2}\left(\left(\nabla_{x^{\prime}}-\mathrm{i} \omega_{x}\right) \cdot\left(\nabla_{p}+\mathrm{i} \omega_{p}\right)-\left(\nabla_{x}+\mathrm{i} \omega_{x}\right) \cdot\left(\nabla_{p^{\prime}}-\mathrm{i} \omega_{p}\right)\right)} \tilde{R}\left(z, z^{\prime}, s\right) \\
& =e^{\mathrm{i} \omega_{x} \cdot\left(x-x^{\prime}\right)+\mathrm{i} \omega_{p}\left(p-p^{\prime}\right)} \\
& \times e^{\frac{\mathrm{i} s}{2} M^{-1 / 2}\left(\left(\nabla_{x^{\prime}} \cdot \nabla_{p}-\nabla_{x} \cdot \nabla_{p^{\prime}}\right)-\mathrm{i} \omega_{x} \cdot\left(\nabla_{p}+\nabla_{p^{\prime}}\right)+\mathrm{i} \omega_{p} \cdot\left(\nabla_{x}+\nabla_{x^{\prime}}\right)\right)} \tilde{R}\left(z, z^{\prime}, s\right),
\end{aligned}
$$

and

$$
\begin{aligned}
\int_{\mathbb{R}^{2 N}} & \left|e^{\frac{\mathrm{i} s}{2} M^{-1 / 2}\left(\nabla_{x^{\prime}} \cdot \nabla_{p}-\nabla_{x} \cdot \nabla_{p^{\prime}}\right)} \tilde{R}\left(z, z^{\prime}, s\right)\right|_{z=z^{\prime}}^{2} \mathrm{~d} z \\
= & \int_{\mathbb{R}^{4 N}}\left(e^{\frac{-\mathrm{i} s}{2} M^{-1 / 2}\left(\nabla_{x^{\prime}} \cdot \nabla_{p}-\nabla_{x} \cdot \nabla_{p^{\prime}}\right)} \tilde{R}^{*}\left(z, z^{\prime}, s\right)\right) \\
& \times\left(e^{\frac{\mathrm{i} s}{2} M^{-1 / 2}\left(\nabla_{x^{\prime}} \cdot \nabla_{p}-\nabla_{x} \cdot \nabla_{p^{\prime}}\right)} \tilde{R}\left(z, z^{\prime}, s\right)\right) \delta\left(z-z^{\prime}\right) \mathrm{d} z \mathrm{~d} z^{\prime} \\
= & \frac{1}{(2 \pi)^{2 N}} \int_{\mathbb{R}^{6 N}}\left(e^{\frac{-\mathrm{i} s}{2} M^{-1 / 2}\left(\nabla_{x^{\prime}} \cdot \nabla_{p}-\nabla_{x} \cdot \nabla_{p^{\prime}}\right)} \tilde{R}^{*}\left(z, z^{\prime}, s\right)\right) \\
& \times\left(e^{\frac{\mathrm{i} s}{2} M^{-1 / 2}\left(\nabla_{x^{\prime}} \cdot \nabla_{p}-\nabla_{x} \cdot \nabla_{p^{\prime}}\right)} \tilde{R}\left(z, z^{\prime}, s\right)\right) e^{\mathrm{i} \omega_{x} \cdot\left(x-x^{\prime}\right)} e^{\mathrm{i} \omega_{p} \cdot\left(p-p^{\prime}\right)} \mathrm{d} \omega_{x} \mathrm{~d} \omega_{p} \mathrm{~d} z \mathrm{~d} z^{\prime} .
\end{aligned}
$$


Integration by parts, property (4.32) and the fact that the differential operators $\nabla_{x^{\prime}} \cdot \nabla_{p}$ and $\nabla_{x} \cdot \nabla_{p^{\prime}}$ commute and are symmetric in $L^{2}\left(\mathbb{R}^{2 N}\right)$ show that

$$
\begin{aligned}
\int_{\mathbb{R}^{2 N}} & \left|e^{\frac{\mathrm{i} s}{2} M^{-1 / 2}\left(\nabla_{x^{\prime}} \cdot \nabla_{p}-\nabla_{x} \cdot \nabla_{p^{\prime}}\right)} \tilde{R}\left(z, z^{\prime}, s\right)\right|_{z=z^{\prime}}^{2} \mathrm{~d} z \\
= & \frac{1}{(2 \pi)^{2 N}} \int_{\mathbb{R}^{6 N}} \tilde{R}^{*}\left(z, z^{\prime}, s\right)\left(e^{\frac{-\mathrm{i} s}{2} M^{-1 / 2}\left(\nabla_{x^{\prime}} \cdot \nabla_{p}-\nabla_{x} \cdot \nabla_{p^{\prime}}\right)}\right. \\
& \left.\times\left(\left(e^{\frac{\mathrm{i} s}{2 \sqrt{M}}\left(\nabla_{x^{\prime}} \cdot \nabla_{p}-\nabla_{x} \cdot \nabla_{p^{\prime}}\right)} \tilde{R}\left(z, z^{\prime}, s\right)\right) e^{\mathrm{i} \omega_{x} \cdot\left(x-x^{\prime}\right)} e^{\mathrm{i} \omega_{p} \cdot\left(p-p^{\prime}\right)}\right)\right) \mathrm{d} \omega_{x} \mathrm{~d} \omega_{p} \mathrm{~d} z \mathrm{~d} z^{\prime} \\
= & \frac{1}{(2 \pi)^{2 N}} \int_{\mathbb{R}^{6 N}} \tilde{R}^{*}\left(z, z^{\prime}, s\right)\left(e^{\frac{-\mathrm{i} s}{2 \sqrt{M}}\left(\left(\nabla_{x^{\prime}} \cdot \nabla_{p}-\nabla_{x} \cdot \nabla_{p^{\prime}}\right)-\mathrm{i} \omega_{x}\left(\nabla_{p}+\nabla_{p^{\prime}}\right)+\mathrm{i} \omega_{p}\left(\nabla_{x}+\nabla_{x^{\prime}}\right)\right)}\right. \\
& \left.\times\left(e^{\frac{\mathrm{i} s}{2} M^{-1 / 2}\left(\nabla_{x^{\prime}} \cdot \nabla_{p}-\nabla_{x} \cdot \nabla_{p^{\prime}}\right)} \tilde{R}\left(z, z^{\prime}, s\right)\right)\right) \\
& \times e^{\mathrm{i} \omega_{x} \cdot\left(x-x^{\prime}\right)} e^{\mathrm{i} \omega_{p} \cdot\left(p-p^{\prime}\right)} \mathrm{d} \omega_{x} \mathrm{~d} \omega_{p} \mathrm{~d} z \mathrm{~d} z^{\prime} \\
= & \frac{1}{(2 \pi)^{2 N}} \int_{\mathbb{R}^{6 N}} \tilde{R}^{*}\left(z, z^{\prime}, s\right)\left(e^{\frac{-\mathrm{i} s}{2} M^{-1 / 2}\left(-\mathrm{i} \omega_{x}\left(\nabla_{p}+\nabla_{p^{\prime}}\right)+\mathrm{i} \omega_{p}\left(\nabla_{x}+\nabla_{x^{\prime}}\right)\right)} \tilde{R}\left(z, z^{\prime}, s\right)\right) \\
& \times e^{\mathrm{i} \omega_{x} \cdot\left(x-x^{\prime}\right)} e^{\mathrm{i} \omega_{p} \cdot\left(p-p^{\prime}\right)} \mathrm{d} \omega_{x} \mathrm{~d} \omega_{p} \mathrm{~d} z \mathrm{~d} z^{\prime} .
\end{aligned}
$$

Let

$$
\begin{aligned}
& u=\left(z+z^{\prime}\right) / 2, \\
& v=\left(z-z^{\prime}\right) / 2,
\end{aligned}
$$

then by (4.30) we can expand the derivative

$$
\left(\nabla_{x^{\prime}} \cdot \nabla_{p}-\nabla_{x} \cdot \nabla_{p^{\prime}}\right)^{m+1}
$$

in $\tilde{R}\left(z, z^{\prime}, s\right)$ and collect the derivatives with respect to $z$ and $z^{\prime}$ in the functions $\tilde{C}$ and $\tilde{D}$ as

$$
\tilde{R}\left(z, z^{\prime}, s\right)=\sum_{n=1}^{m+1} \tilde{C}_{n}(z) \tilde{D}_{n}\left(z^{\prime}\right)=\sum_{n=1}^{m+1} \tilde{C}_{n}(u+v) \tilde{D}_{n}(u-v)=: r(u, v) .
$$

The Fourier transform in the $u$-direction is the convolution

$$
\begin{aligned}
\hat{r}(\xi, v) & :=\sum_{n=1}^{m+1} \int_{\mathbb{R}^{2 N}} \tilde{C}_{n}(u+v) \tilde{D}_{n}(u-v) e^{-\mathrm{i} \xi \cdot u} \mathrm{~d} u \\
& =\frac{1}{(2 \pi)^{2 N}} \sum_{n=1}^{m+1} \int_{\mathbb{R}^{2 N}} e^{\mathrm{i} \zeta \cdot v} \mathcal{F} \tilde{C}_{n}(\zeta) e^{-\mathrm{i}(\xi-\zeta) \cdot v} \mathcal{F} \tilde{D}_{n}(\xi-\zeta) \mathrm{d} \zeta .
\end{aligned}
$$

The right-hand side in (4.33) becomes

$$
\begin{aligned}
& \frac{1}{(2 \pi)^{2 N}} \int_{\mathbb{R}^{6 N}} \tilde{R}^{*}\left(z, z^{\prime}, s\right)\left(e^{\frac{-\mathrm{i} s}{2} M^{-1 / 2}\left(-\mathrm{i} \omega_{x}\left(\nabla_{p}+\nabla_{p^{\prime}}\right)+\mathrm{i} \omega_{p}\left(\nabla_{x}+\nabla_{x^{\prime}}\right)\right)} \tilde{R}\left(z, z^{\prime}, s\right)\right) \\
& \quad \times e^{\mathrm{i} \omega_{x} \cdot\left(x-x^{\prime}\right)} e^{\mathrm{i} \omega_{p} \cdot\left(p-p^{\prime}\right)} \mathrm{d} \omega_{x} \mathrm{~d} \omega_{p} \mathrm{~d} z \mathrm{~d} z^{\prime} \\
& =\frac{1}{\pi^{2 N}} \int_{\mathbb{R}^{6 N}} r^{*}(u, v) e^{\frac{-\mathrm{i} s}{4} M^{-1 / 2}\left(-\mathrm{i} J \omega \cdot \nabla_{u}\right)+2 \mathrm{i} \omega \cdot v} r(u, v) \mathrm{d} \omega \mathrm{d} u \mathrm{~d} v
\end{aligned}
$$


which can be written

$$
\begin{aligned}
& \frac{1}{(2 \pi)^{2 N}} \int_{\mathbb{R}^{6 N}} \tilde{R}^{*}\left(z, z^{\prime}, s\right)\left(e^{\frac{-\mathrm{i} s}{2} M^{-1 / 2}\left(-\mathrm{i} \omega_{x}\left(\nabla_{p}+\nabla_{p^{\prime}}\right)+\mathrm{i} \omega_{p}\left(\nabla_{x}+\nabla_{x^{\prime}}\right)\right)} \tilde{R}\left(z, z^{\prime}, s\right)\right) \\
& \quad \times e^{\mathrm{i} \omega_{x} \cdot\left(x-x^{\prime}\right)} e^{\mathrm{i} \omega_{p} \cdot\left(p-p^{\prime}\right)} \mathrm{d} \omega_{x} \mathrm{~d} \omega_{p} \mathrm{~d} z \mathrm{~d} z^{\prime} \\
& =\frac{1}{\pi^{2 N}(2 \pi)^{2 N}} \int_{\mathbb{R}^{6 N}} \hat{r}^{*}(\xi, v) e^{\frac{-\mathrm{i} s}{4} M^{-1 / 2}(J \omega \cdot \xi)+2 \mathrm{i} \omega \cdot v} \hat{r}(\xi, v) \mathrm{d} \omega \mathrm{d} \xi \mathrm{d} v \\
& =\frac{1}{\pi^{2 N}} \int_{\mathbb{R}^{4 N}} \hat{r}^{*}(\xi, v) \hat{r}(\xi, v) \delta\left(2 v-\frac{s}{4} M^{-1 / 2} J \xi\right) \mathrm{d} \xi \mathrm{d} v \\
& =\frac{1}{(2 \pi)^{2 N}} \int_{\mathbb{R}^{2 N}}\left|\hat{r}\left(\xi, \frac{s}{8} M^{-1 / 2} J \xi\right)\right|^{2} \mathrm{~d} \xi,
\end{aligned}
$$

using definition (4.16) of the matrix $J$.

The next step is to determine

$$
\hat{r}(\xi, \gamma J \xi)=: \sum_{n=1}^{m+1} \hat{r}_{n}(\xi, \gamma J \xi)
$$

for $\gamma:=\frac{s}{8} M^{-1 / 2}$ by using the convolution

$$
\begin{aligned}
\left|\hat{r}_{n}(\xi, v)\right| & =\frac{1}{(2 \pi)^{2 N}}\left|\int_{\mathbb{R}^{2 N}} \mathcal{F} C_{n}(\zeta) e^{i \zeta \cdot v} \mathcal{F} D_{n}(\xi-\zeta) e^{-i(\xi-\zeta) \cdot v} \mathrm{~d} \zeta\right| \\
& \leq \frac{1}{(2 \pi)^{2 N}} \int_{\mathbb{R}^{2 N}}\left|\mathcal{F} C_{n}(\zeta)\right|\left|\mathcal{F} D_{n}(\xi-\zeta)\right| \mathrm{d} \zeta
\end{aligned}
$$

which by Young's inequality, namely $\|f * g\|_{L^{2}} \leq\|f\|_{L^{2}}\|g\|_{L^{1}}$, implies

$$
\begin{aligned}
& \frac{1}{(2 \pi)^{2 N}} \int_{\mathbb{R}^{2 N}}\left|\hat{r}_{n}\left(\xi, \frac{s}{8} M^{-1 / 2} J \xi\right)\right|^{2} \mathrm{~d} \xi \\
& =\frac{1}{(2 \pi)^{6 N}} \min \left(\left\|\mathcal{F} \tilde{C}_{n}\right\|_{L^{2}\left(\mathbb{R}^{2 N}\right)}^{2}\left\|\mathcal{F} \tilde{D}_{n}\right\|_{L^{1}\left(\mathbb{R}^{2 N}\right)}^{2},\left\|\mathcal{F} \tilde{C}_{n}\right\|_{L^{1}\left(\mathbb{R}^{2 N}\right)}^{2}\left\|\mathcal{F} \tilde{D}_{n}\right\|_{L^{2}\left(\mathbb{R}^{2 N}\right)}^{2}\right) \\
& =\frac{1}{(2 \pi)^{4 N}} \min \left(\left\|\tilde{C}_{n}\right\|_{L^{2}\left(\mathbb{R}^{2 N}\right)}^{2}\left\|\mathcal{F} \tilde{D}_{n}\right\|_{L^{1}\left(\mathbb{R}^{2 N}\right)}^{2},\left\|\mathcal{F} \tilde{C}_{n}\right\|_{L^{1}\left(\mathbb{R}^{2 N}\right)}^{2}\left\|\tilde{D}_{n}\right\|_{L^{2}\left(\mathbb{R}^{2 N}\right)}^{2}\right)
\end{aligned}
$$

that proves (3.40) in Lemma 3.12 and combined with (4.31), (4.33) and (4.34) it also establishes Lemma 3.11 .

Estimate (3.41) of $\|A(x) \# D(x, p)\|_{L^{2}\left(\mathbb{R}^{2 N}\right)}$ follows similarly from (4.29) by integration by parts

$$
\begin{aligned}
\int_{\mathbb{R}^{2 N}} & \left|e^{\frac{\mathrm{i}}{M^{1 / 2}} \nabla_{x} \cdot \nabla_{p}} A(x) D\left(x^{\prime}, p\right)\right|_{x^{\prime}=x}^{2} \mathrm{~d} x \mathrm{~d} p \\
= & \int_{\mathbb{R}^{3 N}}\left(e^{\frac{-\mathrm{i}}{M^{1 / 2}} \nabla_{x} \cdot \nabla_{p}} A^{*}(x) D^{*}\left(x^{\prime}, p\right)\right)\left(e^{\frac{\mathrm{i}}{M^{1 / 2}} \nabla_{x} \cdot \nabla_{p}} A(x) D\left(x^{\prime}, p\right)\right) \\
& \times \delta\left(x-x^{\prime}\right) \mathrm{d} x \mathrm{~d} x^{\prime} \mathrm{d} p \\
= & \frac{1}{(2 \pi)^{N}} \int_{\mathbb{R}^{4 N}} A^{*}(x) D^{*}\left(x^{\prime}, p\right) \\
& \times e^{\frac{-\mathrm{i}}{M^{1 / 2}} \nabla_{x} \cdot \nabla_{p}}\left(\left(e^{\frac{\mathrm{i}}{M^{1 / 2}} \nabla_{x} \cdot \nabla_{p}} A(x) D\left(x^{\prime}, p\right)\right) e^{\mathrm{i} \omega\left(x-x^{\prime}\right)}\right) \mathrm{d} x \mathrm{~d} x^{\prime} \mathrm{d} p \mathrm{~d} \omega
\end{aligned}
$$


and the Fourier transform, $\mathcal{F}_{p}$, in the $p$-direction

$$
\begin{aligned}
\int_{\mathbb{R}^{2 N}} & \left|e^{\frac{\mathrm{i}}{M^{1 / 2}} \nabla_{x} \cdot \nabla_{p}} A(x) D\left(x^{\prime}, p\right)\right|_{x^{\prime}=x}^{2} \mathrm{~d} x \mathrm{~d} p \\
= & \frac{1}{(2 \pi)^{N}} \int_{\mathbb{R}^{4 N}} A^{*}(x) D^{*}\left(x^{\prime}, p\right)\left(e^{\frac{-\mathrm{i}}{M^{1 / 2}}\left(\nabla_{x}+\mathrm{i} \omega\right) \cdot \nabla_{p}}\left(e^{\frac{\mathrm{i}}{M^{1 / 2}} \nabla_{x} \cdot \nabla_{p}} A(x) D\left(x^{\prime}, p\right)\right)\right) \\
& \times e^{\mathrm{i} \omega\left(x-x^{\prime}\right)} \mathrm{d} x \mathrm{~d} x^{\prime} \mathrm{d} p \mathrm{~d} \omega \\
= & \frac{1}{(2 \pi)^{2 N}} \int_{\mathbb{R}^{4 N}} A^{*}(x)\left(\mathcal{F}_{p} D\right)^{*}\left(x^{\prime}, \xi\right) e^{\frac{\mathrm{i}}{M^{1 / 2}} \omega \cdot \xi} A(x) \mathcal{F}_{p} D\left(x^{\prime}, \xi\right) \\
& \times e^{\mathrm{i} \omega\left(x-x^{\prime}\right)} \mathrm{d} x \mathrm{~d} x^{\prime} \mathrm{d} \xi \mathrm{d} \omega \\
= & \frac{1}{(2 \pi)^{N}} \int_{\mathbb{R}^{3 N}} A^{*}(x)\left(\mathcal{F}_{p} D\right)^{*}\left(x^{\prime}, \xi\right) A(x) \mathcal{F}_{p} D\left(x^{\prime}, \xi\right) \delta\left(x-x^{\prime}+\frac{\xi}{\sqrt{M}}\right) \mathrm{d} x \mathrm{~d} x^{\prime} \mathrm{d} \xi \\
= & \frac{1}{(2 \pi)^{N}} \int_{\mathbb{R}^{2 N}} A^{*}\left(x^{\prime}-\frac{\xi}{\sqrt{M}}\right)\left(\mathcal{F}_{p} D\right)^{*}\left(x^{\prime}, \xi\right) A\left(x^{\prime}-\frac{\xi}{\sqrt{M}}\right) \mathcal{F}_{p} D\left(x^{\prime}, \xi\right) \mathrm{d} x^{\prime} \mathrm{d} \xi \\
= & \frac{1}{(2 \pi)^{N}} \int_{\mathbb{R}^{2 N}}\left|A\left(x^{\prime}-\frac{\xi}{\sqrt{M}}\right)\right|^{2}\left|\mathcal{F}_{p} D\left(x^{\prime}, \xi\right)\right|^{2} \mathrm{~d} x^{\prime} \mathrm{d} \xi \\
\leq & \|A\|_{L^{\infty}\left(\mathbb{R}^{N}\right)}^{2}\|D\|_{L^{2}\left(\mathbb{R}^{2 N}\right) \cdot}^{2}
\end{aligned}
$$

The case $A(p) \# D(x, p)$ is analogous to $A(x) \# D(x, p)$.

In conclusion, these estimates bound the remainder symbols $r_{0}$ and $r_{2}$ used in Lemmas 3.8-3.10. Finally, using that Schwartz functions are dense in $L^{p}\left(\mathbb{R}^{2 N}\right)$, for any $p \geq 1$, we have proved Lemmas 3.11 and 3.12.

\section{Appendix A. Which Density Operator?}

If the density operators $e^{-\beta \hat{\bar{H}}}$ and $\widehat{e^{-\beta \bar{H}_{0}}}$ would differ only little, it would not matter which one we use as a reference. The proof of Theorem 3.6 shows that observables based on these two operators differ by the small amount of order $\mathcal{O}\left(M^{-1 / 2}\right)$ when the number of particles, $N$, is small compared to $M$. Since we do not know if this difference is small for larger number of particles, we may ask which density operator to use. The density operator $\hat{\rho}_{q}:=e^{-\beta \hat{H}}$ is a time-independent solution to the quantum Liouville--von Neumann equation

$$
\partial_{t} \hat{\rho}_{t}=\mathrm{i} M^{1 / 2}\left[\hat{\rho}_{t}, \hat{\bar{H}}\right]
$$

while the classical Gibbs density $e^{-\beta \bar{H}_{0}}$ is a time-independent solution to the classical Liouville equation

$$
\partial_{t} \rho_{t}=-\left\{\rho_{t}, \bar{H}_{0}\right\}
$$

The corresponding density matrix symbol $\rho_{q}$ is not a time-independent solution to the classical Liouville equation, since $0=\mathrm{i} M^{1 / 2}\left(\rho_{q} \# \bar{H}-\bar{H} \# \rho_{q}\right) \neq\left\{\rho_{q}, \bar{H}\right\}$, and the classical Gibbs density is not a time-independent solution to the quantum Liouville-von Neumann equation, since i $M^{1 / 2}\left(e^{-\beta \bar{H}_{0}} \# \bar{H}_{0}-\bar{H}_{0} \# e^{-\beta \bar{H}_{0}}\right) \neq$ $\left\{e^{-\beta \bar{H}_{0}}, \bar{H}_{0}\right\}=0$. We are lead to the question which Gibbs density to use and why use any Gibbs measure. This question is analyzed regarding the timedependence and the classical behavior in the following two subsections. 


\section{A.1. Why Should We Use the Gibbs Density?}

In Statistical Mechanics books, the Gibbs density is often derived as the marginal distribution of a subsystem weakly coupled to a heat bath, where the composite system is assumed to have the microcanonical distribution, see [4]. Here we give a variant of this derivation, assuming instead that the marginal distribution of the subsystem is determined by the subsystem Hamiltonian.

In molecular dynamics simulations one often wants to determine properties of a large macroscopic system with many particles, say $N \sim 10^{23}$. Such large particle systems cannot yet be simulated in a computer and one may then ask for a setting where a smaller system has similar properties as the large. Therefore, we seek an equilibrium density matrix that has the property that the marginal distribution for a subsystem has the same density as the whole system. We will below motivate how this assumption together with an assumption of weak coupling between the subsystem and the whole system leads to the Gibbs measure; in fact, it is enough to assume that the marginal distribution for the subsystem has an equilibrium density which is a function of the Hamiltonian for the subsystem.

Assume that the Hamiltonian symbol has been diagonalized and consider one component so that the Hamiltonians are scalar-valued, with coordinates $z_{s} \in \mathbb{R}^{2 n}$ and Hamiltonian $H_{s}\left(z_{s}\right)$ in the subsystem and coordinates $z_{b} \in$ $\mathbb{R}^{2(N-n)}$ and Hamiltonian $H_{b}\left(z_{b}, z_{s}\right)$ for a large heat bath environment system, i.e., $N \gg n$. The whole system has the Hamiltonian $H(z)=H_{s}\left(z_{s}\right)+H_{b}\left(z_{b}, z_{s}\right)$ with $z=\left(z_{s}, z_{b}\right) \in \mathbb{R}^{2 N}$. On the classical side, $F(H)$ for any differentiable function $F: \mathbb{R} \rightarrow(0, \infty)$ yields a non-normalized solution to the time- independent Liouville equation. We assume that

\section{Assumption A.1.}

$F: \mathbb{R} \rightarrow(0, \infty)$ is continuously differentiable,

$\int_{\mathbb{R}^{2 N}} F(H(z)) \mathrm{d} z$ and $\int_{\mathbb{R}^{2(N-n)}} F\left(H_{b}\left(z_{b}, z_{s}\right)\right) \mathrm{d} z_{b}$ are finite.

The non-normalized marginal distribution for the subsystem is then

$$
\int_{\mathbb{R}^{2(N-n)}} \frac{F\left(H_{b}\left(z_{b}, z_{s}\right)+H_{s}\left(z_{s}\right)\right)}{\frac{F\left(H_{b}\left(z_{b}, z_{s}\right)\right)}{\int_{\mathbb{R}^{2(N-n)} F\left(H_{b}\left(\bar{z}_{b}, z_{s}\right)\right) \mathrm{d} \bar{z}_{b}}}} \frac{F\left(H_{b}\left(z_{b}, z_{s}\right)\right)}{\int_{\mathbb{R}^{2(N-n)}} F\left(H_{b}\left(\bar{z}_{b}, z_{s}\right)\right) \mathrm{d} \bar{z}_{b}} \mathrm{~d} z_{b}
$$

which by the mean value theorem is equal to

$$
\frac{F\left(H_{b}\left(\tilde{z}_{b}, z_{s}\right)+H_{s}\left(z_{s}\right)\right)}{\frac{F\left(H_{b}\left(\tilde{z}_{b}, z_{s}\right)\right)}{\int_{\mathbb{R}^{2(N-n)}} F\left(H_{b}\left(\bar{z}_{b}, z_{s}\right)\right) \mathrm{d} \bar{z}_{b}}}
$$

for some $\tilde{z}_{b} \in \mathbb{R}^{2(N-n)}$, that depends on $H_{s}\left(z_{s}\right)$. We note that $\tilde{z}_{b}$ may be non-unique. For given $F$ and $H_{b}$, we introduce the notation

$$
\begin{aligned}
\tilde{z}_{b} & :=\tilde{z}_{b}\left(H_{s}\left(z_{s}\right)\right), \\
\tilde{H}_{b}\left(z_{s}, H_{s}\left(z_{s}\right)\right) & :=H_{b}\left(\tilde{z}_{b}\left(H_{s}\left(z_{s}\right)\right), z_{s}\right) .
\end{aligned}
$$

If the heat bath is uncoupled to the system, the value $\tilde{z}_{b}$ does not depend on $H_{s}$, i.e., $\tilde{H}_{b}\left(z_{s}, H_{s}\left(z_{s}\right)\right)=\tilde{H}_{b}\left(z_{s}, 0\right)$. We make two additional assumptions. 
Assumption A.2. The coupling between the heat bath and the system is weak in the sense that

$$
\tilde{H}_{b}\left(z_{s}, H_{s}\left(z_{s}\right)\right)=\tilde{H}_{b}\left(z_{s}, 0\right)+o\left(H_{s}\left(z_{s}\right)\right), \text { as } H_{s}\left(z_{s}\right) \rightarrow 0 .
$$

This assumption expresses that the coupling energy between the subsystem and the heat bath is much smaller than the subsystem energy $H_{s}\left(z_{s}\right)$. The second assumption is that the marginal distribution for the subsystem is a function of the subsystem Hamiltonian.

Assumption A.3. For any continuous $H_{b}$ there is a function $f$, a constant $C$ and a point $z_{s} \in \mathbb{R}^{2 n}$ such that

$$
f\left(H_{s}\left(z_{s}\right)\right)=C \frac{F\left(\tilde{H}_{b}\left(z_{s}, H_{s}\left(z_{s}\right)\right)+H_{s}\left(z_{s}\right)\right)}{F\left(\tilde{H}_{b}\left(z_{s}, H_{s}\left(z_{s}\right)\right)\right)}
$$

for a set of continuous subsystem Hamiltonians $H_{s}$, containing a sequence $H_{s}^{(n)}$ such that $H_{s}^{(n)}\left(z_{s}\right) \rightarrow 0$, not identically zero, with $\int_{\mathbb{R}^{2 n}} f\left(H_{s}^{(n)}\left(\bar{z}_{s}\right)\right) \mathrm{d} \bar{z}_{s}$ finite.

We obtain with the definitions

$$
\begin{aligned}
L(H) & :=\log F(H), \\
\ell(H) & :=\log f(H),
\end{aligned}
$$

and $\tilde{H}_{s}:=H_{s}\left(z_{s}\right)$ that

$$
\ell\left(\tilde{H}_{s}\right)=L\left(\tilde{H}_{b}\left(z_{s}, \tilde{H}_{s}\right)+\tilde{H}_{s}\right)-L\left(\tilde{H}_{b}\left(z_{s}, \tilde{H}_{s}\right)\right)+\log C,
$$

and note that the assumption that $F>0$ is differentiable shows that $L$ and $\ell$ also are differentiable. The continuity of $F$ and $L$ yields

$$
\log C=\ell(0),
$$

by choosing $\tilde{H}_{s}=0$, so that

$$
\begin{aligned}
\frac{\ell\left(\tilde{H}_{s}\right)-\ell(0)}{\tilde{H}_{s}} & =\frac{L\left(\tilde{H}_{b}\left(z_{s}, \tilde{H}_{s}\right)+\tilde{H}_{s}\right)-L\left(\tilde{H}_{b}\left(z_{s}, \tilde{H}_{s}\right)\right)}{\tilde{H}_{s}} \\
& =\int_{0}^{1} L^{\prime}\left(\tilde{H}_{b}\left(z_{s}, \tilde{H}_{s}\right)+t \tilde{H}_{s}\right) \mathrm{d} t
\end{aligned}
$$

which as $\tilde{H}_{s} \rightarrow 0$ combined with (A.3) and the differentiability of $\ell$ establishes

$$
\ell^{\prime}(0)=L^{\prime}\left(\tilde{H}_{b}\left(z_{s}, 0\right)\right), \quad \text { for all } \tilde{H}_{b}\left(z_{s}, 0\right) .
$$

Consequently, the function $L^{\prime}$ is constant, since $\tilde{H}_{b}\left(z_{s}, 0\right)=H_{b}\left(\tilde{z}_{b}(0), z_{s}\right)$ can take any value in $\mathbb{R}$ by varying $H_{b}$. Let the constant be $-1 / T$ so that

$$
L(H)=-\frac{H}{T}+\text { constant, for any } H \in \mathbb{R},
$$

and we have by definition (A.5) obtained the Gibbs density

$$
F(H)=e^{-\beta H},
$$

which by (A.4) shows that also the marginal is the Gibbs distribution 
Proposition A.4. Assumptions A.1, A.2 and A.3 imply

$$
f\left(H_{s}\right)=\frac{e^{-\beta H_{s}}}{\int_{\mathbb{R}^{2 n}} e^{-H_{s}\left(z_{s}\right)} \mathrm{d} z_{s}} .
$$

In conclusion, molecular dynamics simulations often seek the property that the classical equilibrium for the subsystem is the same as for the larger environment, since the subsystem is supposed to model a larger system. We have shown that the classical Gibbs density is the only differentiable function with this property, in the sense of the derivation based on Assumptions A.1, A.2 and A.3, while we do not know whether the symbol for the quantum density matrix $\rho_{q}$ has the same property for a large number of particles. Therefore, we prefer to use the Weyl-quantized classical Gibbs density $\widehat{e^{-\beta \bar{H}_{0}}}$ as our reference density, as in Theorems 3.7 and 3.2, since its drawback of being non-timeindependent solution to the quantum Liouville-von Neumann equation is mild, in the sense that the time-dependent perturbation is small for long time, as shown in Sect. A.2.

Remark A.5. Let us finally informally motivate why it seems difficult to find time-independent solutions to the quantum Liouville equation that are not functions of the Hamiltonian. An equilibrium density operator must commute with the Hamiltonian operator, by the Liouville--von Neumann equation, and consequently it is diagonalized by the same transformation as the Hamiltonian. The diagonalized density operator is then a function of the eigenvalues of the Hamiltonian operator if they are distinct, by mapping the eigenvalues of the Hamiltonian to the eigenvalues of the density operator. Assume that this mapping can be extended to a continuous mapping $F: \mathbb{R} \rightarrow \mathbb{R}_{+}$. We can then write the density operator as a function of the Hamiltonian, namely $\hat{\rho}=F(\hat{H})$.

\section{A.2. Time-Dependent Density Operators}

One criterion for a density operator is that it is a time- independent or approximately time-independent solution to the quantum Liouville--von Neumann equation, so that measurements of the observable at different times vary little. Here we show that the time-dependent perturbation of observables based on the initial density matrix $\widehat{e^{-\beta \bar{H}}}$, namely the quantization of the classical Gibbs density, is small up to time $t \ll M$, which in some sense justifies to use the density matrix $\widehat{e^{-\beta \bar{H}}}$. We include a motivation for an estimate which is uniform in the total number of particles $N$, based on a small system weakly coupled to a large heat bath.

Let $\hat{\rho}_{t}$ be the solution to the quantum Liouville-von Neumann equation (A.1), with initial data $\rho_{0}=e^{-\beta \bar{H}}$. Introduce the change of variables $\hat{\rho}_{t}=$ $\hat{\rho}_{0}+\hat{v}_{t}$, then the perturbation $v:[0, \infty) \times \mathbb{R}^{2 N} \rightarrow \mathbb{C}^{d \times d}$ satisfies

$$
\partial_{t} \hat{v}_{t}=\mathrm{i} M^{1 / 2}\left[\hat{v}_{t}, \hat{\bar{H}}\right]+\mathrm{i} M^{1 / 2}\left[\hat{\rho}_{0}, \hat{\bar{H}}\right], t>0, \quad \hat{v}_{0}=0 .
$$


As in (3.7), we see that $v$ is diagonal since $\rho_{0}$ is diagonal. By Duhamel's principle, we have

$$
\hat{v}_{t}=\mathrm{i} M^{1 / 2} \int_{0}^{t} e^{-\mathrm{i} M^{1 / 2} \sigma \hat{\bar{H}}}\left[\hat{\rho}_{0}, \hat{\bar{H}}\right] e^{\mathrm{i} M^{1 / 2} \sigma \hat{\bar{H}}} \mathrm{~d} \sigma .
$$

An observable based on this density matrix $\hat{\rho}_{t}$ can therefore be written

$$
\operatorname{Tr}\left(\hat{\bar{A}}_{\tau} \hat{\bar{B}}_{0} \hat{\rho}_{t}\right)=\operatorname{Tr}\left(\hat{\bar{A}}_{\tau} \hat{\bar{B}}_{0} \hat{\rho}_{0}\right)+\operatorname{Tr}\left(\hat{\bar{A}}_{\tau} \hat{\bar{B}}_{0} \hat{v}_{t}\right)
$$

where the time-dependent perturbation takes the form

$$
\begin{aligned}
& \operatorname{Tr}\left(\hat{\bar{A}}_{\tau} \hat{\bar{B}}_{0} \hat{v}_{t}\right)=\mathrm{i} M^{1 / 2} \int_{0}^{t} \operatorname{Tr}\left(\hat{\bar{A}}_{\tau} \hat{\bar{B}}_{0} e^{-\mathrm{i} M^{1 / 2} \sigma \hat{\bar{H}}}\left[\hat{\rho}_{0}, \hat{\bar{H}}\right] e^{\mathrm{i} M^{1 / 2} \sigma \hat{\bar{H}}}\right) \mathrm{d} \sigma \\
& =\mathrm{i} M^{1 / 2} \int_{0}^{t} \operatorname{Tr}\left(e^{\mathrm{i} M^{1 / 2} \sigma \hat{\bar{H}}} \hat{\bar{A}}_{\tau} e^{-\mathrm{i} M^{1 / 2} \sigma \hat{\bar{H}}} e^{\mathrm{i} M^{1 / 2} \sigma \hat{\bar{H}}} \hat{\bar{B}}_{0} e^{-\mathrm{i} M^{1 / 2} \sigma \hat{\bar{H}}}\left[\hat{\rho}_{0}, \hat{\bar{H}}\right]\right) \mathrm{d} \sigma \\
& =\mathrm{i} M^{1 / 2} \int_{0}^{t} \operatorname{Tr}\left(\hat{\bar{A}}_{\tau+\sigma} \hat{\bar{B}}_{\sigma}\left[\hat{\rho}_{0}, \hat{\bar{H}}\right]\right) \mathrm{d} \sigma .
\end{aligned}
$$

Let

$$
\hat{h}:=\left[\hat{\rho}_{0}, \hat{\bar{H}}\right]
$$

then the Moyal expansion for composition and $\left\{\bar{H}, e^{-\beta \bar{H}}\right\}=0$ imply

$$
\begin{aligned}
h & =e^{-\beta \bar{H}} \# \bar{H}-\bar{H} \# e^{-\beta \bar{H}} \\
& =e^{-\beta \bar{H}} \# \frac{|p|^{2}}{2} \mathrm{I}-\frac{|p|^{2}}{2} \mathrm{I} \# e^{-\beta \bar{H}}+e^{-\beta \bar{H}} \# \Lambda(x)-\Lambda(x) \# e^{-\beta \bar{H}} \\
& =2 \sum_{k=1}^{\infty} e^{-\beta \Lambda(x)} \frac{\mathrm{i}^{2 k+1}}{(2 k+1) !(4 M)^{(2 k+1) / 2}}\left(\nabla_{x} \cdot \nabla_{p}\right)^{2 k+1} e^{-\beta|p|^{2}} \Lambda(x),
\end{aligned}
$$

and we obtain by Lemma 3.11

$$
\begin{aligned}
h & =e^{-\beta \Lambda(x)} \frac{\mathrm{i}^{3}}{3(4 M)^{3 / 2}}\left(\nabla_{x} \cdot \nabla_{p}\right)^{3} e^{-\beta|p|^{2}} \Lambda(x)+\mathcal{O}\left(M^{-5 / 2}\right) \\
& =e^{-\beta \bar{H}(x)} \frac{\mathrm{i}^{3}}{3(4 M)^{3 / 2}}\left(\left(p \cdot \nabla_{x}\right)^{3} \Lambda(x)+3\left(p \cdot \nabla_{x}\right) \Delta \Lambda(x)\right)+\mathcal{O}\left(M^{-5 / 2}\right)
\end{aligned}
$$

which by Lemma 3.11 yields $\|h\|_{L^{2}\left(\mathbb{R}^{2 N}\right)}=\mathcal{O}\left(M^{-3 / 2}\right)$. The symbol $h$ is diagonal, since $\rho_{0}$ is diagonal. We conclude that on the timescale $t \ll M$ the time-dependent perturbation is small and we obtain

$$
\left|\frac{\operatorname{Tr}\left(\hat{\bar{A}}_{\tau} \hat{\bar{B}}_{0} \hat{v}_{t}\right)}{\operatorname{Tr}\left(\hat{\rho}_{0}\right)}\right|=\left|\mathrm{i} M^{1 / 2} \frac{\int_{0}^{t} \int_{\mathbb{R}^{2 N}} \operatorname{Tr}\left(\bar{A}_{\tau+\sigma} \# \bar{B}_{\sigma} h\right) \mathrm{d} z \mathrm{~d} \sigma}{\int_{\mathbb{R}^{2 N}} \operatorname{Tr} \rho_{0} \mathrm{~d} z}\right| \leq C \frac{t}{M} .
$$

The remainder $h$ includes error terms, as $\left(p \cdot \nabla_{x}\right)^{3} \Lambda$ and $(p \cdot \nabla) \Delta \Lambda$, that typically are large proportional to $N$. To obtain estimates that do not increase with $N$, we need a new setting: we consider as in Sect. A.1 a smaller system, with coordinates $\left(x_{s}, p_{s}\right)=z_{s} \in \mathbb{R}^{2 n}$, weakly coupled to a large heat bath, with coordinates $\left(x_{b}, p_{b}\right)=z_{b} \in \mathbb{R}^{2(N-n)}$, where $n \ll N$. We assume weak 
coupling, as in (A.3), which here means that $\Lambda\left(x_{s}, x_{b}\right)=\Lambda_{s}\left(x_{s}\right)+\Lambda_{b}\left(x_{s}, x_{b}\right)$ satisfies

$$
\left|\nabla_{x_{s}} \Lambda_{b}\left(x_{s}, x_{b}\right)\right| \ll 1
$$

Let $z=\left(z_{s}, z_{b}\right) \in \mathbb{R}^{2 N}$. To understand the heat bath perturbation with respect to the system Hamiltonian, $\bar{H}_{s}$, we can, on the operator level, study $e^{-\gamma \hat{\bar{H}}_{s}} e^{-\gamma \hat{\bar{H}}_{b}}=e^{-\gamma\left(\hat{\bar{H}}_{s}+\hat{\bar{H}}_{b}\right)-\gamma \hat{H}_{\delta}}$ based on the Baker-Campbell-Hausdorff expansion for $\hat{H}_{\delta}$, which to leading order satisfies

$$
\hat{H}_{\delta} \simeq \frac{1}{2}\left[\hat{\bar{H}}_{s}, \hat{\bar{H}}_{b}\right]=-\Delta_{x_{s}} \Lambda_{b}\left(x_{s}, x_{b}\right)-2 \nabla_{x_{s}} \Lambda_{b}\left(x_{s}, x_{b}\right) \cdot \nabla_{x_{s}} .
$$

This perturbation is small in the sense that it is bounded with respect to $N$, provided the coupling (A.6) is weak. Here $\gamma=\beta$ or $\gamma=\mathrm{i} M^{1 / 2} t$ for $\beta, t \in \mathbb{R}$. Does the remainder $\widehat{e^{-\beta \hat{\bar{H}}}}$ also give a perturbation that is bounded with respect to $N$ ? Integration by parts as follows in fact shows that the perturbation caused by $h$ is bounded with respect to $N$. Introduce the notation $D_{\sigma}:=$ $\bar{A}_{\tau+\sigma} \# \bar{B}_{\sigma}(z)$. We have by Lemma 3.11

$$
\begin{aligned}
\int_{\mathbb{R}^{2 N}} & \operatorname{Tr}\left(\bar{A}_{\tau+\sigma} \# \bar{B}_{\sigma} h\right) \mathrm{d} z \\
= & \frac{1}{16 M^{3 / 2}} \int_{0}^{1} \int_{\mathbb{R}^{2 N}} \operatorname{Tr}\left(D_{\sigma}\left(x^{\prime}, p\right) e^{\frac{\mathrm{i} r}{2 M^{1 / 2}} \nabla_{x} \cdot \nabla_{p}}\left(\nabla_{x} \cdot \nabla_{p}\right)^{3}\left(\Lambda(x) e^{-\beta|p|^{2} / 2}\right) \times\right. \\
& \left.\times e^{-\beta \Lambda\left(x^{\prime}\right)}\right)\left.\right|_{x=x^{\prime}} \mathrm{d} x \mathrm{~d} p(1-r)^{2} \mathrm{~d} r \\
= & \frac{1}{16 M^{3 / 2}} \int_{0}^{1} \int_{\mathbb{R}^{2 N}} \int_{\mathbb{R}^{2 N}} \operatorname{Tr}\left(e^{-\frac{\mathrm{i} r}{2 M^{1 / 2}} \nabla_{x} \cdot \nabla_{p}}\left(-\nabla_{x} \cdot \nabla_{p}\right)^{3}\left(D_{\sigma}\left(x^{\prime}, p\right) \Lambda(x)\right) \times\right. \\
& \left.\times e^{-\beta \Lambda\left(x^{\prime}\right)} e^{-\beta|p|^{2} / 2}\right)\left.\right|_{x=x^{\prime}} \mathrm{d} x \mathrm{~d} p(1-r)^{2} \mathrm{~d} r .
\end{aligned}
$$

We can write

$$
\left(\nabla_{x} \cdot \nabla_{p}\right)^{3}\left(D_{\sigma}\left(x^{\prime}, p\right) \Lambda(x)\right)=\sum_{|\alpha|=3} \partial_{p}^{\alpha} D_{\sigma}\left(x^{\prime}, p\right) \partial_{x}^{\alpha} \Lambda(x)
$$

and we assume that there is a constant $C$ such that

$$
\begin{aligned}
\left\|\partial_{x}^{\alpha} \Lambda\right\|_{L^{\infty}\left(\mathbb{R}^{N}\right)} & \leq C, \quad \text { for }|\alpha| \leq 3, \\
\sum_{|\alpha|=3}\left\|\partial_{p}^{\alpha} D_{\sigma}\right\|_{L^{2}\left(\mathbb{R}^{2 N}\right)} & \leq C,
\end{aligned}
$$

uniformly in $N$. The first assumption means that three derivatives of the eigenvalue are bounded, and the second assumption is based on the observable $D_{\sigma}$ defined by the initial observables $\bar{A}_{0}$ and $\bar{B}_{0}$. These two initial observables only depend on the system coordinates. The assumption of weak coupling is then related to the assumption for $D_{\sigma}$. The system dynamics $z_{s}\left(t, z_{0}\right) \in \mathbb{R}^{2 n}$, with initial data $z_{0}=\left(x_{s}, p_{s}, x_{b}, p_{b}\right)$, depends only weakly on the heat bath coordinate $x_{b}$ through $\Lambda_{b}$. A motivation for the last assumption in (A.7) is the following: to leading order we have

$$
D_{\sigma}\left(z_{0}\right) \simeq \bar{A}\left(0, z_{s}\left(\tau+\sigma, z_{0}\right)\right) \bar{B}\left(0, z_{s}\left(\sigma, z_{0}\right)\right)
$$


and the derivatives $\partial_{p}^{\alpha}$ of the right-hand side, with $z_{0}=(x, p)$, are determined by the flows $z^{\prime}, z^{\prime \prime}, z^{\prime \prime \prime}$ as in (4.20), based on assumption (4.21).

Lemma 3.12 implies that there is a constant $C$ such that the timedependent perturbation has the bound

$$
\begin{aligned}
& \left|\frac{\operatorname{Tr}\left(\hat{\bar{A}}_{\tau} \hat{\bar{B}}_{0} \hat{v}_{t}\right)}{\operatorname{Tr}\left(\hat{\rho}_{0}\right)}\right| \\
& \quad \leq \frac{\left\|e^{-\frac{\mathrm{i}}{2 M^{1 / 2}} \nabla_{x} \cdot \nabla_{p}}\left(-\nabla_{x} \cdot \nabla_{p}\right)^{3}\left(D_{\sigma}\left(x^{\prime}, p\right) \Lambda(x)\right)\right\|_{L^{2}\left(\mathbb{R}^{2 N}\right)}\left\|e^{-\beta \bar{H}}\right\|_{L^{2}\left(\mathbb{R}^{2 N}\right)}}{\frac{M}{t}\left\|e^{-\beta \bar{H}}\right\|_{L^{1}\left(\mathbb{R}^{2 N}\right)}} \\
& \quad \leq \frac{t\left\|e^{-\beta \bar{H}}\right\|_{L^{2}\left(\mathbb{R}^{2 N}\right)}}{M\left\|e^{-\beta \bar{H}}\right\|_{L^{1}\left(\mathbb{R}^{2 N}\right)}}\left\|\partial_{x}^{\alpha} \Lambda\right\|_{L^{\infty}\left(\mathbb{R}^{N}\right)} \sum_{|\alpha|=3}\left\|\partial_{p}^{\alpha} D_{\sigma}\right\|_{L^{2}\left(\mathbb{R}^{2 N}\right)} \\
& \quad \leq \frac{C}{M},
\end{aligned}
$$

which holds uniformly in $N$.

\section{Appendix B. Numerical Tests}

In this section, we present precise formulations of the numerical demonstrations in Sect. 2.3. We formulate a simple model problem in order to compare the Schrödinger density with the molecular dynamics density in (2.15) and (2.16). Then we present a similar model for comparing the Schrödinger position correlation observable with its molecular dynamics approximation. In this section, we describe the respective numerical methods that were used in numerical approximation of these quantities.

\section{B.1. Model Problem Formulation}

We define the model Hamiltonian $\hat{H}=-M^{-1} \mathrm{I} \Delta+V(x)$, where $V: \mathbb{R} \rightarrow \mathbb{R}^{2 \times 2}$ and $\mathrm{I}$ is the $2 \times 2$ identity matrix. We choose to construct the potential $V$ that yields an avoided crossing with a variable spectral gap depending on the parameter $\delta \in \mathbb{R}$. The construction is done in such a way that the smallest energy gap, $2 \delta$, appears at a single position, $x=0$. We define the matrix

$$
\bar{V}(x)=\left[\begin{array}{cc}
x+x^{2} & \delta \\
\delta & -x+x^{2}
\end{array}\right], \quad x \in \mathbb{R},
$$

with the eigenvalues

$$
\bar{\lambda}_{1}(x)=x^{2}-\sqrt{\delta^{2}+x^{2}}, \quad \bar{\lambda}_{2}(x)=x^{2}+\sqrt{\delta^{2}+x^{2}}
$$

and the normalized eigenvectors $\psi_{1}:=\bar{\psi}_{1} /\left\|\bar{\psi}_{1}\right\|_{2}, \psi_{2}:=\bar{\psi}_{2} /\left\|\bar{\psi}_{2}\right\|_{2}$ where

$$
\bar{\psi}_{1}=\left[\begin{array}{c}
\frac{x-\sqrt{\delta^{2}+x^{2}}}{\delta} \\
1
\end{array}\right], \quad \bar{\psi}_{2}=\left[\begin{array}{c}
\frac{x+\sqrt{\delta^{2}+x^{2}}}{\delta} \\
1
\end{array}\right] .
$$

The derivatives with respect to $x$ of the eigenvectors $\psi_{1}$ and $\psi_{2}$ are of order $1 / \delta$. We study how the size of the spatial derivative of the eigenvalues impacts the 
approximation of the observables. Therefore, we construct a family of matrices with the eigenvalues

$$
\lambda_{1}(x):=\bar{\lambda}_{1}(x)+a \cos (b x)-1, \quad \lambda_{2}(x):=\bar{\lambda}_{2}(x),
$$

illustrated in Fig. 3. Then we define the matrix-valued potential function $V(x)$

$$
V:=\Psi D \Psi^{*} \text {, where } D:=\left[\begin{array}{cc}
\lambda_{1} & 0 \\
0 & \lambda_{2}
\end{array}\right] \text {, and } \Psi:=\left[\begin{array}{l}
\psi_{1} \\
\psi_{2}
\end{array}\right] \text {. }
$$

\section{B.2. Numerical Approximation}

To solve the Schrödinger equation with the Hamiltonian $\hat{H}$, we discretize the computational domain $\Omega:=[-6,6]$ using the uniform mesh $x_{k}=-6+k \Delta x$, $k=0,1,2, \ldots, K, \Delta x=\frac{12}{K}$. The computational domain is chosen such that the quantum density $\mu_{\mathrm{qc}}(x),(2.15)$, approximately vanishes outside $\Omega$. In the presented numerical results, we use $K=751$. The eigenvalue problem $\hat{H} \Phi_{n}=$ $E_{n} \Phi_{n}$ is approximated using the second-order finite difference approximation of the Laplacian with the zero boundary conditions on the computational domain $\Omega$ resulting in the algebraic eigenvalue problem

$$
\boldsymbol{H}_{d} \phi_{n}=e_{n} \boldsymbol{\phi}_{n}
$$

with the matrix

$$
\begin{aligned}
& \boldsymbol{H}_{d}:=\frac{1}{2 M(\Delta x)^{2}}
\end{aligned}
$$

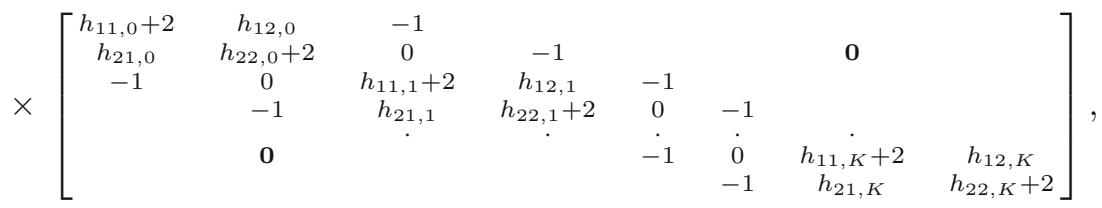

and approximation of the eigenfunctions

$$
\phi_{n}:=\left[\phi_{n, 0,1}, \phi_{n, 0,2}, \phi_{n, 1,1}, \phi_{n, 1,2}, \ldots, \phi_{n, K, 1}, \phi_{n, K, 2}\right]^{T},
$$

with the boundary condition $\phi_{n, 0, i}=\phi_{n, K, i}=0$ for $i=1,2$. The entries of the matrix are

$$
h_{i j, k}:=2 M(\Delta x)^{2} V_{i j}\left(x_{k}\right), \quad i, j=1,2 \quad k=0, \ldots, K .
$$

The algebraic eigenvalue problem has been solved using the Matlab function eig. In the numerical experiments reported here we used the parameters $a=1$ and $b=10$ in (B.3).

The quantum density $\mu_{\mathrm{qc}}(x),(2.15)$, is approximated by

$$
\frac{\sum_{n}\left(\left|\phi_{n, k, 1}\right|^{2}+\left|\phi_{n, k, 2}\right|^{2}\right) e^{-\beta e_{n}}}{\sum_{k} \sum_{n}\left(\left|\phi_{n, k, 1}\right|^{2}+\left|\phi_{n, k, 2}\right|^{2}\right) e^{-\beta e_{n} \Delta x}}, k=0,1, \ldots, N .
$$

Since the computational domain $\Omega$ is chosen large enough so that $\mu_{\mathrm{qc}}(x) \approx 0$ for $x \notin \Omega$, the imposed Dirichlet boundary condition does not introduce a significant numerical error. To approximate the integrals in the quotients of 
the molecular density $\mu_{\mathrm{cl}}(x)$, and the weight $q_{k},(2.16)$, we use the trapezoidal rule.

Correlation-Dependent Observables For numerical simulation of the position correlation observable, we deal with the expression $\operatorname{Tr}\left(\hat{x}_{\tau} \hat{x}_{0} e^{-\beta \hat{H}}\right)$ where $\hat{x}_{\tau}=$ $e^{\mathrm{i} \tau \sqrt{M} \hat{H}} \hat{x}_{0} e^{-\mathrm{i} \tau \sqrt{M} \hat{H}}$ for which we introduce the approximations

$$
e^{\mathrm{i} \tau \sqrt{M} \hat{H}} \simeq e^{\mathrm{i} \tau \sqrt{M} \boldsymbol{H}_{d}}, \quad e^{-\beta \hat{H}} \simeq e^{-\beta \boldsymbol{H}_{d}},
$$

and

$$
\hat{x}_{0} \simeq\left[\begin{array}{llllllll}
x_{0} & & & & & & \\
& x_{0} & x_{1} & & & & \\
& & & x_{1} & . & & \\
& & & & & \cdot & x_{K} & \\
0 & & & & & & & x_{K}
\end{array}\right]=: X .
$$

Next we define the matrices

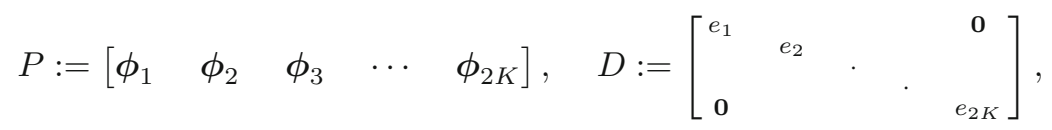

where the column vectors $\phi_{1}, \ldots, \phi_{K}$ and the numbers $e_{1}, \ldots, e_{K}$ solve the algebraic eigenvalue problem (B.4) with the mesh $x_{k}=-4.5+k \Delta x$, on the computational domain $[-4.5,4.5]$ for $\Delta x=\frac{9}{K}$ where $K=2048$. Note that the orthogonal matrix $P$ diagonalizes the real symmetric matrix $\boldsymbol{H}_{d}$, so $\boldsymbol{H}_{d}=$ $P D P^{T}$. Thus

$$
e^{\mathrm{i} \tau \sqrt{M} \boldsymbol{H}_{d}}=P e^{\mathrm{i} \tau \sqrt{M} D} P^{T}, \text { and } e^{-\beta \boldsymbol{H}_{d}}=P e^{-\beta D} P^{T} .
$$

We approximate the left-hand side of (2.17) by

$$
\frac{\operatorname{Tr}\left(P e^{\mathrm{i} \tau \sqrt{M} D} P^{T} X P e^{-\mathrm{i} \tau \sqrt{M} D} P^{T}\left(X P e^{-\beta D} P^{T}+P e^{-\beta D} P^{T} X\right)\right)}{\operatorname{Tr}\left(2 P e^{-\beta D} P^{T}\right)}
$$

and perform the calculations in Matlab.

For the right -hand side of the estimate (2.17), we solve the Hamiltonian dynamics (2.18) using a position Verlet scheme, see [11]. More specifically let $\left(x_{k}, p_{l}\right)=(k \Delta x, l \Delta p)$ be a partition of $[-4.5,4.5] \times[-4.5,4.5]$ where

$$
\Delta x=\frac{9}{K_{x}-1}, \Delta p=\frac{9}{K_{p}-1} \text { and } K_{x}=K_{p}=1000 .
$$

We compute for each $k, l \in\{-500,-499, \ldots, 499,500\}$ the path from the dynamics (2.18) and we approximate the integrals on the right-hand side of the estimate (2.17) with the two-dimensional trapezoidal method.

Choice of Parameters The model problem allows us to control the spectral gap by changing the parameter $\delta$ in the definition of $V(x)$. In the numerical simulations for the equilibrium densities, we have chosen $M=1000$. Note that in the atomic units used here the mass ratio for proton-electron system is approximately $M \approx 1836$. The temperature $T$ and the parameter $\delta$ were chosen such that the weight $q_{1}$ is kept the same and set to $q_{1}=0.8$. This choice guarantees that the contribution to the observable averaging from the excited state is not negligible. 
The simulation evaluating the error for the position correlation observable was done for the correlation time $\tau=0.2$ and the mass ratios up to $M=100$. Nonetheless, even this relatively small value of $M$ confirmed that the $L^{\infty}$-error is inversely proportional to the mass ratio $M$.

Open Access. This article is distributed under the terms of the Creative Commons Attribution 4.0 International License (http://creativecommons.org/licenses/ by/4.0/), which permits unrestricted use, distribution, and reproduction in any medium, provided you give appropriate credit to the original author(s) and the source, provide a link to the Creative Commons license, and indicate if changes were made.

\section{References}

[1] Alavi, A., Parrinello, M., Frenkel, D.: Ab initio calculation of the sound velocity of dense hydrogen: implications for models of Jupiter. Science 269(5228), 12521254 (1995)

[2] Bayer, C., Hoel, H., Kadir, A., Plecháč, P., Sandberg, M., Szepessy, A.: Computational error estimates for Born-Oppenheimer molecular dynamics with nearly crossing potential surfaces. Appl. Math. Res. Express 2, 329-417 (2015)

[3] Dall'ara, G.M.: Discreteness of the spectrum of Schrödinger operators with nonnegative matrix values potentials. J. Funct. Anal. 268(12), 3649-3679 (2015)

[4] Feynman, R.F.: Statistical Mechanics: A Set of Lectures. Westview Press, Boulder (1998)

[5] Folland, G.B.: Introduction to Partial Differential Equations. Princeton University Press, Princeton (1976)

[6] Folland, G.B.: Harmonic Analysis in Phase Space. Princeton University Press, Princeton (1989)

[7] Gronwall, T.H.: Note on the derivatives with respect to a parameter of the solutions of a system of differential equations. Ann. Math. 20(2), 292-296 (1919)

[8] Habershon, S., Manolopoulos, D.E., Markland, T.E., Miller 3rd, T.F.: Ringpolymer molecular dynamics: quantum effects in chemical dynamics from classical trajectories in an extended phase space. Annu. Rev. Phys. Chem. 64, 387-413 (2013)

[9] Kato, T.: Perturbation Theory for Linear Operators. Springer, Berlin (1980)

[10] LeBris, C.: Computational chemistry from the perspective of numerical analysis. Acta Numer. 14, 363-444 (2005)

[11] Leimkuhler, B., Matthews, C.: Molecular Dynamics. Springer, Berlin (2015)

[12] Marx, D., Hutter, J.: Ab Initio Molecular Dynamics: Basic Theory and Advanced Methods. Cambridge University Press, Cambridge (2009)

[13] Panati, G., Spohn, H., Teufel, S.: Space-adiabatic perturbation theory. Adv. Theor. Math. Phys. 7(1), 145-204 (2003)

[14] Panati, G., Spohn, H., Teufel, S.: The time-dependent Born-Oppenheimer approximation. ESAIM: M2AN 41(2), 297-314 (2007)

[15] Stiepan, H.-M., Teufel, S.: Semiclassical approximations for Hamiltonians with operator-valued symbols. Commun. Math. Phys. 320(3), 821-849 (2013) 
[16] von Neumann, J.: Thermodynamik quantenmechanischer Gesamtheiten. Nachr. Ges Wiss. Göttingen 3, 273-291 (1927)

[17] Wigner, E.: On the quantum correction for thermodynamic equilibrium. Phys. Rev. 40, 749-759 (1932)

[18] Zworski, M.: Semiclassical Analysis. American Mathematical Society, Providence (2012)

Aku Kammonen, Mattias Sandberg and Anders Szepessy

Institutionen för Matematik

Kungl. Tekniska Högskolan

10044 Stockholm

Sweden

e-mail: szepessy@kth.se

Aku Kammonen

e-mail: kammo@kth.se

Mattias Sandberg

e-mail: msandb@kth.se

Petr Plecháč

Department of Mathematical Sciences

University of Delaware

Newark DE 19716

USA

e-mail: plechac@udel.edu

Communicated by Claude Alain Pillet.

Received: September 21, 2017.

Accepted: May 11, 2018. 